Appl. Set-Valued Anal. Optim. 1 (2019), No. 3, pp. 231-305

Available online at http://asvao.biemdas.com

https://doi.org/10.23952/asvao.1.2019.3.04

\title{
VECTOR VARIATIONAL INEQUALITIES AND RELATED TOPICS: A SURVEY OF THEORY AND APPLICATIONS
}

\author{
NIKLAS HEBESTREIT \\ Department of Mathematics, Martin-Luther-University Halle-Wittenberg, Halle (Saale) 06120, Germany
}

\begin{abstract}
In this survey paper, we give a detailed introduction to some of the recent developments in the field of vector variational inequalities and related problems. By giving several examples and presenting the necessary mathematical background and theories, the survey attempts to draw a broad audience and is accessible to students in mathematics and engineering. In doing this, we will study several scalarization methods for vector variational inequalities, which are necessary, sufficient, or both, for the original problem. We further analyze topological and algebraical properties of the solution set. In particular, the existence of solutions is discussed by presenting several existence results. For this purpose, coercivity conditions ensuring, in a certain sense, the boundedness of the data of the vector variational inequality are required. We will further give a precise overview about existence results and techniques, which are known in the literature. Besides that, we analyze a regularization method for non-coercive vector variational inequalities, which consists of approximating solutions of non-coercive problems by a family of regularized vector variational inequalities. After that, we will study relations between vector variational inequalities and multi-objective optimization problems. Motivated by the duality principle in optimization, we also investigate two inverse vector variational inequalities. Furthermore, we consider gap functions for vector variational inequalities, which enable us to study equivalent optimization problems instead. A completely different approach consists of replacing the vector variational inequality by a parametric system or intersection problem. The idea of image space analysis is to study the vector problem in the image space, using one of the previous reformulations. Since vector variational inequalities are ill-posed in general, in the sense that they may either have no solution or multiple solutions, we study stability and sensitivity analysis results. Especially continuity properties of the corresponding solution mapping are investigated. Finally, we give a brief analysis of stochastic vector variational inequalities, generalized problems and numerical methods.
\end{abstract}

Keywords. Vector variational inequality; Inverse vector variational inequality; Perturbed vector variational inequality; Stochastic vector variational inequality; Vector quasi-variational inequality; Multiobjective optimization problem; Variational inequality; Linear and non-linear scalarization; Pareto reducibility; Coercivity condition; Compactness; Regularization; Gap function; Image space analysis; Stability; Ill-posed; Sensitivity; Numerical methods.

2010 Mathematics Subject Classification. 47J06, 49J40, 49K45, 49M29, 90C29, 90C31, 90C46.

E-mail address: niklas.hebestreit@mathematik.uni-halle.de.

Received September 18, 2019; Accepted October 30, 2019.

(C)2019 Applied Set-Valued Analysis and Optimization 


\section{CONTENTS}

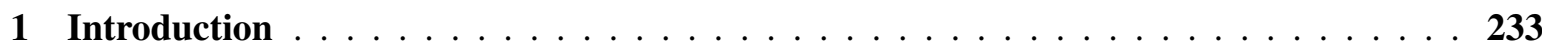

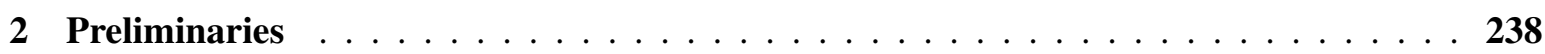

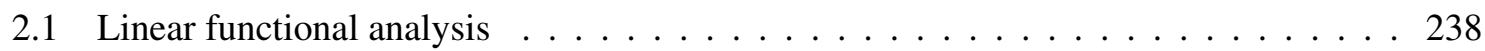

2.2 Functional analysis over cones . . . . . . . . . . . . . . . . . . 240

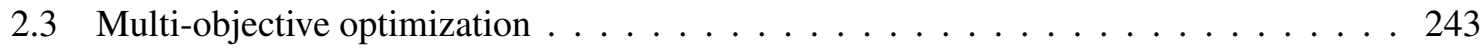

2.4 Vector variational inequalities . . . . . . . . . . . . . . . . . . . . 245

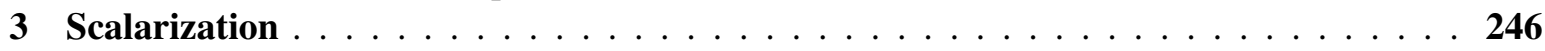

3.1 Linear scalarization . . . . . . . . . . . . . . . . . . . . 247

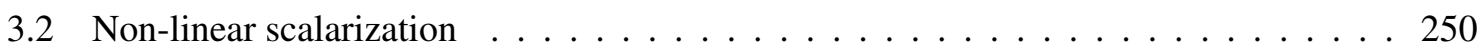

3.3 Pareto reducibility . . . . . . . . . . . . . . . . . . . 252

4 Topological and algebraic properties of the solution set $\ldots \ldots \ldots \ldots \ldots$

4.1 Boundedness, closedness and compactness . . . . . . . . . . . . . . . . . . . . . . . . . 256

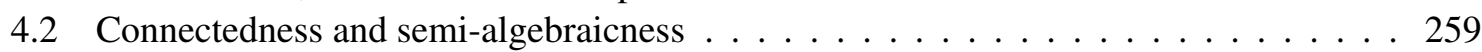

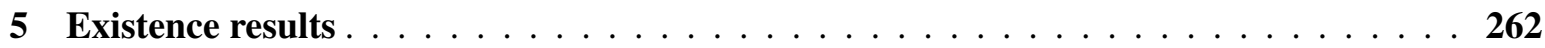

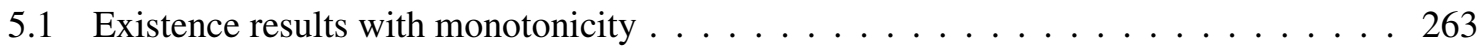

5.2 Existence results without monotonicity . . . . . . . . . . . . . . . . . 269

5.3 Regularization . . . . . . . . . . . . . . . . . . . . 275

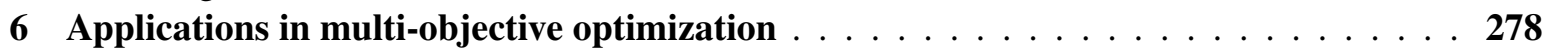

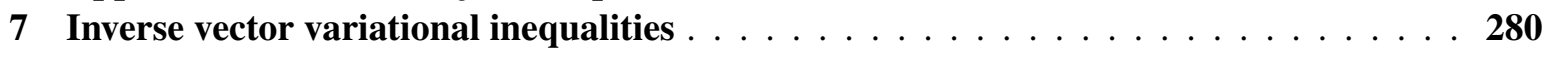

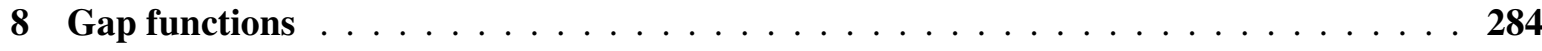

9 Image space analysis $\ldots \ldots \ldots \ldots \ldots \ldots \ldots \ldots \ldots$

9.1 Optimality conditions . . . . . . . . . . . . . . . . . . . . . . . 289

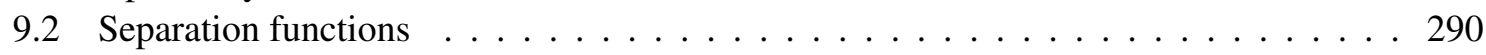

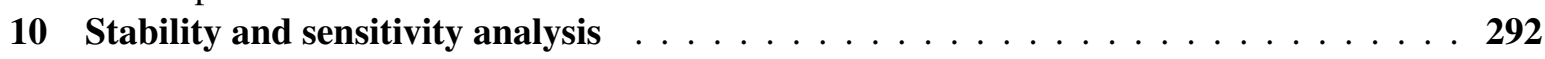

11 Further related topics . . . . . . . . . . . . . . . . . . . . 295

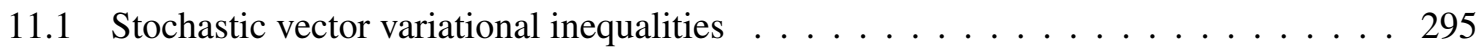

11.2 Generalized problems . . . . . . . . . . . . . . . . . . . . . 296

11.3 Numerical methods and algorithms . . . . . . . . . . . . . . . . . . . 297

References . . . . . . . . . . . . . . . . . . . . . . . . 297 


\section{INTRODUCTION}

In 1959, A. Signorini posed a contact problem in linear elasticity, which he called "problem with ambiguous boundary conditions". Some years later, in 1963, his student and friend G. Fichera gave a complete proof of the existence and uniqueness of a solution by formulating it as a variational inequality; see [91]. To honor his teacher, Fichera named the problem, proposed by Signorini, "Signorini problem". In addition to that, Fichera used in [91] the term "variational inequality" for the first time. One year later, the first cornerstone for the theory of variational inequalities was posed by G. Stampacchia [181] in order to study the regularity problem for partial differential equations, compare also [103, 144] for some of the main contributions of Stampacchia in the field of variational inequalities. Within the last years, variational problems have turned out to be a powerful tool in many fields of applied and industrial mathematics. Some prolific applications can be found, for example, in structural mechanics, physics, economics, optimization, and many other fields; see [69, 103, 113, 144, 171, 199] and the references therein. For this reason, the mathematical literature dedicated to variational inequalities is extensive, and the progress made in the past decades is impressive.

Later, in 1980, F. Giannessi [96] extended the notion of variational inequalities to the one of finitedimensional vector variational inequalities. He further provided some applications to alternative theorems, quadratic programms and complementary problems. Within the last 40 years, vector variational inequalities have turned out to be a powerful tool for studying numerous mathematical models in applied and industrial mathematics, for example, in multiobjective optimization and related fields which consist of the simultaneous investigation of contrary tasks; see [12, 73, 99, 108]. Compare also [200] for further references.

Motivating example. Let us investigate the following prominent problem: given a non-empty, closed and convex subset of $\mathbb{R}^{2}$ and a point $a^{1} \in \mathbb{R}^{2} \backslash C$, we are looking for the best approximation of $a^{1}$ (to the set $C$ w.r.t. the Euclidean norm $\|\cdot\|_{2}$ ), i.e. we consider the following optimization problem: find $x \in C$ with

$$
\left\|x-a^{1}\right\|_{2} \leq\left\|y-a^{1}\right\|_{2}, \quad \text { for every } \quad y \in C .
$$

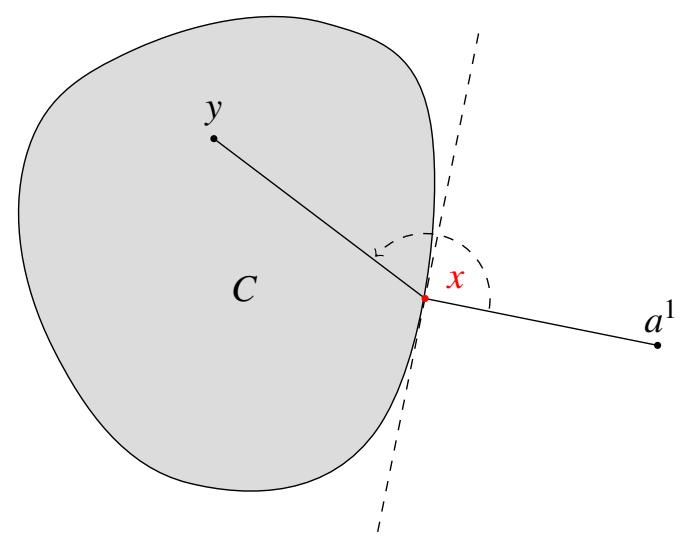

FIGURE 1. Geometric interpretation of a variational inequality

Figure 1 indicates that the best approximation of $a^{1}$ is characterized by the following observation: $x \in C$ is the best approximation of $a^{1}$ if and only if for all $y \in C$, the angle between $x-a^{1}$ and $x-y$ is greater than $90^{\circ}$, or equivalently,

$$
\measuredangle\left(x-a^{1}, x-y\right)=\frac{\left\langle x-a^{1}, x-y\right\rangle}{\left\|x-a^{1}\right\|_{2}\|x-y\|_{2}} \leq 0, \quad \text { for every } \quad y \in C .
$$


Therefore, finding the best approximation of $a^{1}$ is equivalent to solving the following variational inequality: find $x \in C$ such that

$$
\left\langle x-a^{1}, y-x\right\rangle \geq 0, \quad \text { for every } \quad y \in C .
$$

Let $a^{2} \in C$ be another point. We are now looking for all points $x \in C$ such that the Euclidean distance between $x-a^{1}$ and $x-a^{2}$ is minimal simultaneously. Here, minimization is understood in the sense that it is impossible to decrease the distance to $a^{1}$ (or $a^{2}$, respectively) without increasing the distance to $a^{2}$ (or $a^{1}$, respectively) at the same time. As illustrated in Figure 2, the element $\tilde{x}$ is non-minimal since one can shift it upwards which would cause a decrease of the distance between $\tilde{x}$ to the points $a^{1}$ and $a^{2}$ simultaneously. As we can see in the figure below, an element $x \in C$ is optimal if for every $y \in C$ the angles $x-a^{1}$ and $x-y$ as well as $x-a^{2}$ and $x-y$ are not bigger than $90^{\circ}$ at the same time, that is, either $\measuredangle\left(x-a^{1}, x-y\right) \leq 0$ or $\measuredangle\left(x-a^{2}, x-y\right) \leq 0$ for every $y \in C$. However, this is equivalent to saying that $x \in C$ is a solution of the following vector variational inequality: find $x \in C$ such that

$$
\left(\begin{array}{c}
\left\langle x-a^{1}, y-x\right\rangle \\
\left\langle x-a^{2}, y-x\right\rangle
\end{array}\right) \notin-\operatorname{int} \mathbb{R}_{\geq}^{2}, \quad \text { for every } \quad y \in C .
$$

It should be noted that the solution set of the vector variational inequality is given by the line segment $\left[a^{1}, a^{2}\right] \cap C$; compare Figure 2 .

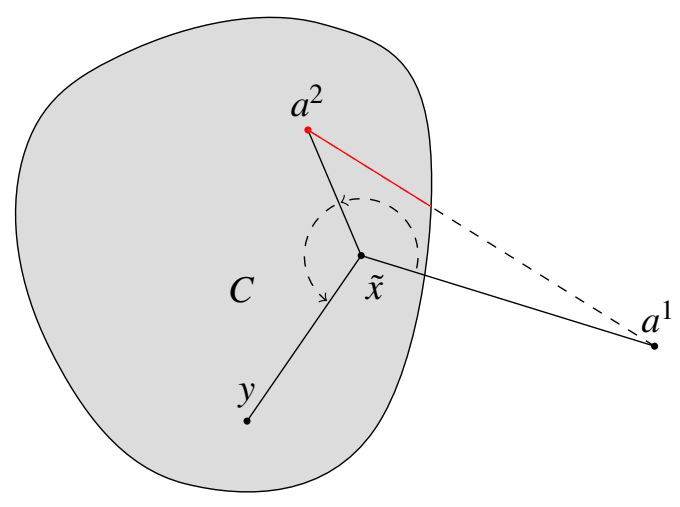

FIGURE 2. Geometric interpretation of a vector variational inequality

To be precise, let $X$ and $Y$ be real Banach spaces, let $C$ be a non-empty, closed and convex subset of $X$ and denote by $K$ a proper, closed, convex and solid cone in $Y$; compare Section 2 for basic notations and useful definitions, which we will use in this paper. Given a mapping $F: X \rightarrow \mathrm{L}(X, Y)$, which maps into the space of linear and bounded operators from $X$ to $Y$, the vector variational inequality, which will be studied in this survey, consists of finding an element $x \in C$ such that

$$
\langle F x, y-x\rangle_{Y} \notin-\operatorname{int} K, \quad \text { for every } \quad y \in C .
$$

In the above, $\langle F x, y-x\rangle_{Y}:=(F x)(y-x)$ denotes the evaluation of the mapping $F x$ at $y-x$. Clearly, if we let $X:=\mathbb{R}^{l}, Y:=\mathbb{R}^{k}$ and $K:=\mathbb{R}_{\geq}^{k}$, and use the identification $\mathrm{L}\left(\mathbb{R}^{l}, \mathbb{R}^{k}\right)=\operatorname{Mat}_{k \times l}(\mathbb{R})$, problem (1.1) recovers the following finite-dimensional vector variational inequality: find an element $x \in C$ such that

$$
\left(\begin{array}{c}
\left\langle F_{1} x, y-x\right\rangle \\
\vdots \\
\left\langle F_{k} x, y-x\right\rangle
\end{array}\right) \notin-\operatorname{int} \mathbb{R}_{\geq}^{k}, \quad \text { for every } \quad y \in C .
$$

Clearly, $F=\left(F_{1}, \ldots, F_{k}\right)^{\top}$ is a mapping from $\mathbb{R}^{l}$ to $\operatorname{Mat}_{k \times l}(\mathbb{R})$. Further, $\langle\cdot, \cdot\rangle$ denotes the scalar product in $\mathbb{R}^{l}$ and $\mathbb{R}_{\geq}^{k}$ denotes the Pareto cone in $\mathbb{R}^{k}$. However, by letting $Y:=\mathbb{R}$ and $K:=\mathbb{R}_{\geq}$in the vector 
variational inequality (1.1), where $\mathbb{R}_{>}$denotes the set of non-negative real numbers, the mapping $F$ becomes an operator from $X$ to $\mathrm{L}(X, \mathbb{R})=X^{*}$. Thus, problem (1.1) recovers the following variational inequality as special case: find an element $x \in C$ such that

$$
\langle F x, y-x\rangle_{X^{*}, X} \geq 0, \quad \text { for every } \quad y \in C .
$$

Here, $\langle\cdot, \cdot\rangle_{X^{*}, X}$ denotes the duality pairing between $X$ and $X^{*}$.

In Section 2 of this paper, we are going to recall very basic definitions and results from the field of convex analysis, linear and non-linear functional analysis and multi-objective optimization, which will be frequently used in this paper. We will further investigate some fundamental properties of the vector variational inequality (1.1); see Section 2.4.

Section 3 is devoted to the study of scalarization methods and related techniques for problem (1.1) and (1.2), respectively. By introducing a scalarization function

$$
\psi: Y \rightarrow \mathbb{R}
$$

one of the most powerful methods for vector variational inequalities is to study so-called scalar variational inequalities. In this setting, the variational inequality, depending on the scalarization function $\psi$, consists of finding an element $x=x(\psi) \in C$ such that

$$
\psi\left(\langle F x, y-x\rangle_{Y}\right) \geq 0, \quad \text { for every } \quad y \in C .
$$

Two types of scalarization functions have turned out to be very useful for vector variational inequalities:

1. Linear scalarization: if $\psi=s \in Y^{*}$ is a linear and continuous functional from $Y$ to $\mathbb{R}$, the resulting scalar variational inequality becomes: find an element $x=x(s) \in C$ such that

$$
\langle s \circ F x, y-x\rangle_{X^{*}, X} \geq 0, \quad \text { for every } \quad y \in C .
$$

It should be noted that $s \circ F$ maps from $X$ to $X^{*}$. If in addition, $s$ belongs to the dual cone of $K$, then problem (1.4) is necessary for the vector variational inequality (1.1).

2. Non-linear scalarization: for $\psi=\varphi^{K, e}$, where $\varphi^{K, e}$ denotes the Tammer-Weidner function, the resulting scalar variational inequality becomes: find an element $x \in C$ such that

$$
\varphi^{K, e}\left(\langle F x, y-x\rangle_{Y}\right) \geq 0, \quad \text { for every } \quad y \in C .
$$

In contrast to linear scalarization, it will turn out that problem (1.5) is equivalent to the vector variational inequality (1.1).

In Section 4, we are going to investigate several topological and algebraical properties of the solution set of problem (1.1) and (1.2), respectively. Some of them are compactness, convexity, connectedness and semi-algebraicness. These properties will be frequently used within the following sections.

In Section 5, we will be concerned with existence results for vector variational inequality (1.1). Within the last years, many researchers have proposed several existence results for problem (1.1) and generalizations. However, a careful study shows that only a handful of different techniques are used. In order to guarantee that vector variational inequality (1.1) has a solution, a so-called coercivity condition is needed, that is, the data of problem (1.1) have to be regular in a certain sense. Some frequently used coercivity conditions for vector variational inequality are the following:

1. The constraining set $C$ is bounded.

2. $F$ is $v$-coercive, that is, there exists a non-empty and compact subset $B$ of $X$ and an element $y_{0} \in B \cap C$ such that

$$
\left\langle F y, y_{0}-y\right\rangle_{Y} \in \operatorname{int} K, \quad \text { for every } \quad y \in C \backslash B .
$$


3. $C$ is unbounded and $F$ is weakly coercive, that is, there exists an element $x_{0} \in C$ and a functional $s$ in the quasi-interior of $K^{*}$ such that

$$
\lim _{\|x\|_{X \rightarrow+\infty}} \frac{\left\langle s \circ F x-s \circ F x_{0}, x-x_{0}\right\rangle_{X^{*}, X}}{\left\|x-x_{0}\right\|_{X}}=+\infty .
$$

Hence, in case that problem (1.1) is non-coercive, one cannot apply the existence results, which are known in the literature. For this purpose, we will study a regularization method instead. To be precise, let a mapping $R: X \rightarrow \mathrm{L}(X, Y)$ and a sequence $\left\{\varepsilon_{n}\right\} \subseteq \mathbb{R}_{>}$of non-negative parameters be given. Instead of problem (1.1), we are going to consider the following family of regularized vector variational inequalities: find an element $x_{n} \in C$ such that

$$
\left\langle F x_{n}+\varepsilon_{n} R x_{n}, y-x_{n}\right\rangle_{Y} \notin-\text { int } K, \quad \text { for every } \quad y \in C .
$$

Due to the nice features of $R$, which will be specified later, the mapping $F+\varepsilon_{n} R$ has significantly better properties than $F$ and every regularized problem (1.6) has a solution $x_{n}$. This allows us to study the sequence $\left\{x_{n}\right\}$ of regularized solutions, which, under some boundedness conditions, has a weakly or strongly convergent subsequence and any limit point is a solution of problem (1.1). By this, we can ensure the existence of solutions of non-coercive vector variational inequalities.

In Section 6, we are going to study applications of vector variational inequalities in the field of multiobjective optimization. To be precise, a multi-objective optimization problem consists of finding an element $x \in C$ such that

$$
\psi(y)-\psi(x) \notin-\operatorname{int} K, \quad \text { for every } \quad y \in C .
$$

However, if the objective mapping $\psi: X \rightarrow Y$ is $K$-convex and Gâteaux-differentiable with Gâteauxderivative $D_{\mathrm{G}} \psi: X \rightarrow \mathrm{L}(X, Y)$, then $x \in C$ solves problem (1.7) if and only if it is a solution of the vector variational inequality (1.1) with $F=D_{\mathrm{G}} \psi$.

Section 7 is devoted to the study of inverse vector variational inequalities for problem (1.1). This technique is closely related to the duality approach in (multi-objective) optimization and allows to embed problem (1.1) into two inverse vector variational inequalities. This approach enables us to give necessary and sufficient conditions. Denoting the generalized indicator mapping of the constraining set $C$ by $\chi_{C}$, that is, $\chi_{C}(x)=0$ for $x \in C$ and $\chi_{C}(x)=+_{Y}$ else, the first inverse vector variational inequality of problem (1.1) consists of finding an operator $U \in D\left(F^{-}\right)$such that

$$
\left\langle V-U,-F^{-} U\right\rangle_{Y} \aleph_{\text {int } K}^{1} \chi_{C}^{*}(U)-\chi_{C}^{*}(V), \quad \text { for every } \quad V \in \mathrm{L}(X, Y) \text { with } \chi_{C}^{*}(V) \neq \emptyset .
$$

In a similar way, the second inverse vector variational inequality reads as follows: find an operator $U \in D\left(F^{-}\right)$such that

$$
\left\langle V-U,-F^{-} U\right\rangle_{Y} \npreceq_{\text {int } K}^{2} \chi_{C}^{*}(U)-\chi_{C}^{*}(V), \quad \text { for every } \quad V \in \mathrm{L}(X, Y) .
$$

In the above, $F^{-}: \mathrm{L}(X, Y) \rightarrow X$ denotes the so-called adjoint mapping of $F$ while $\chi_{C}^{*}$ denotes the weak conjugate of $\chi_{C}$. We will show that, under suitable conditions, any solution of problem (1.9) generates one of the vector variational inequality (1.1), and any solution of problem (1.1) generates a solution of (1.8).

Section 8 deals with gap functions for vector variational inequality (1.1). By means of a singlevalued gap function $g: X \rightarrow \mathbb{R} \cup\{+\infty\}$ it is possible to convert problem (1.1) into an equivalent scalar optimization problem. In other words, an element $x \in C$ is a solution of the vector variational inequality (1.1) if and only if

$$
g(x)=\min _{y \in C} g(y) .
$$

Such a transformation is highly useful since it allows to apply powerful methods and algorithms from the field of optimization to find solutions of the vector variational inequality (1.1). Besides that, gap functions 
play a fundamental role for deriving error bounds. Such error bounds provide an upper estimate of the distance of an arbitrary feasible point to the solution set of the underlying problem, that is,

$$
\operatorname{dist}(x, \operatorname{Sol}(\mathrm{VVI})) \leq c \sqrt{g(x)}, \quad \text { for every } \quad x \in C,
$$

where Sol(VVI) denotes the solution set of problem (1.1) and $c>0$ is a constant.

Section 9 is devoted to the investigation of image space analysis for the following finite-dimensional vector variational inequality: find an element $x \in C$ such that

$$
\langle F x, f(y)-f(x)\rangle_{\mathbb{R}^{k}} \notin-\operatorname{int} \mathbb{R}_{\geq}^{k}, \quad \text { for every } \quad y \in C .
$$

In the above, $F: \mathbb{R}^{l} \rightarrow \operatorname{Mat}_{k \times l}(\mathbb{R})$ and $f: \mathbb{R}^{l} \rightarrow \mathbb{R}^{l}$ are given mappings. The non-empty constraining set $C$ is defined by $C:=\left\{x \in \mathbb{R}^{l} \mid g(x) \in \mathbb{R}_{\geq}^{m}\right\}$, where $g: \mathbb{R}^{l} \rightarrow \mathbb{R}^{m}$ is a mapping with $g\left[\mathbb{R}^{l}\right] \cap \mathbb{R}_{\geq}^{m} \neq \emptyset$. The basic idea of image space analysis is quite analogous to that, which led from Riemann integral to Lebesgue one: instead of performing the analysis in the space, where problem (1.10) is given, image space analysis does it in the space, where the images of the functions involved in problem (1.10) run. Evidently, an element $x \in C$ is a solution of problem (1.10) if and only if the following system in the unknown $y$ is impossible:

$$
u=\langle F x, f(x)-f(y)\rangle_{\mathbb{R}^{k}} \in \operatorname{int} \mathbb{R}_{\geq}^{k}, \quad v=g(y) \in \mathbb{R}_{\geq}^{m}, \quad y \in \mathbb{R}^{l} .
$$

The space $\mathbb{R}^{k} \times \mathbb{R}^{m}$, where the new variable $(u, v)$ runs, is called the image space associated with problem (1.10). By introducing the sets

$$
\mathscr{H}:=\operatorname{int} \mathbb{R}_{\geq}^{k} \times \mathbb{R}_{\geq}^{m} \text { and } \mathscr{K}(x):=\left\{(u, v) \in \mathbb{R}^{k} \times \mathbb{R}^{m} \mid u=\langle F x, f(x)-f(y)\rangle_{\mathbb{R}^{k}}, v=g(y), y \in \mathbb{R}^{l}\right\},
$$

we can claim that $x \in C$ is a solution of vector variational inequality (1.10) if and only if

$$
\mathscr{H} \cap \mathscr{K}(x)=\emptyset .
$$

To perform image space analysis, we can indifferently begin with system (1.11) or (1.12). The formulation (1.12) corresponds to adopt an algebraic and geometric language, which will lead us to use separation theorems, while (1.11) leads to adopt so-called theorems of the alternatives. By using (1.12), we introduce the following equivalent image problem: given $x \in \mathbb{R}^{l}$, find an element $(\tilde{u}, \tilde{v}) \in \mathscr{K}(x) \cap\left(\mathbb{R}^{k} \times \mathbb{R}_{\geq}^{m}\right)$ such that

$$
u \Varangle_{\text {int } \mathbb{R}_{\geq}^{k}} \tilde{u}, \quad \text { for every } \quad(u, v) \in \mathscr{H} \cap\left(\mathbb{R}^{k} \times \mathbb{R}_{\geq}^{m}\right) .
$$

As we can see, in the image space we define an optimization problem also when in the initial space we are dealing with a variational problem. This is because problem (1.10) is equivalent to the impossibility of the system (1.11). It should be further noted that image space analysis may be applied to any kind of problem, which can be expressed under the form of the inconsistency of a parametric system.

In Section 10, we present stability and sensitivity analysis results for a finite-dimensional vector variational inequality of type (1.2). It is well-known that variational problems are ill-posed in general, that is, they may either have no solution or multiple solutions, and, most importantly, small errors in data could lead to uncontrollable errors in its solution(s). Due to this, numerous authors considered problem (1.2) with respect to contaminated/perturbed data, depending on some parameters signifying the level of error in a certain sense. To be precise, let $U$ and $V$ be Banach spaces, the so-called parameter spaces, let $E: U \rightrightarrows \mathbb{R}^{l}$ be a set-valued mapping with non-empty, closed and convex values, and let $\mathscr{F}: V \times \mathbb{R}^{l} \rightarrow \operatorname{Mat}_{k \times l}(\mathbb{R})$ be a given mapping. Given parameters $\rho \in U$ and $\eta \in V$, the perturbed finite-dimensional vector variational inequality consists of finding an element $x=x(\rho, \eta) \in E(\rho)$ such that

$$
\langle\mathscr{F}(\eta, x), y-x\rangle_{\mathbb{R}^{k}} \notin-\operatorname{int} \mathbb{R}_{\geq}^{k}, \quad \text { for every } \quad y \in E(\rho) .
$$


The corresponding solution mapping associated to problem (1.13) is the set-valued mapping Sol : $U \times$ $V \rightrightarrows \mathbb{R}^{l}$, where for every $(\rho, \eta) \in U \times V, \operatorname{Sol}(\rho, \eta)$ denotes the solution set of problem (1.13). In the context of vector variational inequalities, numerous researchers have focused on continuity properties for the set-valued mapping Sol. Besides such stability analysis results, a second research focus is the sensitivity analysis of problem (1.13), that is, the quantitative analysis of the solution mapping. Such an analysis includes, for example, derivative results for the set-valued solution mapping Sol, which can be used to derive necessary optimality conditions for problem (1.13).

In Section 11, we give a brief review of stochastic vector variational inequalities, generalizations of problem (1.1) and numerical methods. Until now, mainly all of the research has been done in the deterministic setting without any uncertainty in the problem's data. In order to cover the cases where imperfect or unknown information is given, so-called finite-dimensional vector variational inequalities have been studied in the last years. To be precise, let $\mathscr{F}: \mathbb{R}^{l} \times \mathbb{R} \rightarrow$ Mat $_{k \times l}(\mathbb{R})$ be a given mapping and let $C$ be a non-empty, closed and convex subset of $\mathbb{R}^{l}$. Further, let $(\Omega, \mathscr{A}, \mathrm{P})$ be a probability space and let $\xi: \Omega \rightarrow \mathbb{R}$ be a random variable. Then, the finite-dimensional stochastic vector variational inequality consists of finding an element $x \in C$ such that almost surely

$$
\langle F(x, \xi(\omega)), y-x\rangle_{\mathbb{R}^{k}} \notin-\operatorname{int} \mathbb{R}_{\geq}^{k}, \quad \text { for every } \quad y \in C,
$$

or equivalently, $\mathrm{P}\left(\omega \in \Omega \mid\langle F(x, \xi(\omega)), y-x\rangle_{\mathbb{R}^{k}} \notin-\operatorname{int} \mathbb{R}_{\geq}^{k}\right.$, for every $\left.y \in C\right)=1$. In the second part of Section 11, we will consider two generalizations of problem (1.1). The first generalization is motivated by the concept of variable domination structure, which has turned out to be powerful and useful; especially in the field of multi-objective optimization. Roughly spoken, instead of comparing all arising element in problem (1.1) by using the fixed cone $K$ only, one compares elements using a set-valued mapping $\mathscr{K}: X \rightrightarrows Y$. $\mathscr{K}$ has the property that for all $x \in X$, the set $\mathscr{K}(x)$ is a proper, closed, convex and solid cone in $Y$. By this, the vector variational inequality with respect to the variable domination structure $\mathscr{K}$ consists of finding an element $x \in C$ such that

$$
\langle F x, y-x\rangle_{Y} \notin-\operatorname{int} \mathscr{K}(x), \quad \text { for every } \quad y \in C .
$$

Evidently, if $\mathscr{K}$ is constant, then problem (1.14) recovers (1.1). Besides that, as a second generalization, we will consider so-called vector quasi-variational inequalities. Roughly spoken, such problems arise from the vector variational inequality (1.1) by attaching it a set-valued fixed-point problem. To be precise, let $E: C \rightrightarrows E$ be a set-valued mapping with non-empty, closed and convex values. Then, the vector quasivariational inequality consists of finding an element $x \in C$ such that

$$
x \in E(x) \quad \text { and } \quad\langle F x, y-x\rangle_{Y} \notin-\operatorname{int} K, \quad \text { for every } y \in E(x) .
$$

Clearly, if $E$ is a constant mapping, say $E(x)=C$ for every $x \in C$, then problem (1.15) recovers the vector variational inequality (1.1).

\section{PRELIMINARIES}

In this section, we provide the mathematical background as it will be used in this paper. Throughout this paper, we denote by $\mathbb{N}=\{1,2,3, \ldots\}, \mathbb{R}, \mathbb{R}_{\geq}:=\{x \in \mathbb{R} \mid x \geq 0\}$ and $\mathbb{R}_{>}:=\{x \in \mathbb{R} \mid x>0\}$ the set of positive integers, real numbers, non-negative and positive real numbers, respectively.

2.1. Linear functional analysis. The purpose of this section is to provided basic results from linear functional analysis. We will assume that the reader is familiar with some elementary notions such as topological spaces, linear spaces, Banach spaces, and topological properties. The proof of the results, presented in this section, can be found, for example, in the standard textbooks [4, 90, 148, 180, 198, 199].

In what follows, unless otherwise indicated, $X$ and $Y$ denote real normed spaces with corresponding norms $\|\cdot\|_{X}$ and $\|\cdot\|_{Y}$, respectively. 
Definition 2.1 ([90, Chapter 6]). Let $A: X \rightarrow Y$ be an operator. $A$ is said to be

(i) linear if $A$ satisfies

$$
A(\alpha x+\beta y)=\alpha A x+\beta A y, \quad \text { for every } \quad x, y \in X, \alpha, \beta \in \mathbb{R} .
$$

(ii) continuous at the point $x \in X$ iff for each sequence $\left\{x_{n}\right\}$ in $X$,

$$
x_{n} \rightarrow x \text { implies } A x_{n} \rightarrow A x .
$$

$A$ is called continuous if it is continuous at each point $x \in X$.

(iii) bounded if there is a constant $c>0$ such that

$$
\|A x\|_{Y} \leq c\|x\|_{X}, \quad \text { for every } \quad x \in X .
$$

(iv) compact if $A$ is continuous, and $A$ maps bounded sets into relatively compact sets.

Proposition 2.1 ([90, Proposition 6.4]). A linear operator $A: X \rightarrow Y$ is continuous if and only if it is bounded.

Definition 2.2 ([90, Definition 6.5]). We denote the space of linear and continuous operators from $X$ to $Y$ by $\mathrm{L}(X, Y)$, that is,

$$
\mathrm{L}(X, Y):=\{A: X \rightarrow Y \mid A \text { is linear and bounded }\} .
$$

For $A \in \mathrm{L}(X, Y)$, the operator norm $\|A\|_{\mathrm{L}(X, Y)}$ is defined by

$$
\|A\|_{\mathrm{L}(X, Y)}:=\sup _{\|x\|_{X} \leq 1}\|A x\|_{Y} .
$$

Proposition 2.2 ([90, Proposition 6.6]). The operator norm $\|\cdot\|_{\mathrm{L}(X, Y)}$ is a norm. Let in addition $Y$ be a real Banach space. Then, $\mathrm{L}(X, Y)$ is a Banach space.

Definition 2.3 ([30, Definition 2.15]). A linear and continuous functional on $X$ is a linear and continuous operator

$$
f: X \rightarrow \mathbb{R}
$$

The set of all linear and continuous functionals on $X$ is called the dual space of $X$ and will be denoted by $X^{*}$, that is, we define $X^{*}:=\mathrm{L}(X, \mathbb{R})$. For the image of $x \in X$ under the functional $f$, we write

$$
\langle f, x\rangle_{X^{*}, X}:=f(x)
$$

and $\langle\cdot, \cdot\rangle_{X^{*}, X}$ is called the duality pairing between $X$ and $X^{*}$. We further define $X^{* *}:=\left(X^{*}\right)^{*}$, which is called the bidual space of $X$ and which consists of all linear continuous functionals from $X^{*}$ to $\mathbb{R}$.

Corollary 2.1. The dual space $X^{*}$ of any normed space $X$ is a Banach space.

Proposition 2.3 ([30, Proposition 2.20]). Let $X$ be a normed linear space. The operator $J: X \rightarrow X^{* *}$, defined by

$$
J(x)(f):=\langle f, x\rangle_{X^{*}, X}, \quad \text { for every } \quad x \in X, f \in X^{*},
$$

has the following properties:

(i) $J$ is linear and $\|J(x)\|_{X^{* *}}=\|x\|_{X}$ for all $x \in X$.

(ii) $J(X)$ is a closed subspace of $X^{* *}$ if and only if $X$ is a Banach space.

Definition 2.4 ([90, Definition 6.26]). The operator $J: X \rightarrow X^{* *}$, given in Proposition 2.3, is called the canonical embedding of $X$ and $X^{* *}$. We say that the normed space $X$ is reflexive if the canonical embedding $J: X \rightarrow X^{* *}$ is surjective.

Remark 2.1. Any finite-dimensional Banach space is reflexive; see [90, Proposition 6.27]. 
Theorem 2.1 (Riesz' Lemma, [30, Theorem 2.26]). Let X be a normed space. Then, the closed unit ball in $X$ is compact if and only if $X$ is finite-dimensional.

Definition 2.5 ([30, Definition 2.27]). Let $X$ be a Banach space. A sequence $\left\{x_{n}\right\}$ in $X$ is called weakly convergent in $X$ to an element $x \in X$ if

$$
\left\langle f, x_{n}\right\rangle_{X^{*}, X} \rightarrow\langle f, x\rangle_{X^{*}, X}, \quad \text { for every } f \in X^{*} .
$$

The weak convergence is denoted by $x_{n} \rightarrow x$.

Remark 2.2. Note that, in contrast to the weak convergence, we call the usual convergence with respect to the norm sometimes strong convergence. The following theorem provides a compactness result with respect to the weak topology introduced by the weak convergence.

Theorem 2.2 (Eberlein-Smulian Theorem, [198, Theorem 21.D]). Let $X$ be a reflexive Banach space. Then, each bounded sequence in $X$ has a weakly convergent subsequence.

Let us recall the following proposition, which will be used frequently. A proof can be found in [108, Lemma 3.9.1] and [198, Proposition 21.23].

Proposition 2.4 (Properties of weak and strong convergence). Suppose that $X$ and $Y$ are real Banach spaces and let $\left\{x_{n}\right\} \subseteq X,\left\{f_{n}\right\} \subseteq X^{*}$ and $\left\{T_{n}\right\} \subseteq \mathrm{L}(X, Y)$ be given sequences. Then it holds:

(i) The strong convergence $x_{n} \rightarrow x$ implies the weak convergence $x_{n} \rightarrow x$.

(ii) If $\operatorname{dim} X<+\infty$, then the weak convergence $x_{n} \rightarrow x$ implies the strong convergence $x_{n} \rightarrow x$.

(iii) If $x_{n} \rightarrow x$, then $\left\{x_{n}\right\}$ is bounded and

$$
\|x\|_{X} \leq \liminf _{n \rightarrow+\infty}\left\|x_{n}\right\|_{X}
$$

that is, $\|\cdot\|_{X}$ is weak sequentially lower semicontinuous.

(iv) It follows from $x_{n} \rightarrow x$ and $f_{n} \rightarrow f$ that

$$
\left\langle f_{n}, x_{n}\right\rangle_{X^{*}, X} \rightarrow\langle f, x\rangle_{X^{*}, X}
$$

(v) If $X$ is reflexive in addition, $f_{n} \rightarrow f$ and $x_{n} \rightarrow x$, then it follows that

$$
\left\langle f_{n}, x_{n}\right\rangle_{X^{*}, X} \rightarrow\langle f, x\rangle_{X^{*}, X}
$$

(vi) It follows from $x_{n} \rightarrow x$ and $T_{n} \rightarrow T$ that

$$
\left\langle T_{n}, x_{n}\right\rangle_{Y} \rightarrow\langle T, x\rangle_{Y}
$$

2.2. Functional analysis over cones. In this section, we recall some preliminary results from the field of functional analysis of cones. The proof of the results presented in this section can be found, for example, in the standard textbooks [12, 70, 108, 126, 129].

In what follows the topological interior, the closure and the boundary of a set $A$ in a topological space will be denoted by $\operatorname{int} A, \operatorname{cl} A$ and $\operatorname{bd} A$, respectively.

Definition 2.6 ([126, Definition 1.2]). Suppose that $Y$ is a real linear space and let $A$ and $B$ be non-empty subsets. Then, the Minkowski sum and Minowski difference of $A$ and $B$ will be denoted by

$$
A+B:=\{a+b \mid a \in A \text { and } b \in B\} \quad \text { and } \quad A-B:=\{a-b \mid a \in A \text { and } b \in B\},
$$

respectively, where the multiplication by a scalar $\lambda \in \mathbb{R}$ with $A$ is denoted by $\lambda A:=\{\lambda a \mid a \in A\}$. We further define $y+A:=A+y:=A+\{y\}$ for any $y \in Y$. For empty sets, we are going to use the conventions $A \pm \emptyset:=\emptyset \pm A:=\emptyset$ and $\lambda \emptyset:=\emptyset$, respectively.

In order to compare elements of abstract spaces, it is convenient to recall the notion of a cone and corresponding cone properties. 
Definition 2.7 ([129, Definition 2.1.9]). Let $Y$ be a real topological linear space. A non-empty set $K \subseteq Y$ is a cone if $\lambda K \subseteq K$ for every $\lambda \geq 0$. The cone $K$ is called

(i) convex if $K+K \subseteq K$,

(ii) proper (or non-trivial) if $K \neq\{0\}$ and $K \neq Y$,

(iii) closed if $C$ is a closed subset of $Y$,

(iv) pointed if $K \cap(-K)=\{0\}$,

(v) reproducing if $K-K=Y$,

(vi) solid if int $K \neq \emptyset$.

Remark 2.3. (i) Clearly, if $K$ is a cone, then $0 \in K$.

(ii) Obviously, the cone $K$ satisfies condition (i) if and only if $K$ is a convex set; see [126, Lemma 1.11]. The cone further satisfies $K \subseteq K+\{0\} \subseteq K+K$. Therefore, $K$ is convex if and only if $K+K=K$.

(iii) A typical example of a cone, which occurs in multi-objective optimization and in the field of vector variational inequalities, is in $\mathbb{R}^{k}$, the non-negative orthant or Pareto cone

$$
\mathbb{R}_{\geq}^{k}:=\left\{y \in \mathbb{R}^{k} \mid y_{j} \geq 0 \text { for } j=1, \ldots, k\right\} .
$$

(iv) Evidently, the cone $\mathbb{R}_{\geq}^{k}$ satisfies all the conditions of Definition 2.7.

Another important class of subsets in a real linear space is introduced in the following definition.

Definition 2.8 ([12, Definition 1.7], [126, Definition 1.10 (d)]). Let $Y$ be a real linear space, $A \subseteq Y$ a non-empty set and $K$ a proper cone in $Y$. A non-empty convex subset $B \subseteq Y$ is called a base for $K$, if each $y \in K \backslash\{0\}$ has a unique representation of the form $y=\lambda b$ for some $\lambda>0$ and $b \in B$. The cone

$$
\text { cone }(A):=\{y \in Y \mid y=\lambda a \text { for some } \lambda \geq 0 \text { and some } a \in A\}
$$

is called the cone generated by A.

Remark 2.4. (i) Note that, if $B$ is a convex base of a proper convex cone, then $0 \notin B$, because otherwise the representation of elements in $B$ is non-unique; see [12, Remark 1.9].

(ii) It is an important property of a base $B$ of a cone $K$ that cone $(B)=K$.

(iii) If $0 \in \operatorname{int} A$ for a non-empty subset $A$ of a real topological linear space $Y$, then cone $(A)=Y$. This implies in particular that it holds

$$
0 \notin \operatorname{int} K
$$

for every proper cone $K$ in $Y$. This fact will be used frequently in the next sections.

Definition 2.9 ([126, Definition 1.23]). Let $Y$ be a real linear space with a convex cone $K \subseteq Y$.

(i) The cone $K^{*}:=\left\{y^{*} \in Y^{*} \mid\left\langle y^{*}, y\right\rangle \geq 0\right.$ for every $\left.y \in K\right\}$ is called the dual cone for $K$.

(ii) The set qi $K^{*}:=\left\{y^{*} \in Y^{*} \mid\left\langle y^{*}, y\right\rangle>0\right.$ for every $\left.y \in K \backslash\{0\}\right\}$ is called the quasi-interior of the dual cone for $K$.

Remark 2.5. Note that $K^{*}$ is indeed a convex cone, that is, the previous definition makes sense. For $K=\{0\}$ and $K=Y$ one obtains $K^{*}=Y^{*}$ and $K^{*}=\{0\}$, respectively.

Example 2.1. It holds $\left(\mathbb{R}_{\geq}^{l}\right)^{*}=\mathbb{R}_{\geq}^{l}$ and $\mathrm{qi}\left(\mathbb{R}_{\geq}^{l}\right)^{*}=\operatorname{int}\left(\mathbb{R}_{\geq}^{l}\right)^{*}=\mathbb{R}_{>}^{l}$. Many non-trivial examples of cones can be found in $[12,126]$.

Lemma 2.1 ([126, Lemma 1.27, Corollary 3.19, Lemma 3.21]). Let $K$ be a convex cone in the real linear space $Y$. Then we have:

(i) If qi $K^{*}$ is non-empty, then $K$ is pointed.

(ii) If in addition $Y$ is a real locally convex space and $K$ has a base, then the quasi-interior qi $K^{*}$ of the dual cone for $K$ is non-empty. 
(iii) If $Y$ is locally convex and separated where the topology gives $Y$ as the topological dual space of $Y$ and $K$ is closed and solid, then we have int $K^{*}=$ qi $K^{*}$.

(iv) If $K$ is closed, then $K=\left\{y \in Y \mid\left\langle y^{*}, y\right\rangle \geq 0\right.$ for every $\left.y^{*} \in K^{*}\right\}$.

(v) If $K$ is solid, then it holds int $K=\left\{y \in Y \mid\left\langle y^{*}, y\right\rangle>0\right.$ for every $\left.y^{*} \in K^{*} \backslash\{0\}\right\}$.

The following result is known as Krein-Rutman theorem and can be found for example in [126, Theorem 3.38].

Theorem 2.3. In a real separable normed space $Y$ with a closed, convex and pointed cone $K$ the quasiinterior qi $K^{*}$ of the dual cone is non-empty.

Definition 2.10 ([12, Section 2.1]). Let $K$ be a convex and solid cone in the linear space $Y$ and let $a, b \in Y$ be given elements. We define binary relations in the following way:

$$
\begin{aligned}
a \leq_{K} b & : \Longleftrightarrow b-a \in K, \\
a \leq_{K} b & : \Longleftrightarrow b-a \notin K, \\
a \leq_{\text {int } K} b & : \Longleftrightarrow b-a \in \operatorname{int} K, \\
a \not_{\text {int } K} b & : \Longleftrightarrow b-a \notin \operatorname{int} K .
\end{aligned}
$$

The next proposition collects important properties of the binary relations, introduced in the previous definition.

Proposition 2.5 ([12, Proposition 2.5, Lemma 2.2, Lemma 2.3]). Let $K$ be a cone in the linear space $Y$. The binary relations defined in the previous definition have the following properties:

(i) The relation $\leq_{K}$ is a partial ordering, that is, $\leq_{K}$ is reflexive, transitive and antisymmetric, if and only if $K$ is a proper, convex and pointed cone.

(ii) The relation $\leq_{K}$ is compatible with scalar multiplication and addition, that is, for all $a, b, c \in Y$ and $\lambda \geq 0$, it holds that $a \leq_{K} b$ implies $\lambda a \leq_{K} \lambda b$ and $a \leq_{K} b$ implies $a+c \leq_{K} b+c$.

(iii) Let in addition $K$ be solid. The relation $\not_{\mathrm{int}} K$ is compatible with addition, that is, for all $a, b, c \in$ $Y$ and $\lambda \geq 0$, it holds that $a \mathbb{Z}_{\mathrm{int} K} b$ implies $a+c \not \mathbb{\mathrm { int }}_{K} b+c$.

(iv) If in addition $K$ is convex and solid, then for all $a, b, \in Y$ it holds that $a \leq_{K} b$ and $a \mathbb{Z}_{\operatorname{int} K} 0$ implies $b \not \operatorname{int} K 0$.

(v) If in addition $K$ is solid, then for all $a \in Y$ and $\lambda \geq 0$, it holds that $\lambda a \Varangle_{\text {int } K} 0$ implies $a \Varangle_{\text {int } K} 0$.

Definition 2.11 ([73, Section 1]). Let $K$ be a convex and solid cone in the linear space $Y$. For non-empty sets $A$ and $B$ in $Y$, we introduce the following weak binary set relations:

$$
\begin{aligned}
& A \preccurlyeq{ }_{\text {int } K}^{1} B \quad: \Longleftrightarrow \quad \exists a \in A, \forall b \in B: a \leq_{\text {int } K} b, \\
& A \aleph_{\text {int } K}^{1} B \quad: \Longleftrightarrow \quad \forall a \in A, \exists b \in B: a \not \operatorname{Lint}_{K} b, \\
& A \preccurlyeq_{\text {int } K}^{2} B \quad: \Longleftrightarrow \quad \forall a \in A, \exists b \in B: a \leq_{\text {int } K} b, \\
& A \aleph_{\text {int } K}^{2} B \quad: \Longleftrightarrow \quad \exists a \in A, \forall b \in B: a \not \operatorname{Lint}_{K} b \text {. }
\end{aligned}
$$

If $A=\{a\}$ or $B=\{b\}$ is a singleton, then we write $a \preccurlyeq_{\text {int } K}^{1} B$ and $A \preccurlyeq_{\text {int } K}^{1} b$ instead of $\{a\} \preccurlyeq_{\text {int } K}^{1} B$ and $A \preccurlyeq{ }_{\text {int } K}^{1}\{b\}$, respectively. The same convention holds for the other set relations. We further use the convention $A \aleph_{\text {int } K}^{2} \emptyset$ for every non-empty subset $A$ of $Y$.

Remark 2.6. (i) For non-empty subset $A$ and $B$ of $Y$, it holds $A \preccurlyeq_{\text {int } K}^{2} B$ if and only if $A \subseteq B-\operatorname{int} K$.

(ii) Relation $\preccurlyeq_{\text {int } K}^{2}$ is known in the literature as upper set less order relation, see [129]. 


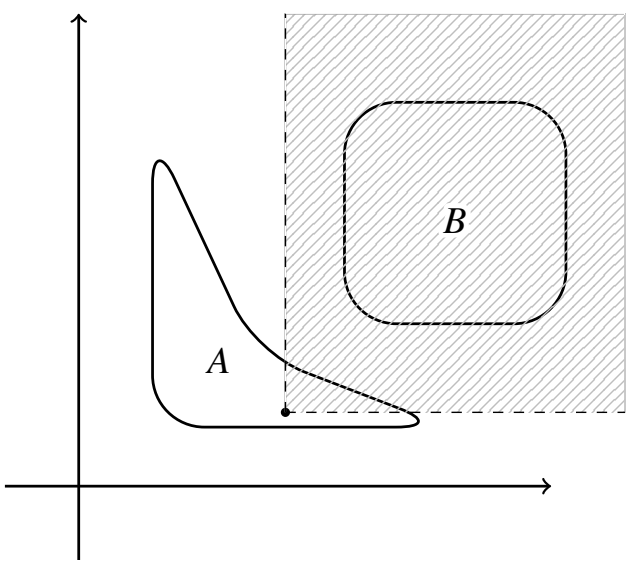

(A) $A \preccurlyeq{ }_{\text {int } \mathbb{R}_{\geq}^{2}}^{1} B$

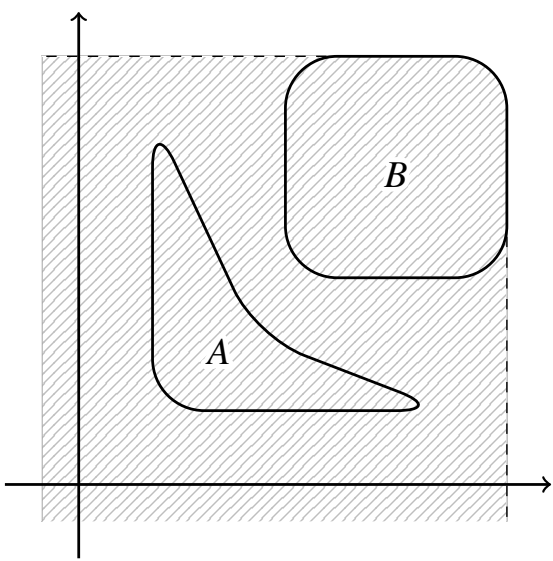

(C) $A \preccurlyeq_{\text {int } \mathbb{R}_{\geq}^{2}}^{2} B$

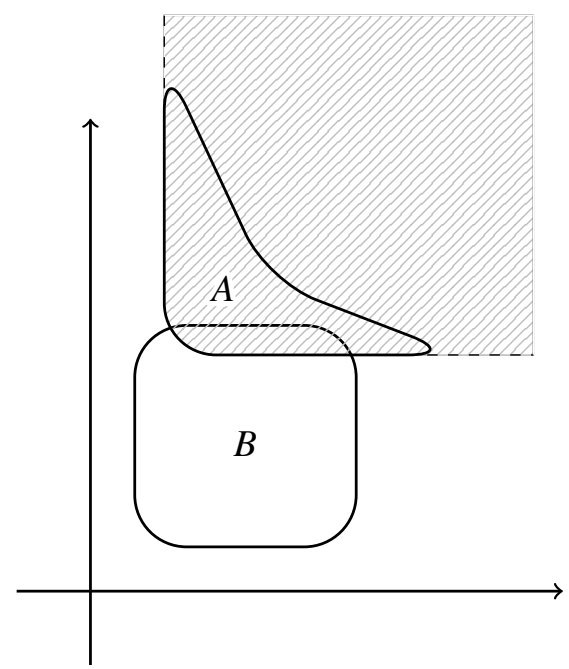

(B) $A \npreceq_{\text {int } \mathbb{R}_{\geq}^{2}}^{1} B$

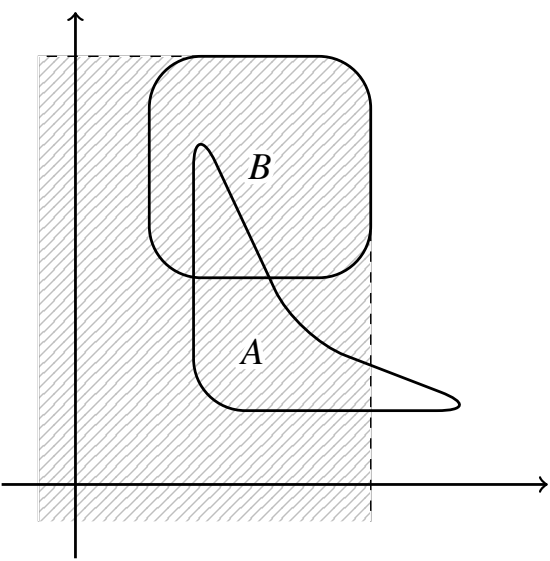

(D) $A \npreceq_{\text {int } \mathbb{R}_{>}^{2}}^{2} B$

FIGURE 3. Illustration of the set relations.

2.3. Multi-objective optimization. Many real-world problems require the minimization of different conflicting objectives; see, for example, $[70,72,126]$. Such problems can get formulated as multiobjective optimization problem (vector optimization problem), where the values of the objective mapping lie in a real topological linear space, which is ordered by a given cone.

The solution concept for multi-objective optimization problems is given in the next definition.

Definition 2.12 ([129, Section 2.4]). Let $X$ and $Y$ be real topological linear spaces, let $K$ be a proper and solid cone in $Y$ and let $\psi: X \rightarrow Y$ be a given mapping.

(i) An element $\psi(x) \in Y$, where $x \in X$, is said to be a weakly minimal element of $\psi$ if $\psi(y) \mathbb{E}_{\text {int } K}$ $\psi(x)$ for every $y \in Y$.

(ii) The set of weakly minimal elements of $\psi$ will be denoted by $\operatorname{WMin}(\psi[X], K)$.

(iii) Using the previous notation, the set

$$
\operatorname{WEff}(\psi[X], K):=\{x \in X \mid \psi(x) \in \operatorname{WMin}(\psi[X], K)\}
$$


is said to be the set of weakly efficient elements.

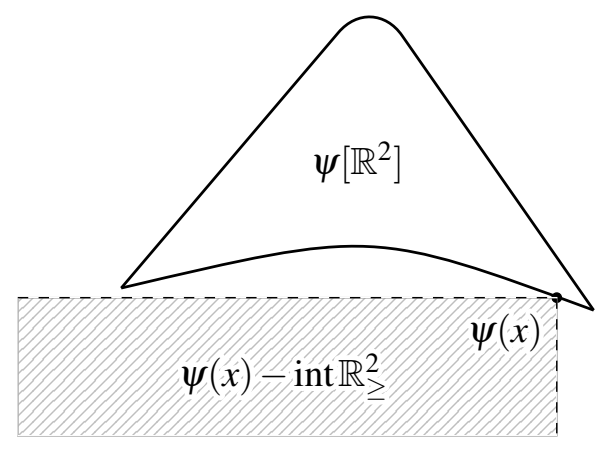

(A) $\psi(x)$ is a weakly minimal element of $\psi$

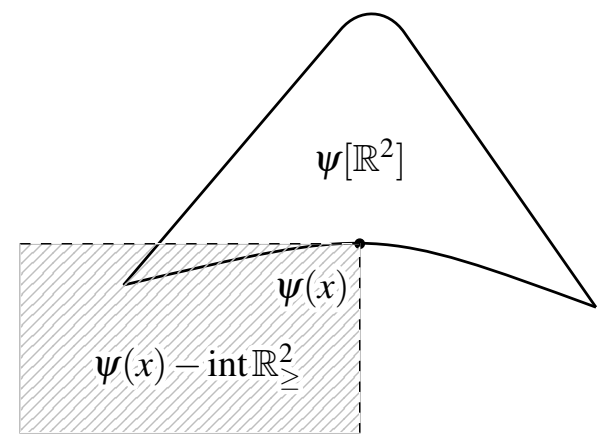

(B) $\psi(x)$ is not a weakly minimal element of $\psi$

FIGURE 4. Illustration of the solution concept.

Definition 2.13 ([129, Section 2.4]). Let $X$ and $Y$ be real topological linear spaces, let $K$ be a proper and solid cone in $Y$ and let $\psi: X \rightarrow Y$ be a given mapping.

(i) An element $\psi(x) \in Y$, where $x \in X$, is said to be a weakly maximal element of $\psi$ if $\psi(x) \mathbb{Z}_{\text {int } K}$ $\psi(y)$ for every $y \in Y$.

(ii) The set of weakly minimal elements of $\psi$ will be denoted by $\operatorname{WMax}(\psi[X], K)$.

In the following, we recall a prominent non-linear scalarizing function, which has been used frequently in the field of multi-objective optimization.

Definition 2.14 ([108, Section 2.3]). Let $Y$ be a real topological space, let $K$ be a proper, closed, convex and solid cone in $Y$ and let $e \in \operatorname{int} K$. The function

$$
\varphi^{K, e}: Y \rightarrow \mathbb{R} \cup\{ \pm \infty\}, \quad \varphi^{K, e}(y):=\inf \{t \in \mathbb{R} \mid y \in t e-K\}
$$

for every $y \in Y$, is called Tammer-Weidner function.

Theorem 2.4 ([108, Corollary 2.3.5]). Let $Y$ be a real topological space, let $K$ be a proper, closed, convex and solid cone in $Y$ and let $e \in \operatorname{int} K$. Then, the Tammer-Weidner function $\varphi^{K, e}$ is well-defined, finite-valued, continuous, sublinear and for every $\lambda \in \mathbb{R}$,

$$
\left\{y \in Y \mid \varphi^{K, e}(y) \leq \lambda\right\}=\lambda e-K, \quad\left\{y \in Y \mid \varphi^{K, e}(y)<\lambda\right\}=\lambda e-\operatorname{int} K
$$

Moreover, $\varphi^{K, e}$ is strictly int $K$-monotone. 


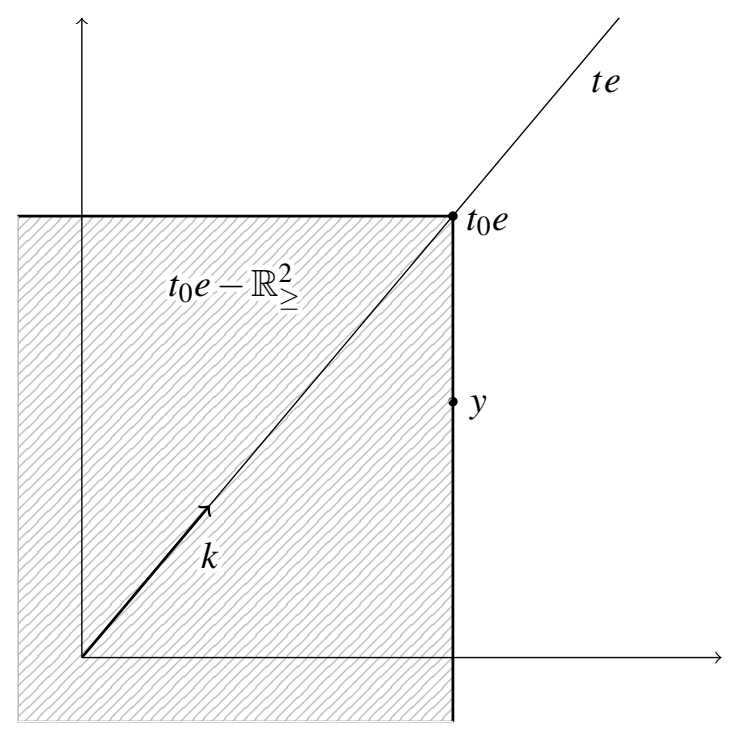

FIGURE 5. Visualization of the Tammer-Weidner function.

2.4. Vector variational inequalities. The purpose of this section is to introduce the notion of vector variational inequalities. We will restrict our attention to one of the simplest formulations, which can be found in the literature, because one can extend nearly all notions and results to generalized problems. To be precise, let $X$ and $Y$ be real Banach spaces, let $C$ be a non-empty, closed and convex subset of $X$, and let $K$ be a proper, closed, convex and solid cone in $Y$. If $F: X \rightarrow \mathrm{L}(X, Y)$ is a given mapping, which maps into the space $\mathrm{L}(X, Y)$ of linear and bounded operators from $X$ and $Y$, then the vector variational inequality consists of finding an element $x \in C$ such that

$$
\langle F x, y-x\rangle_{Y} \notin-\operatorname{int} K, \quad \text { for every } \quad y \in C .
$$

The set $C$ is frequently called constraining set. It should be noted that, in order to ensure that (2.1) is well-defined, one has to assume that the cone is proper and solid. If $X$ and $Y$ are finite-dimensional spaces, say $X=\mathbb{R}^{l}$ and $Y=\mathbb{R}^{k}$, and $K=\mathbb{R}_{\geq}^{k}$ is the Pareto cone in $\mathbb{R}^{k}$, then problem (2.1) becomes the following finite-dimensional vector variational inequality: find an element $x \in C$ such that

$$
\left(\begin{array}{c}
\left\langle F_{1} x, y-x\right\rangle \\
\vdots \\
\left\langle F_{k} x, y-x\right\rangle
\end{array}\right) \notin-\operatorname{int} \mathbb{R}_{\geq}^{k}, \quad \text { for every } \quad y \in C .
$$

Here, $F=\left(F_{1}, \ldots, F_{k}\right)^{\top}$ is a mapping from $\mathbb{R}^{l}$ to $\operatorname{Mat}_{k \times l}(\mathbb{R})$. In the above, $\langle\cdot, \cdot\rangle$ denotes the scalar product in $\mathbb{R}^{l}$. From the representation of int $\mathbb{R}_{\geq}^{k}$ follows that, if there exists at least one index $j \in\{1, \ldots, k\}$ such that $x \in C$ satisfies the variational inequality

$$
\left\langle F_{j} x, y-x\right\rangle \geq 0, \quad \text { for every } \quad y \in C,
$$

then $x \in C$ is necessary a solution of problem (2.2). Further, the solution set of problem (2.1) and (2.2) will be denoted by Sol(VVI) and Sol(VVI'), respectively.

In what follows, we will use the following assumptions, which we abbreviate to (A):

(A1) $X$ is a real reflexive Banach space. $Y$ is a real Banach space.

(A2) The constraining set $C$ is a non-empty, closed and convex subset of $X$.

(A3) $K$ is a proper, closed, convex, and solid cone in $Y$. 
Remark 2.7. Problem (2.1) is frequently called monotone vector variational inequality if the mapping $F$ is monotone in a certain sense; see [195, Definition 4]. Similar, a polynomial vector variational inequality is a vector variational inequality of the type (2.1), where $F$ is a polynomial.

The following example will serve as prototype example in this paper.

Example 2.2. Let $a^{1}, \ldots, a^{k}$ be different elements in $\mathbb{R}^{k}$. We consider the following finite-dimensional vector variational inequality: find an element $x \in \mathbb{R}^{l}$ such that

$$
\left(\begin{array}{c}
\left\langle x-a^{1}, y-x\right\rangle \\
\vdots \\
\left\langle x-a^{k}, y-x\right\rangle
\end{array}\right) \notin-\operatorname{int} \mathbb{R}_{\geq}^{k}, \quad \text { for every } \quad y \in \mathbb{R}^{l} .
$$

In the above, the constraining set $C$ is equal to $\mathbb{R}^{l}$, while the objective mapping $F: \mathbb{R}^{l} \rightarrow$ Mat $_{k \times l}(\mathbb{R})$ is given by

$$
F x:=\left(\begin{array}{c}
x-a^{1} \\
\vdots \\
x-a^{k}
\end{array}\right), \quad \text { for every } \quad x \in \mathbb{R}^{l} .
$$

We denote the solution set of problem (2.3) by S. Let $j \in\{1, \ldots, k\}$ be arbitrarily chosen and consider the variational inequality of finding an element $x \in \mathbb{R}^{l}$ such that

$$
\left\langle x-a^{j}, y-x\right\rangle \geq 0, \quad \text { for every } \quad y \in \mathbb{R}^{l} .
$$

Let us show that $a^{j}$ is the unique solution of the above variational inequality. Clearly, by inserting $a^{j}$, we see that $a^{j}$ solves the variational inequality. Now, assume to the contrary that there is a second solution $\tilde{a}^{j}$. This is impossible, since this would imply

$$
\left\|\tilde{a}^{j}-a^{j}\right\|_{2}=\left\langle\tilde{a}^{j}-a^{j}, a^{j}-\tilde{a}^{j}\right\rangle \leq 0
$$

and consequently, $a^{j}=\tilde{a}^{j}$. Therefore, by the representation of int $\mathbb{R}_{\geq}^{k}$, we have

$$
\left\{a^{1}, \ldots, a^{k}\right\} \subseteq \mathrm{S} .
$$

The examples in the following sections of this paper will show that it even holds $\mathrm{S}=\operatorname{conv}\left\{a^{1}, \ldots, a^{k}\right\}$.

\section{SCALARIZATION}

The purpose of this section is to investigate scalarization methods for vector variational inequality (2.1). Similar to the field of multi-objective optimization, one of the most powerful and most used approaches for vector variational inequalities is to apply a scalarization technique; see, for example, $[35,40,53,55,57,58,60,82,105,111,115,146,140,141,177,182,193]$ and the references therein. These methods are very important from the theoretical as well as computational point of view. Indeed, both necessary and sufficient optimality conditions and even equivalent formulations for problem (2.1) can be derived. To be precise, assume that assumption (A) holds and let $F: X \rightarrow \mathrm{L}(X, Y)$. Further, let

$$
\psi: Y \rightarrow \mathbb{R}
$$

be given. Then, the scalar variational inequality with respect to the scalarization function $\psi$ reads: find an element $x=x(\psi) \in C$ such that

$$
\psi\left(\langle F x, y-x\rangle_{Y}\right) \geq 0, \quad \text { for every } \quad y \in C .
$$

In the context of vector variational inequalities, two types of scalarizing functions have turned out to be of great use:

1. Linear functionals: $\psi: Y \rightarrow \mathbb{R}$ is a linear and continuous function. 
2. Non-linear functions: $\psi: Y \rightarrow \mathbb{R}$ is non-linear and continuous but enjoys the representability condition $\{y \in Y \mid \psi(y)<0\}=-\operatorname{int} K$.

Since scalar variational inequalities are widely developed, scalarization turns out to be of great importance because one can use several methods and techniques from this field in order to study vector variational inequalities.

3.1. Linear scalarization. This section is devoted to the study of linear scalarization techniques for problem (2.1). Let us therefore assume again that assumption (A) holds and let $F: X \rightarrow \mathrm{L}(X, Y)$ be a given mapping. Let $s \in Y^{*}$ be a scalarizing functional, that is,

$$
s: Y \rightarrow \mathbb{R}
$$

is a linear and continuous function. In what follows, it will turn out that taking the zero functional does not make sense; we therefore claim $s \neq 0$. Note that for every $x \in X$, the composition $s \circ F x$ belongs to $X^{*}$, that is, $s \circ F$ defines an operator from $X$ to $X^{*}$. Thus, the scalar variational inequality with respect to the scalarizing functional $s \in Y^{*} \backslash\{0\}$ consists of finding an element $x=x(s) \in C$ such that

$$
\langle s \circ F x, y-x\rangle_{X^{*}, X} \geq 0, \quad \text { for every } \quad y \in C .
$$

Recall that $\langle\cdot, \cdot\rangle_{X^{*}, X}$ denotes the duality pairing between $X$ and $X^{*}$. In what follows, the solution set of problem (3.1) with respect to $s \in Y^{*} \backslash\{0\}$ will be denoted by Sol $\left(\mathrm{VI}_{S}\right)$, that is, we define

$$
\operatorname{Sol}\left(\mathrm{VI}_{s}\right):=\left\{x \in C \mid\langle s \circ F x, y-x\rangle_{X^{*}, X} \geq 0 \text { for every } y \in C\right\} .
$$

In a similar manner, if $X$ and $Y$ are finite-dimensional real Euclidean spaces, say $X=\mathbb{R}^{l}$ and $Y=\mathbb{R}^{k}$, and the cone is given by $K=\mathbb{R}_{\geq}^{k}$, the finite-dimensional scalar variational inequality with respect to the vector $s=\left(s_{1}, \ldots, s_{k}\right)^{\top} \in \mathbb{R}^{k} \backslash\{0\}$ consists of finding an element $x=x(s) \in C$ such that

$$
\left\langle\sum_{j=1}^{k} s_{j} F_{j} x, y-x\right\rangle \geq 0, \quad \text { for every } \quad y \in C .
$$

Recall that in this setting $\left(\mathbb{R}^{k}\right)^{*}=\mathbb{R}^{k}$ and $\langle\cdot, \cdot\rangle$ denotes the Euclidean scalar product in $\mathbb{R}^{l}$. The solution set of problem (3.2) with respect to $s \in \mathbb{R}^{k} \backslash\{0\}$ will be denoted by Sol $\left(\mathrm{VI}_{s}^{\prime}\right)$. For $j=1, \ldots, k$ we denote the $j$ th unit vector in $\mathbb{R}^{k}$ by $\mathrm{e}_{j}$. Evidently, by putting $s=\mathrm{e}_{j}$, the scalar problem (3.2) becomes: find an element $x=x\left(\mathrm{e}_{j}\right)$ such that

$$
\left\langle F_{j} x, y-x\right\rangle \geq 0, \quad \text { for every } \quad y \in C \text {. }
$$

We therefore have

$$
\bigcup_{j=1}^{k} \operatorname{Sol}\left(\mathrm{VI}_{\mathrm{e}_{j}}^{\prime}\right) \subseteq \operatorname{Sol}\left(\mathrm{VVI}^{\prime}\right),
$$

compare the comments in Section 2.4. Motivated by the finite-dimensional case above, some obvious questions are as follows:

1. Is it possible to find $s \in Y^{*} \backslash\{0\}$ such that $\operatorname{Sol}\left(\mathrm{VI}_{s}\right) \subseteq \operatorname{Sol}(\mathrm{VVI})$ ?

2. Conversely, is it possible to find $s \in Y^{*} \backslash\{0\}$ such that $\operatorname{Sol}(\mathrm{VVI}) \subseteq \operatorname{Sol}\left(\mathrm{VI}_{s}\right)$ ?

The next result gives a positive answer to the previous two questions.

Proposition 3.1 ([140, Proposition 2.1]). Besides assumption (A), let $F: X \rightarrow \mathrm{L}(X, Y)$ be a given mapping and assume that the quasi-interior of $K^{*}$ is non-empty. Then it holds

$$
\bigcup_{s \in \mathrm{qi} K^{*}} \operatorname{Sol}\left(\mathrm{VI}_{s}\right) \subseteq \operatorname{Sol}(\mathrm{VVI})=\bigcup_{s \in K^{*} \backslash\{0\}} \operatorname{Sol}\left(\mathrm{VI}_{s}\right) .
$$


Proof. (I). Assume there is $s \in$ qi $K^{*}$ such that $\operatorname{Sol}\left(\mathrm{VI}_{s}\right) \neq \emptyset$ but $\operatorname{Sol}\left(\mathrm{VI}_{s}\right) \nsubseteq \subseteq \operatorname{Sol}(\mathrm{VVI})$. In other words, there is an element $x \in \operatorname{Sol}\left(\mathrm{VI}_{s}\right)$ such that $\langle F x, y-x\rangle_{Y} \in-\operatorname{int} K$ for some $y \in C$. Thus, $\langle F x, y-x\rangle_{Y} \in$ $-K \backslash\{0\}$. However, since $s \in$ qi $K^{*}$, the previous relation shows

$$
\langle s \circ F x, y-x\rangle_{X^{*}, X}<0,
$$

contradicting the fact that $x \in \operatorname{Sol}\left(\mathrm{VI}_{s}\right)$. We therefore have

$$
\bigcup_{s \in \mathrm{qi} K^{*}} \operatorname{Sol}\left(\mathrm{VI}_{s}\right) \subseteq \operatorname{Sol}(\mathrm{VVI})
$$

(II). By using similar arguments, it is easily seen that

$$
\operatorname{Sol}(\mathrm{VVI}) \supseteq \bigcup_{s \in K^{*} \backslash\{0\}} \operatorname{Sol}\left(\mathrm{VI}_{s}\right)
$$

In order to show the reverse inclusion, let $x \in C$ be a solution of problem (2.1). This obviously implies

$$
\langle F x, x\rangle_{Y} \notin\langle F x, C\rangle_{Y}+\operatorname{int} K,
$$

where we define the convex set $\langle F x, C\rangle_{Y}:=\left\{\langle F x, y\rangle_{Y} \mid y \in C\right\}$. Note that the right-hand side of (3.3) is a convex set with non-empty interior. By a separation theorem for convex sets [126], we can find a functional $s \in Y^{*} \backslash\{0\}$ such that

$$
s\left(\langle F x, x\rangle_{Y}\right) \leq s(z)+s(k), \quad \text { for every } \quad z \in\langle F x, C\rangle_{Y}, k \in \operatorname{int} K .
$$

Since $s$ is continuous and the closure of int $K$ is equal to $K$, see Remark 1.6 in [12], it holds

$$
\langle s \circ F x, x\rangle_{X^{*}, X} \leq\langle s \circ F x, y\rangle_{X^{*}, X}+\langle s, k\rangle_{Y^{*}, Y}, \quad \text { for every } \quad y \in C, k \in K .
$$

The previous inequality shows that $s$ belongs to the dual cone of $K$, which implies that $x$ is a solution of problem (3.1) with respect to the functional $s$. The proof is complete.

Remark 3.1. (i) In [140], the authors replace qi $K^{*}$ by int $K^{*}$. However, the sets coincide provided $Y$ enjoys additional properties; see Lemma 2.1.

(ii) Proposition 3.1 shows that vector variational inequality (2.1) can be completely characterized by scalar problems (3.1). It further states necessary and sufficient conditions for problem (2.1).

(iii) Let us consider the finite-dimensional case, where $X=\mathbb{R}^{l}, Y=\mathbb{R}^{k}$, and the cone is given by $K=\mathbb{R}_{\geq}^{k}$. It holds Sol $\left(\mathrm{VI}_{s}\right)=\operatorname{Sol}\left(\mathrm{VI}_{\lambda s}\right)$ for every $\lambda>0$ and $s \in \mathbb{R}_{\geq}^{k} \backslash\{0\}$, which can be checked easily. Therefore, Proposition 3.1 may be stated as

$$
\bigcup_{s \in \operatorname{int} S} \operatorname{Sol}\left(\mathrm{VI}_{s}^{\prime}\right) \subseteq \operatorname{Sol}\left(\mathrm{VVI}^{\prime}\right)=\bigcup_{s \in S} \operatorname{Sol}\left(\mathrm{VI}_{s}^{\prime}\right),
$$

where we define $S:=\left\{s \in \mathbb{R}_{\geq}^{k} \mid\|s\|=1\right\}$ and $\|\cdot\|$ denotes any norm in $\mathbb{R}^{k}$, compare Theorem 2.1 in [141].

(iv) If in addition to the setting of (ii), the set $C \subseteq \mathbb{R}^{l}$ is polyhedral, that is, $C$ is the intersection of finitely many closed half-spaces of $\mathbb{R}^{l}$, then the first inclusion in (3.4) holds as equality; see [156, Theorem 2.1].

Example 3.1. We proceed with Example 2.2. In this example, we are going to study the scalar problems of (2.3) and apply Proposition 3.1 to characterize the solution set of problem (2.3). Let us study the corresponding scalar variational inequalities (3.2) first. For this purpose let $s \in \mathbb{R}_{\geq}^{k} \backslash\{0\}$. Thus, the scalar variational inequality with respect to $s$ consists of finding an element $x \in \mathbb{R}^{l}$ such that

$$
\left\langle\sum_{j=1}^{k} s_{j}\left(x-a^{j}\right), y-x\right\rangle \geq 0, \quad \text { for every } \quad y \in \mathbb{R}^{l} .
$$


It is not hard to check that the element

$$
x=\frac{\sum_{j=1}^{k} s_{j} a^{j}}{\sum_{j=1}^{k} s_{j}}=\sum_{j=1}^{k} \frac{s_{j}}{\sum_{i=1}^{k} s_{i}} a^{j}
$$

is the unique solution of the scalar problem (3.5) with respect to $s \in \mathbb{R}_{\geq}^{k} \backslash\{0\}$. Indeed, this follows from the fact that $x \mapsto \sum_{j=1}^{k} s_{j}\left(x-a^{j}\right)$ is a strictly monotone mapping from $\mathbb{R}^{l}$ to $\mathbb{R}^{l}$. Recall that we denote the solution set of problem (2.3) by S. In what follows, we are going to show that it holds

$$
\mathrm{S}=\operatorname{conv}\left\{a^{1}, \ldots, a^{k}\right\} .
$$

(I). Let us show $\mathrm{S} \subseteq \operatorname{conv}\left\{a^{1}, \ldots, a^{k}\right\}$ first. Denoting the solution set of problem (3.5) with respect to $s \in \mathbb{R}_{\geq}^{k} \backslash\{0\}$ by $\mathrm{S}(s)$, it is easily seen from (3.6) that we have

$$
\mathrm{S}(s) \subseteq \operatorname{conv}\left\{\left\{a^{1}, \ldots, a^{k}\right\} \backslash \bigcup_{j=1}^{k} A_{j}(s)\right\}
$$

where we define for $1 \leq j \leq k$

$$
A_{j}(s):=\left\{a^{j}\right\} \quad \text { if } \quad s_{j}=0 \quad \text { and } \quad A_{j}(s):=\emptyset \quad \text { else. }
$$

Applying Proposition 3.1, we obtain

$$
\mathrm{S}=\bigcup_{s \in \mathbb{R}_{\geq}^{k} \backslash\{0\}} \mathrm{S}(s) \subseteq \bigcup_{s \in \mathbb{R}_{\geq}^{k} \backslash\{0\}} \operatorname{conv}\left\{\left\{a^{1}, \ldots, a^{k}\right\} \backslash \bigcup_{j=1}^{k} A_{j}(s)\right\} \subseteq \operatorname{conv}\left\{a^{1}, \ldots, a^{k}\right\} .
$$

(II). Let us show the converse inclusion $\mathrm{S} \supseteq \operatorname{conv}\left\{a^{1}, \ldots, a^{k}\right\}$. Therefore let $x$ be an arbitrarily chosen element in the convex hull of $a^{1}, \ldots, a^{k}$. Thus, we can find real numbers $\lambda_{1}, \ldots, \lambda_{k}$ with $\lambda_{j} \geq 0$ for $j=1, \ldots, k$ and $\sum_{j=1}^{k} \lambda_{j}=1$ such that

$$
x=\sum_{j=1}^{k} \lambda_{j} a^{j} .
$$

Without any loss of generality, let $\lambda_{j} \neq 0$ for $j=1, \ldots, k$. Assuming by contradiction that $x$ does not solve problem (2.3), there exists an element $y \in \mathbb{R}^{l}$ such that

$$
\left\langle x-a^{j}, y-x\right\rangle<0, \quad \text { for } j=1, \ldots, k .
$$

However, by multiplying every inequality with $\lambda_{j}$ and summing up the resulting inequalities, we obtain

$$
0<\sum_{j=1}^{k} \lambda_{j}\left\langle x-a^{j}, y-x\right\rangle=\sum_{j=1}^{k} \lambda_{j}\langle x, y-x\rangle-\sum_{j=1}^{k} \lambda_{j}\left\langle a^{j}, y-x\right\rangle=\langle x, y-x\rangle-\langle x, y-x\rangle=0,
$$

which is impossible. This shows (3.7).

We have already seen that every scalar variational inequality (3.5) has a unique solution. In other words, for every $s \in \mathbb{R}_{\geq}^{k} \backslash\{0\}$, the set $\mathrm{S}(s)$ is a singleton. In what follows, we will characterize certain unions of solution sets to get a better understanding of the scalar problems. Relation (3.8) already indicates that the solution of problem (3.5) depends on the number and position of entries of $s \in \mathbb{R}_{\geq}^{k} \backslash\{0\}$, which are non-zero. We therefore define

$$
I(s):=\left\{j \in\{1, \ldots, k\} \mid s_{j} \neq 0\right\} \quad \text { and } \quad \kappa(s):=\# I(s) .
$$

Namely, the set and number of non-zero entries of $s$, respectively. Let us further define

$$
Z(s):=\left\{\tilde{s} \in \mathbb{R}_{\geq}^{k} \mid I(s)=I(\tilde{s}) \text { and } \kappa(s)=\kappa(\tilde{s})\right\} .
$$


In the following, we denote the non-zero entries of $s$ by $j_{1}(s), \ldots, j_{\kappa(s)}(s)$. Note that it holds $\kappa(s) \in$ $\{1, \ldots, k\}$ for every $s \in \mathbb{R}_{\geq}^{k} \backslash\{0\}$ and $Z(s)=\operatorname{int} \mathbb{R}_{\geq}^{k}$ provided $\kappa(s)=k$. Using relation (3.8), it is easily seen that for every $s \in \mathbb{R}_{\geq}^{k} \backslash\{0\}$ it holds

$$
\bigcup_{\tilde{s} \in Z(s)} \mathrm{S}(\tilde{s})=\operatorname{conv}\left\{a^{j_{1}(s)}, \ldots, a^{j_{\kappa(s)}(s)}\right\}
$$

Therefore, using the above representation and Proposition 3.1, we obtain

$$
\begin{aligned}
\mathrm{S}=\bigcup_{s \in \mathbb{R}_{\geq}^{k} \backslash\{0\}} \mathrm{S}(s) & =\bigcup_{s \in \mathbb{R}_{\geq}^{k} \backslash\{0\}} \bigcup_{s \in Z(s)} \mathrm{S}(\tilde{s}) \\
& =\bigcup_{s \in \mathbb{R}_{\geq}^{k} \backslash\{0\}} \operatorname{conv}\left\{a^{j_{1}(s)}, \ldots, a^{j_{\kappa(s)}(s)}\right\}=\operatorname{conv}\left\{a^{1}, \ldots, a^{k}\right\},
\end{aligned}
$$

which confirms (3.7) once again. Note, that if $l=2$ and $k=5$, for instance, then (3.9) recovers the following representation of unions of scalar variational inequalities:

1. For $s \in \mathbb{R}_{\geq}^{5} \backslash\{0\}$ with $\kappa(s)=1$, we have

$$
\bigcup_{\tilde{s} \in Z(s)} \mathrm{S}(\tilde{s})=\bigcup_{\tilde{s}_{j_{1}(s)} \in \mathbb{R}_{>}} \mathrm{S}(\tilde{s})=\left\{a^{j_{1}(s)}\right\} .
$$

2. For $s \in \mathbb{R}_{\geq}^{5} \backslash\{0\}$ with $\kappa(s)=2$, we have

$$
\bigcup_{\tilde{s} \in Z(s)} \mathrm{S}(\tilde{s})=\bigcup_{\tilde{s}_{j_{1}(s)}, \tilde{s}_{j_{2}(s)} \in \mathbb{R}_{>}} \mathrm{S}(\tilde{s})=\left\{a^{j_{1}(s)}, a^{j_{2}(s)}\right\} .
$$

3. For $s \in \mathbb{R}_{\geq}^{5} \backslash\{0\}$ with $\kappa(s)=3$, we have

$$
\bigcup_{\tilde{s} \in Z(s)} \mathrm{S}(\tilde{s})=\bigcup_{\tilde{s}_{j_{1}(s)}, \tilde{s}_{j_{2}(s)}, \tilde{s}_{j_{3}(s)} \in \mathbb{R}_{>}} \mathrm{S}(\tilde{s})=\left\{a^{j_{1}(s)}, a^{j_{2}(s)}, a^{j_{3}(s)}\right\} .
$$

4. For $s \in \mathbb{R}_{\geq}^{5} \backslash\{0\}$ with $\kappa(s)=4$, we have

$$
\bigcup_{\tilde{s} \in Z(s)} \mathrm{S}(\tilde{s})=\bigcup_{\tilde{s}_{j_{1}(s)}, \tilde{s}_{j_{2}(s)}, \tilde{s}_{j_{3}(s)}, \tilde{s}_{j_{4}(s)} \in \mathbb{R}_{>}} \mathrm{S}(\tilde{s})=\left\{a^{j_{1}(s)}, a^{j_{2}(s)}, a^{j_{3}(s)}, a^{j_{4}(s)}\right\} .
$$

5. For $s \in \mathbb{R}_{\geq}^{5} \backslash\{0\}$ with $\kappa(s)=5$, we have

$$
\bigcup_{\tilde{s} \in Z(s)} \mathrm{S}(\tilde{s})=\bigcup_{\tilde{s}_{1}, \tilde{s_{2}}, \tilde{s_{3}}, \tilde{s}_{4}, \tilde{s}_{5} \in \mathbb{R}_{>}} \mathrm{S}(\tilde{s})=\left\{a^{1}, a^{2}, a^{3}, a^{4}, a^{5}\right\}
$$

Remark 3.2. The previous example shows that we need to calculate the solution set of all scalar problems in order to cover the complete solution set of problem (2.3).

3.2. Non-linear scalarization. This section is intended as an attempt at motivating the power of nonlinear scalarizing functions. Indeed, Proposition 3.1 and Example 3.1 lead us to assume that, by using only one linear and continuous scalarizing functional, one is not able to cover the whole solution set of problem (2.1). In general, we have

$$
\operatorname{Sol}\left(\mathrm{VI}_{s}\right) \subsetneq \operatorname{Sol}(\mathrm{VI})
$$

where $s \in K^{*} \backslash\{0\}$; compare Example 3.1. It is even possible that the solution set $\mathrm{Sol}\left(\mathrm{VI}_{s}\right)$ is empty such that the previous inclusion becomes trivial. However, it turns out that by using a certain non-linear scalarizing function, the resulting scalar problem is equivalent to problem (2.1) in the sense that their solution sets coincide, provided the representability condition is satisfied. Therefore, by solving only one scalar problem, using a non-linear scalarizing function, we cover the whole solution set of problem 
(2.1). To be precise, assume again that assumption (A) holds and let $e \in \operatorname{int} K$ be arbitrarily chosen. Denote by

$$
\varphi^{K, e}: Y \rightarrow \mathbb{R}
$$

the non-linear Tammer-Weidner function, introduced in Definition 2.14, and recall that $\varphi^{K, e}$ enjoys the representability condition

$$
\left\{y \in Y \mid \varphi^{K, e}(y)<0\right\}=-\operatorname{int} K
$$

compare Theorem 2.4. Therefore, the following scalar variational inequality with respect to the nonlinear Tammer-Weidner function $\varphi^{K, e}$ is equivalent to problem (2.1): find an element $x=x\left(\varphi^{K, e}\right) \in C$ such that

$$
\varphi^{K, e}\left(\langle F x, y-x\rangle_{Y}\right) \geq 0, \quad \text { for every } \quad y \in C .
$$

The above formulation has been frequently used in the literature to derive existence results for (generalized) vector variational inequalities; compare Section 5 and the references [40, 53]. Note that in [55], the authors use a modified non-linear scalarization function for quasi-vector equilibrium problems.

Remark 3.3. Over the years, several scalarizing functions have been introduced and employed in functional analysis, risk theory, and many others. In a recent paper, Bouza et al. give a unified point of view to the known scalarizing functions in optimization; see [26]. In their paper, the authors consider three different classes: the class of separating functionals with uniform level sets (Tammer-Weidner functions), the class of Hiriart-Urruty functions and the class of Drummond-Svaiter functions.

Example 3.2. We proceed with Example 3.1. Let $e \in \operatorname{int} \mathbb{R}_{\geq}^{k}$ be arbitrarily chosen. Note that for every $x, y \in \mathbb{R}^{l}$, it holds

$$
\begin{aligned}
\varphi^{\mathbb{R}_{\geq}^{k}, e}\left(\langle F x, y-x\rangle_{\mathbb{R}_{\geq}^{k}}\right) & =\min \left\{t \in \mathbb{R} \mid\left(\begin{array}{c}
\left\langle x-a^{1}, y-x\right\rangle \\
\vdots \\
\left\langle x-a^{k}, y-x\right\rangle
\end{array}\right) \in t\left(\begin{array}{c}
e_{1} \\
\vdots \\
e_{k}
\end{array}\right)-\mathbb{R}_{\geq}^{k}\right\} \\
& =\min \left\{t \in \mathbb{R} \mid \frac{\left\langle x-a^{j}, y-x\right\rangle}{e_{j}} \leq t \text { for } j=1, \ldots, k\right\} \\
& =\max _{j=1, \ldots, k}\left\langle x-a^{j}, y-x\right\rangle .
\end{aligned}
$$

Thus, the non-linear scalar variational inequality (3.10) becomes: find an element $x \in \mathbb{R}^{l}$ such that

$$
\max _{j=1, \ldots, k}\left\langle x-a^{j}, y-x\right\rangle \geq 0, \quad \text { for every } \quad y \in \mathbb{R}^{l} .
$$

Note that problem (3.11) does not depend upon the element $e$. In what follows, we are going to denote the solution set of problem (3.11) by S. Evidently, by the representability condition of the Tammer-Weidner function and Example 3.1, the solution of (3.11) is equivalent to the convex hull of $a^{1}, \ldots, a^{k}$. However, let us again verify, using the above non-linear scalar variational inequality, that (3.7) holds. Clearly, it is enough to prove

$$
\tilde{\mathrm{S}}=\operatorname{conv}\left\{a^{1}, \ldots, a^{k}\right\} .
$$

(I). Let us show $\tilde{\mathrm{S}} \subseteq \operatorname{conv}\left\{a^{1}, \ldots, a^{k}\right\}$ first. Indeed, let $x \in \tilde{\mathrm{S}}$ be arbitrarily chosen. Then we can find a non-empty set $P \subseteq\{1, \ldots, k\}$, depending on the element $x$, such that

$$
\left\langle x-a^{j}, y-x\right\rangle \geq 0, \quad \text { for every } \quad j \in P, y \in \mathbb{R}^{l} .
$$


Without any loss of generality, we may assume that $P=\{1, \ldots, p\}$ for some $p \in\{1, \ldots, k\}$. Now let $\lambda_{1}, \ldots, \lambda_{p}$ be arbitrarily chosen numbers with $\lambda_{j} \geq 0$ for $j=1, \ldots, p$ and $\sum_{j=1}^{p} \lambda_{j}=1$. Multiplying every inequality by $\lambda_{j}$ and summing them up, we obtain

$$
0 \leq \sum_{j=1}^{p} \lambda_{j}\left\langle x-a^{j}, y-x\right\rangle=\left\langle x-\sum_{j=1}^{p} \lambda_{j} a^{j}, y-x\right\rangle, \quad \text { for every } \quad y \in \mathbb{R}^{l} .
$$

However, similar to the previous example, the unique solution of the above variational inequality is given by

$$
x=\sum_{j=1}^{p} \lambda_{j} a^{j} \in \operatorname{conv}\left\{a^{1}, \ldots, a^{p}\right\} \subseteq \operatorname{conv}\left\{a^{1}, \ldots, a^{k}\right\} .
$$

(II). Let us show $\tilde{\mathrm{S}} \supseteq \operatorname{conv}\left\{a^{1}, \ldots, a^{k}\right\}$. Indeed, let $x \in \operatorname{conv}\left\{a^{1}, \ldots, a^{k}\right\}$ be arbitrarily chosen. Then there are real numbers $\lambda_{1}, \ldots, \lambda_{k}$ with $\lambda_{j} \geq 0$ for $j=1, \ldots, k$ and $\sum_{j=1}^{k} \lambda_{j}=1$ such that $x=\sum_{j=1}^{k} \lambda_{j} a^{j}$. Assume by contradiction that $x \notin \tilde{\mathrm{S}}$. Then there is $y \in \mathbb{R}^{l}$ such that

$$
\left\langle x-a^{j}, y-x\right\rangle<0, \quad \text { for } \quad j=1, \ldots, k \text {. }
$$

This is impossible, compare Example 3.1, which shows (3.12). The previous calculations again verify, by solving the scalar problem (3.11) only, that the solution set of problem (2.3) is given by $\operatorname{conv}\left\{a^{1}, \ldots, a^{k}\right\}$.

3.3. Pareto reducibility. The present section is devoted to the notion of Pareto reducibility of the finite-dimensional vector variational inequality (2.2), which has recently been introduced by Popovici an Rocca; see [177]. It should be noted that the concept of Pareto reducibility has also been introduced in [178] for multi-objective optimization problems. Let us recall that the finite-dimensional vector variational inequality (2.2) consists of finding an element $x \in C$ such that

$$
\langle F x, y-x\rangle_{\mathbb{R}^{k}} \notin-\operatorname{int} \mathbb{R}_{\geq}^{k}, \quad \text { for every } \quad y \in C,
$$

where $C$ is a non-empty, closed and convex subset of $\mathbb{R}^{l}$ and $F: \mathbb{R}^{l} \rightarrow \operatorname{Mat}_{k \times l}(\mathbb{R})$ is a given mapping. Roughly spoken, the key idea in [177] is to replace (2.2) by a finite family of subproblems. For this purpose, we introduce the following strong finite-dimensional vector variational inequality: find an element $x \in C$ such that

$$
\langle F x, y-x\rangle_{\mathbb{R}^{k}} \notin-\mathbb{R}_{\geq}^{k} \backslash\{0\}, \quad \text { for every } \quad y \in C .
$$

In order to introduce the subproblems of (3.13), define the index set

$$
J_{k}:=\{1, \ldots, k\} .
$$

For any subset $J=\left\{j_{1}, \ldots j_{m}\right\} \subseteq J_{k}$, where $1 \leq m \leq k$, for any $x \in \mathbb{R}^{l}$ and $y \in \mathbb{R}^{k}$, we define

$$
\langle F x, y\rangle_{J}:=\left(\left\langle F_{j_{1}} x, y\right\rangle, \ldots,\left\langle F_{j_{m}} x, y\right\rangle\right) \in \mathbb{R}^{m},
$$

where $\langle\cdot, \cdot\rangle$ denotes the Euclidean scalar product in $\mathbb{R}^{l}$. Note that $\langle F x, y\rangle_{J_{k}}=\langle F x, y\rangle_{\mathbb{R}^{k}}$. Thus, using the above notion, the subproblem of (3.13) with respect to $J=\left\{j_{1}, \ldots j_{m}\right\}$ consists of finding an element $x \in C$ such that

$$
\langle F x, y-x\rangle_{J} \notin-\mathbb{R}_{\geq}^{m} \backslash\{0\}, \quad \text { for every } \quad y \in C .
$$

In what follows, we denote the solution set of problem (3.14) by $\operatorname{Sol}\left(\mathrm{SVVI}_{J}^{\prime}\right)$, that is,

$$
\operatorname{Sol}\left(\mathrm{SVVI}_{J}^{\prime}\right):=\left\{x \in C \mid\langle F x, y-x\rangle_{J} \notin-\mathbb{R}_{\geq}^{m} \backslash\{0\} \text { for every } y \in \mathbb{R}^{l}\right\} .
$$

Note, that if $J=\{j\}$ is a singleton for some $j \in\{1, \ldots, k\}$, then we have

$$
\operatorname{Sol}\left(\operatorname{SVVI}_{J}^{\prime}\right)=\operatorname{Sol}\left(\mathrm{VI}_{\mathrm{e}_{j}}^{\prime}\right) \text {. }
$$

Here, $\mathrm{e}_{j}$ denotes the $j$ th unit vector in $\mathbb{R}^{k}$. 
The next result shows that the solution set of problem (2.2) can be decomposed using subproblems.

Theorem 3.1 ([177, Theorem 1]). Besides assumption (A), let $F: \mathbb{R}^{l} \rightarrow \operatorname{Mat}_{k \times l}(\mathbb{R})$ be a given mapping. Then, it holds

$$
\operatorname{Sol}\left(\mathrm{VVI}^{\prime}\right)=\bigcup_{\emptyset \neq J \subseteq J_{k}} \operatorname{Sol}\left(\mathrm{SVVI}_{J}^{\prime}\right)
$$

Proof. Let us show the inclusion $\subseteq$ in (3.15) first. We therefore have to find a non-empty subset $\tilde{J}$ of $J_{k}$ such that $\operatorname{Sol}\left(\mathrm{VVI}^{\prime}\right) \subseteq \operatorname{Sol}\left(\mathrm{SVVI}_{\tilde{J}}^{\prime}\right)$. Let $x \in \operatorname{Sol}\left(\mathrm{VVI}^{\prime}\right)$ be arbitrarily chosen. By Proposition 3.1, there exists a vector $s \in \mathbb{R}_{\geq}^{k} \backslash\{0\}$ such that

$$
\left\langle\sum_{j=1}^{k} s_{j} F_{j} x, y-x\right\rangle \geq 0, \quad \text { for every } \quad y \in C .
$$

In what follows, we will consider the non-empty set $\tilde{J}:=\left\{j \in J_{k} \mid s_{j}>0\right\}$. Without any loss of generality assume that $\tilde{J}=\left\{j_{1}, \ldots, j_{p}\right\}$ for some $1 \leq p \leq k$. By defining the vector $\tilde{s} \in \operatorname{int} \mathbb{R}_{\geq}^{p}$ by $\tilde{s}_{j}=s_{j}$ for every $j \in\left\{j_{1}, \ldots, j_{p}\right\}$, the above scalar problem may be written as

$$
\left\langle\sum_{r=1}^{p} \tilde{s}_{j_{r}} F_{j_{r}} x, y-x\right\rangle \geq 0, \quad \text { for every } \quad y \in C .
$$

Let us define $\operatorname{Sol}\left(\mathrm{VI}_{\tilde{J}, s}\right):=\left\{x \in C \mid\left\langle\sum_{r=1}^{p} s_{j_{r}} F_{j_{r}} x, y-x\right\rangle \geq 0\right.$, for every $\left.y \in C\right\}$ for any vector $s \in \mathbb{R}_{\geq}^{k} \backslash\{0\}$. Then, Proposition 3.1 implies

$$
\operatorname{Sol}\left(\operatorname{VI}_{\tilde{J}, \tilde{s}}\right) \subseteq \bigcup_{s \in \operatorname{int} \mathbb{R}_{\geq}^{k}} \operatorname{Sol}\left(\mathrm{VI}_{\tilde{J}, s}\right) \subseteq \operatorname{Sol}\left(\operatorname{SVVI}_{\tilde{J}}^{\prime}\right)
$$

The above line shows that the inclusion $\subseteq$ in (3.15) holds. However, the reverse inclusion $\supseteq$ in (3.15) can be shown by using similar arguments. The main tool is again Proposition 3.1. The proof is complete.

Example 3.3. We proceed with Example 3.2. Recall that the finite-dimensional vector variational inequality (2.3) consists of finding an element $x \in \mathbb{R}^{l}$ such that

$$
\left(\begin{array}{c}
\left\langle x-a^{1}, y-x\right\rangle \\
\vdots \\
\left\langle x-a^{k}, y-x\right\rangle
\end{array}\right) \notin-\operatorname{int} \mathbb{R}_{\geq}^{k}, \quad \text { for every } \quad y \in \mathbb{R}^{l} .
$$

In what follows, we are again going to show that the solution set $S$ of problem (2.3) is equivalent to the convex hull of $a^{1}, \ldots, a^{k}$, using Theorem 3.1. Therefore, let $J=\left\{j_{1}, \ldots, j_{m}\right\} \subseteq J_{k}$ be arbitrarily chosen and consider the following subproblem: find an element $x \in \mathbb{R}^{l}$ such that

$$
\left(\begin{array}{c}
\left\langle x-a^{j_{1}}, y-x\right\rangle \\
\vdots \\
\left\langle x-a^{j_{m}}, y-x\right\rangle
\end{array}\right) \notin-\mathbb{R}_{\geq}^{m} \backslash\{0\}, \quad \text { for every } \quad y \in \mathbb{R}^{l} .
$$

Let us denote the solution set of the above subproblem by $\mathrm{S}(J)$. In the following, we are going to show that it holds

$$
\mathrm{S}(J)=\operatorname{conv}\left\{a^{j_{1}}, \ldots, a^{j_{m}}\right\} .
$$

(I). We first show $\mathrm{S}(J) \subseteq \operatorname{conv}\left\{a^{j_{1}}, \ldots, a^{j_{m}}\right\}$. Indeed, let $x \in \mathbb{R}^{l}$ be a solution of the subproblem with respect to $J=\left\{j_{1}, \ldots, j_{m}\right\}$. Thus, we can find disjoint subsets $P, N \subseteq J$ with $P \cup N=J$ such that

$$
\begin{array}{lll}
\left\langle x-a^{j}, y-x\right\rangle \geq 0, & \text { for every } & y \in \mathbb{R}^{l}, j \in P \\
\left\langle x-a^{j}, y-x\right\rangle<0, & \text { for every } & y \in \mathbb{R}^{l}, j \in N .
\end{array}
$$


Let us put $P=\left\{i_{1}, \ldots, i_{p}\right\}$ for some $p \in\{1, \ldots, m\}$. Now let $\lambda_{i_{1}}, \ldots, \lambda_{i_{p}}$ be positive reals with $\sum_{r=1}^{p} \lambda_{i_{r}}=1$. Multiplying every positive inequality by $\lambda_{i_{r}}$ and summing them up yields

$$
0 \leq \sum_{r=1}^{p} \lambda_{i_{r}}\left\langle x-a^{i_{r}}, y-x\right\rangle=\left\langle x-\sum_{r=1}^{p} \lambda_{i_{r}} a^{i_{r}}, y-x\right\rangle, \quad \text { for every } \quad y \in \mathbb{R}^{l} .
$$

Similar to the previous examples, the unique solution of the above variational inequality is given by

$$
x=\sum_{r=1}^{p} \lambda_{i_{r}} a^{i_{r}} \in \operatorname{conv}\left\{a^{i_{1}}, \ldots, a^{i_{p}}\right\} \subseteq \operatorname{conv}\left\{a^{j_{1}}, \ldots, a^{j_{m}}\right\},
$$

which shows $\mathrm{S}(J) \subseteq \operatorname{conv}\left\{a^{j_{1}}, \ldots, a^{j_{m}}\right\}$.

(II). Let us show $\mathrm{S}(J) \supseteq \operatorname{conv}\left\{a^{j_{1}}, \ldots, a^{j_{m}}\right\}$. Indeed, let $x \in \operatorname{conv}\left\{a^{j_{1}}, \ldots, a^{j_{m}}\right\}$ be arbitrarily chosen. If $x \notin \mathrm{S}(J)$, then we can find some element $y \in \mathbb{R}^{l}$ such that

$$
\left(\begin{array}{c}
\left\langle x-a^{j_{1}}, y-x\right\rangle \\
\vdots \\
\left\langle x-a^{j_{m}}, y-x\right\rangle
\end{array}\right) \in-\mathbb{R}_{\geq}^{m} \backslash\{0\}
$$

However, by adapting the arguments in part (II) of Example 3.1, this is impossible. This shows (3.16). Thus, applying Theorem 3.1, we obtain again

$$
\mathrm{S}=\bigcup_{\emptyset \neq J \subseteq J_{k}} \mathrm{~S}(J)=\operatorname{conv}\left\{a^{1}, \ldots, a^{k}\right\} .
$$

Let us again consider the special case where we let $l=2$ and $k=5$. Thus, there are five types of subproblems:

1. For $J=\{j\}$, where $1 \leq j \leq 5$, the subproblem becomes: find an element $x \in \mathbb{R}^{2}$ such that

$$
\langle F x, y-x\rangle_{J}=\left\langle F_{j} x, y-x\right\rangle \geq 0, \quad \text { for every } y \in \mathbb{R}^{2} .
$$

The solution set is given by $\mathrm{S}(J)=\left\{a^{j}\right\}$.

2. For $J=\left\{j_{1}, j_{2}\right\}$, where $1 \leq j_{1}<j_{2} \leq 5$, the subproblem becomes: find an element $x \in \mathbb{R}^{2}$ such that

$$
\langle F x, y-x\rangle_{J}=\left(\begin{array}{c}
\left\langle F_{j_{1}} x, y-x\right\rangle \\
\left\langle F_{j_{2}} x, y-x\right\rangle
\end{array}\right) \notin-\mathbb{R}_{\geq}^{2} \backslash\{0\}, \quad \text { for every } \quad y \in \mathbb{R}^{2} .
$$

The solution set is given by $\mathrm{S}(J)=\operatorname{conv}\left\{a^{j_{1}}, a^{j_{2}}\right\}$.

3. For $J=\left\{j_{1}, j_{2}, j_{3}\right\}$, where $1 \leq j_{1}<j_{2}<j_{3} \leq 5$, the subproblem becomes: find an element $x \in \mathbb{R}^{2}$ such that

$$
\langle F x, y-x\rangle_{J}=\left(\begin{array}{c}
\left\langle F_{j_{1}} x, y-x\right\rangle \\
\left\langle F_{j_{2}} x, y-x\right\rangle \\
\left\langle F_{j_{3}} x, y-x\right\rangle
\end{array}\right) \notin-\mathbb{R}_{\geq}^{3} \backslash\{0\}, \quad \text { for every } y \in \mathbb{R}^{2} .
$$

The solution set is given by $\mathrm{S}(J)=\operatorname{conv}\left\{a^{j_{1}}, a^{j_{2}}, a^{j_{3}}\right\}$.

4. For $J=\left\{j_{1}, j_{2}, j_{3}, j_{4}\right\}$, where $1 \leq j_{1}<j_{2}<j_{3}<j_{4} \leq 5$, the subproblem becomes: find an element $x \in \mathbb{R}^{2}$ such that

$$
\langle F x, y-x\rangle_{J}=\left(\begin{array}{c}
\left\langle F_{j_{1}} x, y-x\right\rangle \\
\left\langle F_{j_{2}} x, y-x\right\rangle \\
\left\langle F_{j_{3}} x, y-x\right\rangle \\
\left\langle F_{j_{4}} x, y-x\right\rangle
\end{array}\right) \notin-\mathbb{R}_{\geq}^{4} \backslash\{0\}, \quad \text { for every } \quad y \in \mathbb{R}^{2} .
$$

The solution set is given by $\mathrm{S}(J)=\operatorname{conv}\left\{a^{j_{1}}, a^{j_{2}}, a^{j_{3}}, a^{j_{4}}\right\}$. 
5. For $J=J_{5}$, the subproblem becomes: find an element $x \in \mathbb{R}^{2}$ such that

$$
\langle F x, y-x\rangle_{J}=\left(\begin{array}{c}
\left\langle F_{1} x, y-x\right\rangle \\
\left\langle F_{2} x, y-x\right\rangle \\
\left\langle F_{3} x, y-x\right\rangle \\
\left\langle F_{4} x, y-x\right\rangle \\
\left\langle F_{5} x, y-x\right\rangle
\end{array}\right) \notin-\mathbb{R}_{\geq}^{5} \backslash\{0\}, \quad \text { for every } y \in \mathbb{R}^{2} .
$$

The solution set is given by $\mathrm{S}(J)=\operatorname{conv}\left\{a^{1}, a^{2}, a^{3}, a^{4}, a^{5}\right\}$.

In this case, the solution set of the finite-dimensional vector variational inequality (2.3) is given by

$$
\begin{aligned}
& S=\bigcup_{\emptyset \neq J \subseteq J_{5}} S(J)=\bigcup_{j=1}^{5} \bigcup_{\substack{J \subseteq J_{5} \\
\# J=j}} S(J) \\
& =\bigcup_{1 \leq j \leq 5}\left\{a^{j}\right\} \cup \bigcup_{1 \leq j_{1}<j_{2} \leq 5} \operatorname{conv}\left\{a^{j_{1}}, a^{j_{2}}\right\} \cup \underset{1 \leq j_{1}<j_{2}<j_{3} \leq 5}{\bigcup_{1}} \operatorname{conv}\left\{a^{j_{1}}, a^{j_{2}}, a^{j_{3}}\right\} \\
& \cup \bigcup_{1 \leq j_{1}<j_{2}<j_{3}<j_{4} \leq 5} \operatorname{conv}\left\{a^{j_{1}}, a^{j_{2}}, a^{j_{3}}, a^{j_{4}}\right\} \cup \operatorname{conv}\left\{a^{1}, \ldots, a^{5}\right\} \\
& =\operatorname{conv}\left\{a^{1}, \ldots, a^{5}\right\} \text {. }
\end{aligned}
$$

The next figure shows the solution set of all subproblems for the special case $l=2$ and $k=5$. The figures (A) and (B) show the solution set of all subproblems (3.17) and (3.18), respectively. The figures (C) (L) show the solution sets of all subproblems (3.19). The figures (M) and (N) show the solution set of all subproblems (3.20) while figure (O) shows the solution set of subproblem (3.21).

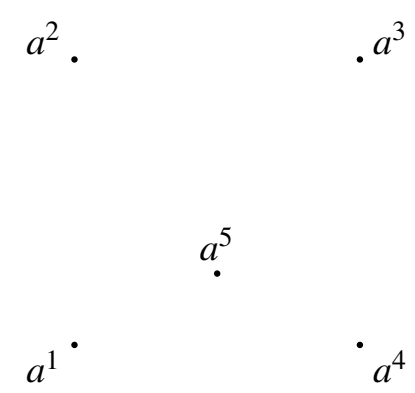

(A)

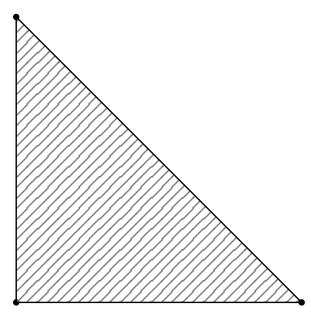

(D)

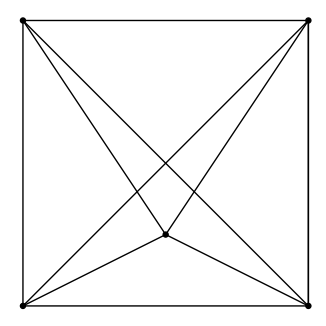

(B)

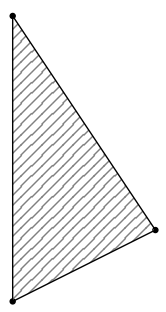

(E)

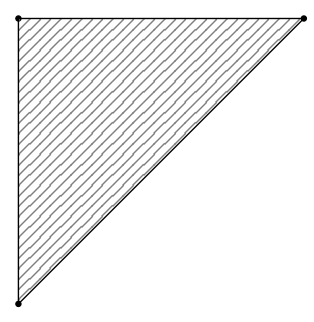

(C)

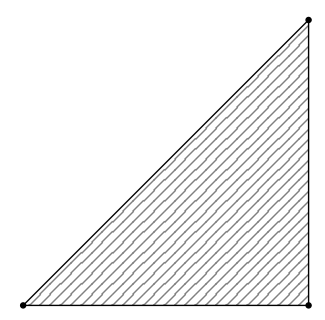

(F) 


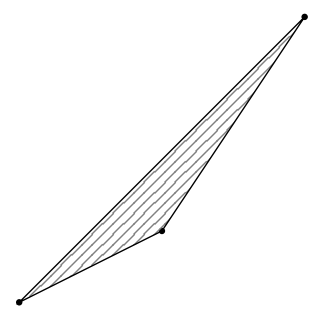

(G)

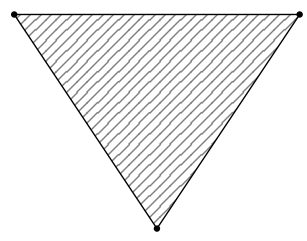

(

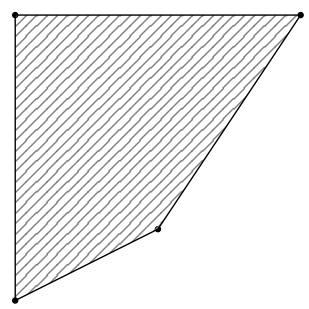

(M)

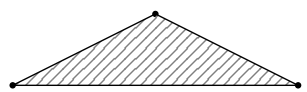

(H)

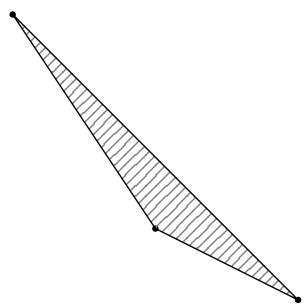

$(\mathrm{K})$

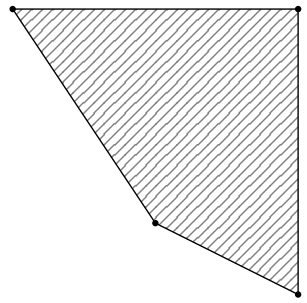

$(\mathrm{N})$

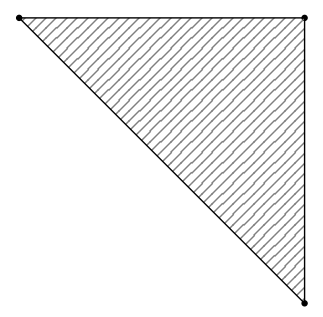

(I)

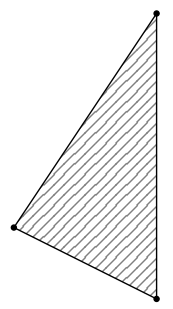

(L)

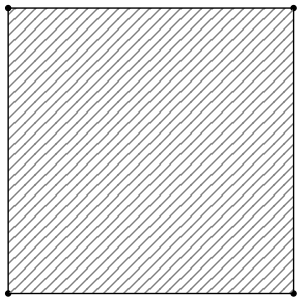

(O)

FIGURE 6. Several solution sets of subproblems.

\section{TOPOLOGICAL AND ALGEBRAIC PROPERTIES OF THE SOLUTION SET}

The purpose of this section is to investigate several topological and algebraical properties of the solution set of vector variational inequalities (2.1) and (2.2), respectively. We will discuss under which conditions of the data, the corresponding solution set is closed, bounded, compact, connected and semialgebraic since properties of this kind will be used frequently in the following sections. However, conditions, which ensure that the solution set is non-empty, will be studied in Section 5 .

4.1. Boundedness, closedness and compactness. In what follows, we are going to study the closedness, boundedness and compactness of the solution set of the problems (2.1) and (2.2), respectively. Therefore, the following definitions will be needed, which can be found, for example, in [12].

Definition 4.1. Besides assumption (A), let $F: X \rightarrow \mathrm{L}(X, Y)$ be a given mapping. $F$ is called 
(i) $K$-monotone if for every $x, y \in X$ it holds

$$
\langle F x-F y, x-y\rangle_{Y} \in K
$$

(ii) $K$-pseudomonotone if for every $x, y \in X$ it holds that $\langle F x, y-x\rangle_{Y} \notin-\operatorname{int} K$ implies $\langle F y, y-x\rangle_{Y} \notin$ $-\operatorname{int} K$;

(iii) $v$-hemicontinuous if for every $x, y, z \in X$ the mapping $\mathbb{R} \rightarrow Y$, given by $t \mapsto\langle F(x+t y), z\rangle_{Y}$, is continuous at $0^{+}$

(iv) continuous at $x \in X$ if for every sequence $\left\{x_{n}\right\} \subseteq X$ with $x_{n} \rightarrow x$ it holds $F x_{n} \rightarrow F x$;

(v) continuous if $F$ is continuous at every point in $X$.

Remark 4.1. (i) Using Proposition 2.5 (iv), it is easily seen that any $K$-monotone mapping is $K$ pseudomonotone.

(ii) Any continuous mapping $F: X \rightarrow \mathrm{L}(X, Y)$ is $v$-hemicontinuous. However, the converse does not hold in general.

(iii) If we let $Y=\mathbb{R}$ and $K=\mathbb{R}_{\geq}$, then the above notions recover the notions of hemicontinuous and monotone operators from $X$ to $\mathrm{L}(X, \mathbb{R})=X^{*}$; see [199]. An operator $A: X \rightarrow X^{*}$ is called monotone if for every $x, y \in X$ it holds that $\langle A x-A y, x-y\rangle_{X^{*}, X} \geq 0$. A is called strictly monotone if for every $x, y \in X$, $x \neq y$, it holds that $\langle A x-A y, x-y\rangle_{X^{*}, X}>0$. $A$ is said to be hemicontinuous if the mapping $\mathbb{R} \rightarrow \mathbb{R}$, given by $t \mapsto\langle A(x+t y), z\rangle_{X^{*}, X}$, is continuous at $0^{+}$.

Example 4.1. Let us show that the mapping $F: \mathbb{R}^{l} \rightarrow \operatorname{Mat}_{k \times l}(\mathbb{R})$, given by (2.4), is $\mathbb{R}_{>}^{k}$-monotone and $v$-hemicontinuous. Indeed, let $x, y, z \in \mathbb{R}^{l}$ be arbitrarily chosen and define $\varphi(t):=\langle F(x+t y), z\rangle_{\mathbb{R}^{k}}$ for $t \in \mathbb{R}$. Then we have

$$
\langle F x-F y, x-y\rangle_{\mathbb{R}^{k}}=\left(\begin{array}{c}
\left\langle x-a^{1}-y+a^{1}, x-y\right\rangle \\
\vdots \\
\left\langle x-a^{k}-y+a^{k}, x-y\right\rangle
\end{array}\right)=\left(\begin{array}{c}
\|x-y\|_{2}^{2} \\
\vdots \\
\|x-y\|_{2}^{2}
\end{array}\right) \in \mathbb{R}_{\geq}^{k},
$$

which shows that $F$ is $\mathbb{R}_{\geq}^{k}$-monotone. Note further that $\varphi(0)=\langle F x, z\rangle_{\mathbb{R}^{k}}$. Hence, we have for every $t \in \mathbb{R}$

$$
\|\varphi(t)-\varphi(0)\|_{2}=\left\|\left(\begin{array}{c}
\langle t y, z\rangle \\
\vdots \\
\langle t y, z\rangle
\end{array}\right)\right\|_{2}=|t| \sqrt{k}|\langle y, z\rangle|,
$$

and consequently $\varphi(t) \rightarrow \varphi(0)$ for $t \downarrow 0$. This shows the $v$-hemicontinuity of $F$.

The next lemma is known as Minty lemma or Linearization lemma. It states an equivalent formulation for problem (2.1), which will turn out to be very useful in the next sections.

Lemma 4.1 (Minty, [53, 98]). Besides assumption (A), let $F: X \rightarrow \mathrm{L}(X, Y)$ be a $K$-monotone and vhemicontinuous mapping. Then, an element $x \in C$ is a solution of the vector variational inequality (2.1), that is, satisfies

$$
\langle F x, y-x\rangle_{Y} \notin-\operatorname{int} K, \quad \text { for every } \quad y \in C,
$$

if and only if it satisfies

$$
\langle F y, y-x\rangle_{Y} \notin-\operatorname{int} K, \quad \text { for every } \quad y \in C .
$$

Proof. Let $x \in C$ be a solution of problem (2.1). Since $F$ is $K$-monotone, we have

$$
\langle F x-F y, x-y\rangle_{Y} \in K, \quad \text { for every } y \in C .
$$

By adding the relations (2.1) and (4.2), we conclude that $x$ is a solution of problem (4.1); see Proposition 2.5 (iv). 
Conversely, let $x \in C$ be a solution of problem (4.1). Inserting the element $x+t(z-x) \in C$, where $z \in C$ and $0<t<1$ is sufficient small, we obtain

$$
t\langle F(x+t(z-x)), z-x\rangle_{Y} \notin-\operatorname{int} K .
$$

Multiplying the above inequality by $t^{-1}$ and passing to the limit $t \downarrow 0$ shows that $x \in C$ solves problem (2.1); compare Proposition 2.5 (v). The proof is complete.

Remark 4.2. (i) Under the assumptions of Lemma 4.1, it holds that

$$
\operatorname{Sol}(\mathrm{VVI})=\left\{x \in C \mid\langle F y, x-y\rangle_{Y} \notin-\operatorname{int} K, \text { for every } y \in C\right\} .
$$

This relation will turn out to be very useful in the next sections. See, for example, Theorem 5.1 and Theorem 5.15 in this paper.

(ii) Problem (4.1) is frequently called Minty vector variational inequality; see [98]. Further, Lemma 4.1 is the vector analogue of Lemma 1.5 in [144, Chapter III]. For a kind of symmetry, problem (4.1) is frequently called Stampacchia vector variational inequality; see, for example, [12].

Example 4.2. As a consequence of Lemma 4.1 and Example 4.1, the vector variational inequality (2.3) is equivalent to the following problem: find an element $x \in \mathbb{R}^{l}$ such that

$$
\left(\begin{array}{c}
\left\langle y-a^{1}, y-x\right\rangle \\
\vdots \\
\left\langle y-a^{k}, y-x\right\rangle
\end{array}\right) \notin-\operatorname{int} \mathbb{R}_{\geq}^{k}, \quad \text { for every } \quad y \in \mathbb{R}^{l} .
$$

The following proposition gives necessary conditions for the closedness and compactness of the solution set of the vector variational inequality (2.1).

Proposition 4.1. Besides assumption (A), let $F: X \rightarrow \mathrm{L}(X, Y)$ be a given mapping.

(i) Assume that $F$ is $K$-monotone and v-hemicontinuous. Then, the solution set of problem (2.1) is closed. If $C$ is compact in addition, then the solution set of problem (2.1) is compact.

(ii) Assume that $F$ is continuous. Then, the solution set of problem (2.1) is closed. If C is compact in addition, then the solution set of problem (2.1) is compact.

Proof. (i). Let $\left\{x_{n}\right\}$ be a sequence in Sol(VVI) with $x_{n} \rightarrow x$. By (4.3), it follows that

$$
\left\langle F y, x_{n}-y\right\rangle_{Y} \notin-\operatorname{int} K, \quad \text { for every } \quad y \in C .
$$

Passing to the limit and using the fact that the set $Y \backslash(-\operatorname{int} K)$ is closed in $Y$, we deduce $x \in \operatorname{Sol}(\mathrm{VVI})$. In other words, the solution set of problem (2.1) is closed. Since Sol(VVI) $\subseteq C$, the solution set is compact provided that $C$ is compact.

(ii). Let $\left\{x_{n}\right\}$ be a sequence in Sol(VVI) with $x_{n} \rightarrow x$. Note that for every $y \in X$

$$
\left\langle F x_{n}, y-x_{n}\right\rangle_{Y} \rightarrow\langle F x, y-x\rangle_{Y}
$$

see Proposition 2.4 (vi). Since $Y \backslash(-\operatorname{int} K)$ is a closed subset of $Y$, we conclude $x \in \operatorname{Sol}(\mathrm{VVI})$. The second part follows similar to the previous step. The proof is complete.

Remark 4.3. (i) In [79], Hu and Fang investigate conditions for the non-emptyness and compactness of the solution set of pseudomonotone vector variational inequalities by using the concept of asymptotical cones. The authors in [84] use linear scalarization to characterize the compactness of the solution set of generalized vector variational inequalities.

(ii) It should be noted that the solution set of problem (2.3) is compact, although the constraining set is unbounded. 
Since int $K \subseteq K \backslash\{0\}$, it is clear that we have

$$
\left\{x \in C \mid\langle F x, y-x\rangle_{Y} \notin-K \backslash\{0\}\right\} \subseteq \operatorname{Sol}(\mathrm{VVI}),
$$

but the converse inclusion does not hold in general; see, [155, Counterexample]. However, under additional assumptions for $C$ and $F$, a positive answer can be given for the finite-dimensional case. We therefore need the following definition.

Definition 4.2 ([155, Definition 3]). A non-empty subset $E \subseteq \mathbb{R}^{l}$ is said to be a strictly convex body if $\operatorname{int} E \neq \emptyset$ and for every $x, y \in E$ with $x \neq y$, it holds that $\{t x+(1-t) y \mid t \in(0,1)\} \subseteq \operatorname{int} E$.

Yen and Lee [155] provide the following answer for the finite-dimensional case.

Theorem 4.1 ([155, Theorem 2]). Besides assumption (A), let $C \subseteq \mathbb{R}^{l}$ be a strictly convex body and assume that for every $x \in C$, the mapping $\langle F x, \cdot\rangle_{\mathbb{R}^{k}}: \mathbb{R}^{l} \rightarrow \mathbb{R}^{k}$ is surjective. Then it holds

$$
\operatorname{Sol}\left(\mathrm{VVI}^{\prime}\right)=\left\{x \in C \mid\langle F x, y-x\rangle_{\mathbb{R}^{k}} \notin-\mathbb{R}_{\geq}^{k} \backslash\{0\}\right\} .
$$

Proof. Suppose to the contrary that (4.4) does not hold. Then we can find an element $x \in \operatorname{Sol}\left(\mathrm{VVI}^{\prime}\right)$ such that $x \notin\left\{x \in C \mid\langle F x, y-x\rangle_{\mathbb{R}^{k}} \notin-\mathbb{R}_{\geq}^{k} \backslash\{0\}\right\}$. Consequently, there is an element $y \in C$ such that

$$
\langle F x, y-x\rangle_{\mathbb{R}^{k}} \in-\mathbb{R}_{\geq}^{k} \backslash\{0\} .
$$

Let us define $x_{t}:=(1-t) x+t y$ for $t \in(0,1)$. Note that $x_{t} \in \operatorname{int} C$. Multiplying the above inequality by $t$, we conclude

$$
\left\langle F x, x_{t}-x\right\rangle_{\mathbb{R}^{k}} \in-\mathbb{R}_{\geq}^{k} \backslash\{0\} .
$$

Now let $\varepsilon>0$ be such that $B\left(x_{t}, \varepsilon\right) \subseteq C$, where $B\left(x_{t}, \varepsilon\right)$ denotes the closed ball centered at $x_{t}$ with radius $\varepsilon$. Since by assumption $\langle F x, \cdot\rangle_{\mathbb{R}^{k}}$ is linear and surjective, Banach's open mapping theorem yields that the mapping is open; see [27, Theorem 2.6]. Consequently, the image $\left\langle F x, B\left(x_{t}, \varepsilon\right)-x\right\rangle_{\mathbb{R}^{k}}:=\left\{\langle F x, y-x\rangle_{\mathbb{R}^{k}} \mid\right.$ $\left.y \in B\left(x_{t}, \varepsilon\right)\right\}$ must be a neighborhood of $z_{t}:=\left\langle F x, x_{t}-x\right\rangle_{\mathbb{R}^{k}}$. Now let $\rho>0$ be, such that $B\left(z_{t}, \rho\right) \subseteq$ $\left\langle F x, B\left(x_{t}, \varepsilon\right)-x\right\rangle_{\mathbb{R}^{k}}$. Consequently, we deduce from (4.5) that it holds $B\left(z_{t}, \rho\right) \cap\left(-\operatorname{int} \mathbb{R}^{k} \backslash\{0\}\right) \neq \emptyset$; compare [179, Corollary 6.3.2]. But this implies that we can find an element $\tilde{y} \in B\left(x_{t}, \varepsilon\right) \subseteq C$ with

$$
\langle F x, \tilde{y}-x\rangle_{\mathbb{R}^{k}} \in-\operatorname{int} \mathbb{R}^{k},
$$

which is impossible. The proof is complete.

4.2. Connectedness and semi-algebraicness. In this subsection, results ensuring that the solution set of the finite-dimensional vector variational inequality (2.2) is connected and semi-algebraic will be investigated. To proceed furthermore with our discussion, the following definition is needed.

Definition 4.3 ([184, Definition 2.5.2]). A subset $E$ of $\mathbb{R}^{l}$ is said to be connected if there are no two open, disjoint sets $O_{1}$ and $O_{2}$ in $\mathbb{R}^{l}$ with $O_{1} \cup O_{2}=E$ beside $E$ and $\emptyset$.

Remark 4.4. Convex sets are connected; see Proposition 2.5.8 in [184].

The next theorem is based on the following result.

Proposition 4.2 ([118, Theorem 3.1, Theorem 3.2]). Let $H: \mathbb{R}^{k} \rightrightarrows \mathbb{R}^{l}$ be an upper semicontinuous setvalued mapping and let $S \subseteq \mathbb{R}^{k}$ be a connected set. If for every $s \in S$, the set $H(s)$ is non-empty and connected, then the image

$$
H(S):=\bigcup_{s \in S} H(s)
$$

is connected. 
Remark 4.5. A set-valued mapping $H: \mathbb{R}^{k} \rightrightarrows \mathbb{R}^{l}$ is said to be upper semicontinuous at $x \in \mathbb{R}^{k}$ if for any open set $V$ in $\mathbb{R}^{l}$ containing $H(x)$, there exists an open neighborhood $U$ of $x$ in $\mathbb{R}^{k}$ such that $H(z) \subseteq V$ for all $z \in U$. $H$ is said to be upper semicontinuous if it is upper semicontinuous at every point in $\mathbb{R}^{k}$. Continuity results for set-valued mappings will we investigated in detail in Section 10. However, a handy characterization of upper semicontinuity is as follows; compare Lemma 10.1: Let $H: \mathbb{R}^{k} \rightrightarrows \mathbb{R}^{k}$ be a setvalued mapping such that there exists a compact subset $B$ of $\mathbb{R}^{l}$ with $H(x) \subseteq B$ for every $x \in \mathbb{R}^{k}$. Then, $H$ is upper semicontinuous at $x \in \mathbb{R}^{k}$ if and only if for any sequence $\left\{x_{n}\right\} \subseteq \mathbb{R}^{k}, x_{n} \rightarrow x$ and for any $y \in H(x)$, there is a sequence $\left\{y_{n}\right\}$ such that $y_{n} \in H\left(x_{n}\right)$ and $y_{n} \rightarrow y$.

The next theorem can be found in [35, Theorem 2.3] and [57, Theorem 1].

Theorem 4.2. Besides assumption (A), let $F: \mathbb{R}^{l} \rightarrow \mathrm{Mat}_{k \times l}(\mathbb{R})$ be a $\mathbb{R}_{>}^{k}$-monotone and continuous mapping. If $C$ is bounded in addition, then the solution set of the finite-dimensional vector variational inequality (2.2) is connected.

Proof. The proof of this theorem is based on Proposition 4.2. We therefore define a set-valued mapping $H: \mathbb{R}^{k} \rightrightarrows \mathbb{R}^{l}$ by

$$
H(s):=\operatorname{Sol}\left(\mathrm{VI}_{s}^{\prime}\right), \quad \text { for every } \quad s \in \mathbb{R}^{k} .
$$

Recall that $\operatorname{Sol}\left(\mathrm{VI}_{s}^{\prime}\right)$ denotes the solution set of the scalar problem (3.1) with respect to $s$. Note further that we have

$$
\operatorname{Sol}\left(\mathrm{VVI}^{\prime}\right)=\bigcup_{s \in S} H(s)
$$

where $S:=\left\{s \in \mathbb{R}_{\geq}^{k} \mid\|s\|=1\right\}$; see Proposition 3.1 and Remark 3.1. Since $F$ is $\mathbb{R}_{\geq}^{k}$-monotone and continuous and $C$ is non-empty, closed and compact, the set $H(s)$ is non-empty for every $s \in S$; see, for example, [144, Theorem 3.1]. It remains to show that $H$ is an upper semicontinuous set-valued mapping with connected values. The values of $H$ are trivially connected since they are convex; see Remark 4.4. Indeed, note that problem (3.1) with respect to $s \in \mathbb{R}^{k}$ is equivalent to finding an element $x \in C$ such that

$$
\left\langle\sum_{j=1}^{k} s_{j} F_{j} y, y-x\right\rangle \geq 0, \quad \text { for every } \quad y \in C
$$

compare Lemma 4.1 and Remark 4.2. Thus, the convexity of $H(s)$ follows. Now assume to the contrary that $H$ is not upper semicontinuous at a point $s \in S$. Then we can find an open set $V$ in $\mathbb{R}^{l}$ with $H(s) \subseteq V$ such that for any open neighborhood $U$ of $s$, it holds $H(\tilde{s}) \subseteq V$ for all $\tilde{s} \in U$. This implies that there exists a sequence $\left\{s_{n}\right\}$ in $S$ with $s_{n} \rightarrow s$ such that for each index $n$, we can find $x_{n} \in H\left(s_{n}\right)$ with $x_{n} \notin V$. Consequently, since $x_{n}$ solves the scalar problem (3.1) with respect to $s_{n}$, we have $x_{n} \in C$ and

$$
\left\langle\sum_{j=1}^{k} s_{j}^{n} F_{j} x_{n}, y-x_{n}\right\rangle \geq 0, \quad \text { for every } y \in C,
$$

where $s_{n}=\left(s_{1}^{n}, \ldots, s_{k}^{n}\right)^{\top}$. Since $\left\{x_{n}\right\}$ lies in the compact set $C$, there is a subsequence, again denoted by $\left\{x_{n}\right\}$, such that $x_{n} \rightarrow x$. Now passing in the above inequality to the limit, we conclude

$$
\left\langle\sum_{j=1}^{k} s_{j} F_{j} x, y-x\right\rangle \geq 0, \quad \text { for every } y \in C .
$$

In other words, $x \in H(s)$. However, this is impossible since $x \notin V$ but $H(s) \subseteq V$. Therefore, $H$ is upper semicontinuous, which completes the proof.

Remark 4.6. The set-valued mapping $H: \mathbb{R}^{k} \rightrightarrows \mathbb{R}^{l}$, given by relation (4.6), is frequently called base multifunction associated to problem (2.2); see Section 2 in [120]. 
Definition 4.4 ([24, Definition 2.1.4]). A subset $E$ of $\mathbb{R}^{l}$ is called semi-algebraic if it is of the form

$$
E=\bigcup_{i=1}^{s} \bigcap_{j=1}^{r_{i}}\left\{x \in \mathbb{R}^{l} \mid f_{i j}(x) \sim_{i j} 0\right\}
$$

where $s, r_{1}, \ldots, r_{s} \in \mathbb{N}, f_{i j} \in \mathscr{P}\left(\mathbb{R}^{l}\right)$ and $\sim_{i j} \in\{<,=\}$ for $i=1, \ldots, s$ and $j=1, \ldots, r_{i}$. Here, $\mathscr{P}\left(\mathbb{R}^{l}\right)$ denotes the ring of real polynomials in $l$ variables.

Remark 4.7. (i) It should be noted that semi-algebraic subsets of $\mathbb{R}^{l}$ form the smallest family of subsets containing all sets of the form $\left\{x \in \mathbb{R}^{l} \mid f(x)>0\right\}$, where $f \in \mathscr{P}\left(\mathbb{R}^{l}\right)$, and closed under taking finite intersections, finite unions and complements; see Section 2 in [24].

(ii) Open balls, closed balls and spheres in $\mathbb{R}^{l}$, where $\mathbb{R}^{l}$ is equipped with the Euclidean topology, are semi-algebraic sets. Numerous examples and properties of semi-algebraic sets can be found in [21,24] and Example 4.3 of this paper.

The following theorem states that the solution set of so-called polynomial vector variational inequalities [125] is semi-algebraic.

Theorem 4.3 ([125, Theorem 3.1]). Besides assumption (A), let $F: \mathbb{R}^{l} \rightarrow \mathrm{Mat}_{k \times l}(\mathbb{R})$ be a given mapping and assume that the following conditions hold:

(i) All components of $F$ are polynomials.

(ii) The constraining set $C$ is a polyhedral set, that is, there are $p \in \mathbb{N}, A \in \operatorname{Mat}_{p \times l}(\mathbb{R})$ and $b \in \mathbb{R}^{p}$ such that $C=\left\{x \in \mathbb{R}^{l} \mid b-A x \in \mathbb{R}_{\geq}^{p}\right\}$.

Then it holds that the solution set of the finite-dimensional variational inequality (2.2) is a semi-algebraic subset of $\mathbb{R}^{l}$.

Proof. Let $J_{p}:=\{1, \ldots, p\}$ and define for every $J \subseteq J_{p}$ the so-called pseudo-face

$$
C_{J}:=\left\{x \in \mathbb{R}^{l} \mid \sum_{j=1}^{l} a_{i j} x_{j}=b_{i}, \text { for every } i \in J, \sum_{j=1}^{l} a_{i j} x_{j}<b_{i}, \text { for every } i \notin J\right\}
$$

of $C$. It is easily seen that $C=\bigcup_{J \subseteq J_{p}} C_{J}$. Thus, we can rewrite the solution set of problem (2.2) as

$$
\operatorname{Sol}\left(\mathrm{VVI}^{\prime}\right)=\bigcup_{J \subseteq J_{p}} \operatorname{Sol}\left(\mathrm{VVI}^{\prime}\right) \cap C_{J}
$$

Since a finite union of semi-algebraic sets remains semi-algebraic, the proof is completed if we show that every set $\operatorname{Sol}\left(\mathrm{VVI}^{\prime}\right) \cap C_{J}$, where $J \subseteq J_{p}$, is semi-algebraic; compare [21]. However, the main idea is to use the reformulation $\operatorname{Sol}\left(\mathrm{VVI}^{\prime}\right) \cap C_{J}=\operatorname{Proj}\left(\Omega_{J}\right)$, where Proj : $\mathbb{R}^{l} \times \mathbb{R}^{k} \rightarrow \mathbb{R}^{l}$ is the natural projection on the space of the first $l$ components and $\Omega_{J} \subseteq \mathbb{R}^{l} \times \mathbb{R}^{k}$ is a semi-algebraic set, which is constructed using Farka's lemma. Since Proj preserves semi-algebraicness, see Theorem 2.2.1 in [24], the semialgebraicness of Sol( $\left.\mathrm{VVI}^{\prime}\right)$ follows. The proof is complete.

Remark 4.8. In [119], Hieu studies the connectedness and boundedness structure of the solution set of a finite-dimensional vector variational inequality by using the famous Tarski-Seidenberg Theorem in the second form; see Theorem 2.2.1 in [24]. In [93, Section 3], Yu et al. investigate the connectedness of the solution set of vector variational inequalities. Similar results, using linear scalarization methods, can be found in $[35,57,78]$. The authors in [155] study the path-connectedness and connectedness of finitedimensional vector variational inequalities with lipschitz continuous and strongly monotone mappings. Scalarization methods and several properties of semi-algebraic sets are also used in [125] to prove that polynomial vector variational inequalities have finitely many connected components. Consequences of such results in the field of multi-objective optimization are discussed as well. In [120], Hieu establishes several upper and lower estimates for the maximal number of the connected components of the solution set of a monotone affine vector variational inequality. 
Example 4.3. Let us come back to Example 2.2. Evidently, the assumptions of Theorem 4.3 hold, that is, the solution set $\mathrm{S}$ of problem (2.3) is a semi-algebraic set. Note that the whole space $\mathbb{R}^{l}$ is trivially a polyhedral set. In the previous section, we have shown that $\mathrm{S}$ is equivalent to the convex hull of $a^{1}, \ldots, a^{k}$. In what follows, we again confirm that $\mathrm{S}$ is semi-algebraic, where we let $l=2$ and $k=5$. In order to show that $\mathrm{S}=\operatorname{conv}\left\{a^{1}, \ldots, a^{5}\right\}$ is a semi-algebraic set in $\mathbb{R}^{2}$, consider the following list of sets:

$$
\begin{array}{lll}
A_{11}=\left\{x \in \mathbb{R}^{2} \mid x_{1}-a_{1}^{1}>0\right\}, & A_{12}=\left\{x \in \mathbb{R}^{2} \mid a_{1}^{4}-x_{1}>0\right\}, \quad A_{13}=\left\{x \in \mathbb{R}^{2} \mid x_{2}-a_{2}^{1}>0\right\}, \\
A_{14}=\left\{x \in \mathbb{R}^{2} \mid a_{2}^{2}-x_{2}>0\right\}, & \\
A_{21}=\left\{x \in \mathbb{R}^{2} \mid x_{1}-a_{1}^{1}=0\right\}, & A_{22}=\left\{x \in \mathbb{R}^{2} \mid x_{2}-a_{2}^{1}>0\right\}, \quad A_{23}=\left\{x \in \mathbb{R}^{2} \mid a_{2}^{2}-x_{2}>0\right\}, \\
A_{31}=\left\{x \in \mathbb{R}^{2} \mid x_{2}-a_{2}^{2}=0\right\}, & A_{32}=\left\{x \in \mathbb{R}^{2} \mid x_{1}-a_{1}^{2}>0\right\}, \quad A_{33}=\left\{x \in \mathbb{R}^{2} \mid a_{1}^{3}-x_{1}>0\right\}, \\
A_{41}=\left\{x \in \mathbb{R}^{2} \mid x_{1}-a_{1}^{3}=0\right\}, & A_{42}=\left\{x \in \mathbb{R}^{2} \mid x_{2}-a_{2}^{3}>0\right\}, \quad A_{43}=\left\{x \in \mathbb{R}^{2} \mid a_{2}^{4}-x_{2}>0\right\}, \\
A_{51}=\left\{x \in \mathbb{R}^{2} \mid x_{2}-a_{2}^{1}=0\right\}, & A_{52}=\left\{x \in \mathbb{R}^{2} \mid x_{1}-a_{1}^{1}>0\right\}, \quad A_{53}=\left\{x \in \mathbb{R}^{2} \mid a_{1}^{4}-x_{1}>0\right\}, \\
A_{61}=\left\{x \in \mathbb{R}^{2} \mid x_{1}-a_{1}^{1}=0\right\}, & A_{62}=\left\{x \in \mathbb{R}^{2} \mid x_{2}-a_{2}^{1}=0\right\}, \\
A_{71}=\left\{x \in \mathbb{R}^{2} \mid x_{1}-a_{1}^{2}=0\right\}, & A_{72}=\left\{x \in \mathbb{R}^{2} \mid x_{2}-a_{2}^{2}=0\right\}, \\
A_{81}=\left\{x \in \mathbb{R}^{2} \mid x_{1}-a_{1}^{3}=0\right\}, & A_{82}=\left\{x \in \mathbb{R}^{2} \mid x_{2}-a_{2}^{3}=0\right\}, \\
A_{91}=\left\{x \in \mathbb{R}^{2} \mid x_{1}-a_{1}^{4}=0\right\}, & A_{92}=\left\{x \in \mathbb{R}^{2} \mid x_{2}-a_{2}^{4}=0\right\} .
\end{array}
$$

Then it holds

$$
\mathrm{S}=\operatorname{conv}\left\{a^{1}, \ldots, a^{5}\right\}=\bigcup_{i=1}^{9} \bigcap_{j=1}^{r_{i}} A_{i j},
$$

where $r_{1}=4, r_{2}=\ldots=r_{5}=3$ and $r_{6}=\ldots=r_{9}=2$, which again confirms that the solution set of problem (2.3) is semi-algebraic.

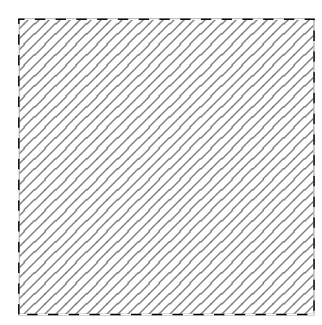

(A) $\bigcap_{j=1}^{4} A_{1 j}$

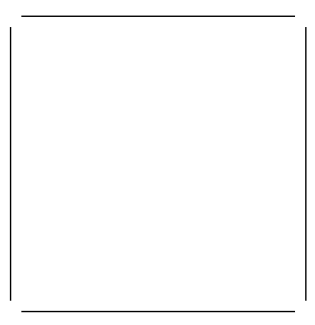

(в) $\bigcup_{i=2}^{5} \bigcap_{j=1}^{3} A_{i j}$
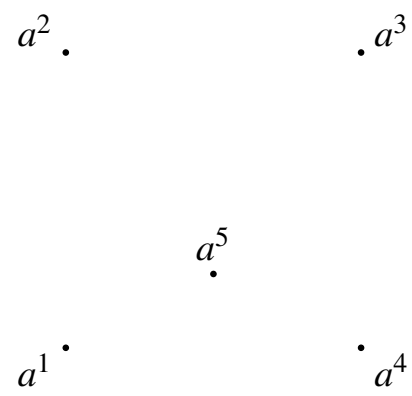

(C) $\bigcup_{i=6}^{9} \bigcap_{j=1}^{2} A_{i j}$

FIGURE 7. Illustration of certain unions and intersections of sets $A_{i j}$.

It should be notated that, although the constraining set in problem (2.3) is unbounded, its solution set is convex and therefore connected.

\section{EXISTENCE RESULTS}

This section is devoted to the study of existence results for vector variational inequality (2.1). Within the last decades, several existence results for vector variational inequalities of type (2.1) and extensions of it have been published in the literature. However, a careful study of all results shows that only a handful of different techniques are used; compare, for example, [7, 8, 12, 13, 31, 40, 53, 81, 82, 101, $111,115,133,136,138,140,141,146,163,165,176,186,193,194]$ and the references therein. We will therefore focus on the basic vector variational inequality (2.1) only since all results and methods for generalized vector variational inequalities are based on the results for problem (2.1). In order to ensure the existence of solutions, a so-called coercivity (or boundedness) condition of the data of the vector 
variational inequality is required - in this setting, either for the mapping $F$ or for the constraining set $C$. In general, this condition is crucial because otherwise, the existence of solutions cannot be guaranteed. However, in absence of coercivity conditions, one can replace problem (2.1) by a family of so-called regularized vector variational inequalities. Even though the data of (2.1) do not enjoy any coercivity condition, one can show that the regularized problems attain a solution. It turns out that the limit point of any sequence of regularized solutions solves problem (2.1). The regularization therefore allows one to derive existence results for non-coercive vector variational inequalities. In what follows, the main tools for deriving existing results for problem (2.1) are the Fan-KKM lemma, the Hartmann-Stampacchia theorem, Browder's fixed-point theorem, Fan's section lemma and Brouwer's fixed-point theorem, which we will recall in this section.

5.1. Existence results with monotonicity. This section is devoted to existence results for problem (2.1), where the mapping $F: X \rightarrow \mathrm{L}(X, Y)$ is assumed to be $K$-monotone; compare Definition 4.1. Let us recall the famous Fan-KKM lemma by Fan, Knaster, Kuratowski and Mazurkiewicz, which will turn out to be a crucial tool in the following.

Lemma 5.1 ([76, Lemma 1], Fan-KKM). Let C be a non-empty subset of the topological vector space $X$ and let $G: C \rightrightarrows X$ be a set-valued mapping with non-empty values. Then it holds

$$
\bigcap_{y \in C} G(y) \neq \emptyset
$$

provided that $G$ satisfies the following properties:

(i) For any $k \in \mathbb{N}$ and any finite subset $\left\{x_{1}, \ldots, x_{k}\right\} \subseteq C$ it holds that $\operatorname{conv}\left\{x_{1}, \ldots, x_{k}\right\} \subseteq \bigcup_{j=1}^{k} G\left(x_{j}\right)$.

(ii) For every $y \in C, G(y)$ is closed in $X$.

(iii) $G\left(y_{0}\right)$ is compact in $X$ for some $y_{0} \in C$.

Remark 5.1. (i) An excellent overview about applications of the Fan-KKM lemma in the field of setvalued fixed-point results, minimax equalities and inequalities and variational inequalities can be found in $[152,173]$.

(ii) A set-valued mapping, satisfying condition (i) in Lemma 5.1, is said to be a KKM-mapping, see [12, Definition 1.48].

(iii) It is easily seen that the set-valued mapping $G: C \rightrightarrows X$ is a KKM-mapping if it holds $y \in G(y)$ and $X \backslash G^{-1}(y)$ is convex for every $y \in C$.

The next theorem provides a first existence result for the vector variational inequality (2.1). It will turn out that the technique which is used, will also be applied in several other existence results.

Theorem 5.1 ([53, Theorem 2.1]). Besides assumption (A), let $F: X \rightarrow \mathrm{L}(X, Y)$ be a $K$-monotone and $v$-hemicontinuous mapping. If $C$ is bounded in addition, then the vector variational inequality (2.1) has a solution.

Proof. The proof of this theorem is based on the Fan-KKM lemma. We therefore introduce set-valued mappings $G, G^{\prime}: C \rightrightarrows X$ by

$$
G(y):=\left\{x \in C \mid\langle F x, y-x\rangle_{Y} \notin-\operatorname{int} K\right\} \quad \text { and } \quad G^{\prime}(y):=\left\{x \in C \mid\langle F y, y-x\rangle_{Y} \notin-\operatorname{int} K\right\},
$$

for every $y \in C$. Note that the values of $G$ and $G^{\prime}$ are non-empty since $y \in G(y)$ and $y \in G^{\prime}(y)$ for every $y \in C$. Since the mapping $F$ is assumed to be $K$-monotone and $v$-hemicontinuous, it is easily seen that it holds that

$$
\bigcap_{y \in C} G(y)=\operatorname{Sol}(\mathrm{VVI})=\bigcap_{y \in C} G^{\prime}(y)
$$


see Remark 4.2. Consequently, the proof is complete by showing that one of the intersections in (5.1) is non-empty. Let us show that the assumptions of Lemma 5.1 are satisfied.

(I). Let us show that $G$ is a KKM-mapping. Indeed, let $k \in \mathbb{N}$ and $\left\{x_{1}, \ldots, x_{k}\right\} \subseteq C$. Suppose to the contrary that

$$
\operatorname{conv}\left\{x_{1}, \ldots, x_{k}\right\} \nsubseteq \bigcup_{j=1}^{k} G\left(x_{j}\right) .
$$

Then there are real numbers $\lambda_{1}, \ldots, \lambda_{k}$ with $\sum_{j=1}^{k} \lambda_{j}=1$ and $\lambda_{j} \geq 0$ for $j=1, \ldots, k$ such that

$$
\sum_{j=1}^{k} \lambda_{j} x_{j} \notin \bigcup_{j=1}^{k} G\left(x_{j}\right) .
$$

In other words, it holds

$$
\left\langle F \bar{x}, x_{j}-\bar{x}\right\rangle_{Y} \in-\operatorname{int} K, \quad \text { for } \quad j=1, \ldots, k,
$$

where we define $\bar{x}:=\sum_{j=1}^{k} \lambda_{j} x_{j}$. Thus, multiplying every relation by $\lambda_{j}$ and summing them up, we have

$$
\sum_{j=1}^{k} \lambda_{j}\left\langle F \bar{x}, x_{j}\right\rangle_{Y} \in\langle F \bar{x}, \bar{x}\rangle_{Y}-\operatorname{int} K
$$

However, the left-hand side of this relation is equivalent to $\langle F \bar{x}, \bar{x}\rangle_{Y}$. The previous relation consequently states $0 \in \operatorname{int} K$. This is impossible since the cone $K$ is assumed to be proper; compare Remark 2.4. This shows that $G$ is a KKM-mapping.

(II). Let us show that $G^{\prime}$ is a KKM-mapping. Indeed, by the $K$-monotonicity of $F$, we have for every $y \in C$

$$
G(y) \subseteq G^{\prime}(y),
$$

compare the proof of Lemma 4.1. Consequently, $G^{\prime}$ is also a KKM-mapping.

(III). Let us show that $G^{\prime}$ has closed values. For this purpose, let $y \in C$ and let $\left\{x_{n}\right\} \subseteq G^{\prime}(y)$ be a sequence such that $x_{n} \rightarrow x$. Consequently,

$$
\left\langle F y, x_{n}-y\right\rangle_{Y} \notin-\operatorname{int} K \text {. }
$$

Using Proposition 2.4 and the fact that the set $Y \backslash(-\operatorname{int} K)$ is closed, passing in the above relation to the limit yields $\langle F y, x-y\rangle_{Y} \notin-\operatorname{int} K$. We have therefore shown that $x \in G^{\prime}(y)$. Since $y \in C$ was chosen arbitrarily, $G^{\prime}$ has closed values.

(IV). We now equip $X$ with the weak topology. Then $C$, as a closed, convex and bounded set in the reflexive Banach space $X$, is weakly compact. Therefore, for every $y \in C, G^{\prime}(y)$ is weakly compact as a weakly closed subset of $C$. We have finally shown that $G^{\prime}: C \rightrightarrows X$ is a KKM-mapping with closed and compact values with respect to the weak topology of $X$. Thus, by Lemma 5.1, we obtain

$$
\bigcap_{y \in C} G^{\prime}(y) \neq \emptyset
$$

that is, (5.1) is non-empty. In other words, the solution set of problem (2.1) is non-empty. The proof is complete.

Remark 5.2. (i) Evidently, the requirements for the constraining set $C$ in Theorem 5.1 can be replaced in the following way: $C$ is non-empty, convex and weakly compact; compare [146, Theorem 3.1]. Further, if the set $C$ is assumed to be compact, then the reflexivity of $X$ can be dropped.

(ii) It should be noted that part (I) in the proof of Theorem 5.1, that is, the proof that $G: C \rightrightarrows X$ is a KKM-mapping does not use any properties of $F$ and $C$. 
(iii) The Fan-KKM Lemma 5.1 cannot be applied to the establishment of the existence of vector variational inequalities of the following type: find an element $x \in C$ such that

$$
\langle F x, y-x\rangle_{Y} \notin-K \backslash\{0\}, \quad \text { for every } \quad y \in C .
$$

This is due to the fact that the corresponding set-valued mappings $G$ and $G^{\prime}$, compare the previous proof, do not have closed values in general.

(iv) Several other existence results for problem (2.1) and generalizations of it, based on the Fan-KKM lemma, can be found, for example, in $[3,14,59,80,82,93,112,123,133,135,136,138,139,145,165$, $176,186,188,194]$.

In the following theorem, the $K$-monotonicity of $F$ is replaced by a so-called L-condition. See [12, Chapter 5] for several other existence results based on the L-condition.

Theorem 5.2 ([189, Proposition 1]). Besides assumption (A), let $F: X \rightarrow \mathrm{L}(X, Y)$ be a v-hemicontinuous mapping, which satisfies the following L-condition: for any $k \in \mathbb{N}$, for any finite subset $\left\{x_{1}, \ldots, x_{k}\right\} \subseteq C$ and for all $\lambda_{1}, \ldots, \lambda_{k}$ with $\lambda_{j} \geq 0$ for $j=1, \ldots, k$ and $\sum_{j=1}^{k} \lambda_{j}=1$ it holds

$$
\sum_{j=1}^{k} \lambda_{j}\left\langle F x_{j}, x_{j}\right\rangle_{Y}-\sum_{j=1}^{k} \lambda_{j}\left\langle F x_{j}, \bar{x}\right\rangle_{Y} \in K,
$$

where $\bar{x}:=\sum_{j=1}^{k} \lambda_{j} x_{j}$. If in addition, $C$ is bounded, then the vector variational inequality (2.1) has a solution.

Proof. This proof is again based on the Fan-KKM lemma. Recall that in the proof of Theorem 5.1, the set-valued mapping $G^{\prime}: C \rightrightarrows X$ was defined by

$$
G^{\prime}(y):=\left\{x \in C \mid\langle F y, y-x\rangle_{Y} \notin-\operatorname{int} K\right\}, \quad \text { for every } \quad y \in C .
$$

Evidently, any element in the intersection

$$
\bigcap_{y \in C} G^{\prime}(y)
$$

is a solution of the Minty vector variational inequality (4.1). Further, since $F$ is assumed to be $v$ hemicontinuous, any solution of problem (4.1) is one of (2.1), see Lemma 4.1. It therefore remains to show that the requirements of Lemma 5.1 are satisfies in order to ensure that (5.3) is non-empty. A careful study of the proof of Theorem 5.1 shows that we have to adapt step (II) only; compare also the previous remark.

(I). Let us show that $G^{\prime}$ is a KKM-mapping. Indeed, let $k \in \mathbb{N}$ and $\left\{x_{1}, \ldots, x_{k}\right\} \subseteq C$. Suppose to the contrary that $\operatorname{conv}\left\{x_{1}, \ldots, x_{k}\right\}$ is not contained in $\bigcup_{j=1}^{k} G^{\prime}\left(x_{j}\right)$. Then there are $\lambda_{1}, \ldots, \lambda_{k}$ with $\sum_{j=1}^{k} \lambda_{j}=1$ and $\lambda_{j} \geq 0$ for $j=1, \ldots, k$ such that

$$
\sum_{j=1}^{k} \lambda_{j} x_{j} \notin \bigcup_{j=1}^{k} G^{\prime}\left(x_{j}\right) .
$$

In other words, it holds

$$
\left\langle F x_{j}, x_{j}-\bar{x}\right\rangle_{Y} \in-\operatorname{int} K, \quad \text { for } \quad j=1, \ldots, k,
$$

where we again define $\bar{x}:=\sum_{j=1}^{k} \lambda_{j} x_{j}$. Consequently, by multiplying every relation by $\lambda_{j}$ and summing them up, we obtain

$$
\sum_{j=1}^{k} \lambda_{j}\left\langle F x_{j}, \bar{x}\right\rangle_{Y}-\sum_{j=1}^{k} \lambda_{j}\left\langle F x_{j}, x_{j}\right\rangle_{Y} \in \operatorname{int} K
$$


Since $K$ is a convex and solid cone, it holds $K+\operatorname{int} K=\operatorname{int} K$; see [12, Proposition 2.7]. From (5.2) and (5.4), we therefore conclude that $0 \in \operatorname{int} K$. This is impossible; compare again Remark 2.4. Thus, $G^{\prime}$ is a KKM-mapping.

(II). Similar to the proof of Theorem 5.1, see part (III) and (IV), follows that the values of $G^{\prime}$ are nonempty, closed and compact with respect to the weak topology of $X$. Therefore, by applying Lemma 5.1, the intersection (5.3) is non-empty. In other words, the Minty vector variational inequality (4.1) has a solution. Since $F$ is $v$-hemicontinuous, it follows that the vector variational inequality (2.1) has a solution; see Lemma 4.1. The proof is complete.

In what follows, we will need the following definition, which can be found in [189].

Definition 5.1. Besides assumption (A), let $F: X \rightarrow \mathrm{L}(X, Y)$ be a given mapping. $F$ is said to be $v$ coercive if there exists a non-empty and compact subset $B \subseteq X$ and an element $y_{0} \in B \cap C$ such that

$$
\left\langle F y, y_{0}-y\right\rangle_{Y} \in \operatorname{int} K, \quad \text { for every } \quad y \in C \backslash B .
$$

Remark 5.3. (i) It is easily seen that $F$ is $v$-coercive with $B=C$ provided that $C$ is a compact set.

(ii) See [12, Definition 5.7] for several other $v$-coercivity conditions, which are related to the previous definition.

In the next result, the boundedness of $C$ in Theorem 5.1 is replaced by the $v$-coercivity of $F$.

Theorem 5.3 ([189, Theorem 1]). Besides assumption (A), let $F: X \rightarrow \mathrm{L}(X, Y)$ be a $K$-monotone, $v$ hemicontinuous and $v$-coercive mapping. Then, the vector variational inequality (2.1) has a solution.

Proof. We follow the proof of Theorem 5.1. In the previous proof, using the $K$-monotonicity and $v$ hemicontinuity of $F$, we have shown that the set-valued mapping $G^{\prime}: C \rightrightarrows X$, defined by

$$
G^{\prime}(y):=\left\{x \in C \mid\langle F y, y-x\rangle_{Y} \notin-\operatorname{int} K\right\},
$$

for every $y \in C$, is a KKM-mapping with non-empty and closed values; compare the steps (I), (II) and (III). In order to apply Lemma 5.1 and to prove that (5.1) is non-empty, it remains to show that there is an element $y_{0} \in C$ such that $G^{\prime}\left(y_{0}\right)$ is compact in $X$. Indeed, by the $v$-coercivity of $F$, there exists a non-empty and compact subset $B \subseteq X$ and an element $y_{0} \in B \cap C$ such that (5.5) holds. We consequently obtain

$$
G^{\prime}\left(y_{0}\right) \subseteq B,
$$

implying that $G^{\prime}\left(y_{0}\right)$ is compact as a closed subset of the compact set $B$. Note that in this setting $X$ is endowed with the usual norm topology. Finally, the Fan-KKM Lemma 5.1 ensures that the intersection in (5.1) is non-empty, implying that the vector variational inequality (2.1) has a solution.

Remark 5.4. (i) The reflexivity of $X$ can be dropped in Theorem 5.3.

(ii) In comparison to Theorem 5.1, the $v$-coercivity condition ensures the compactness of the set $G^{\prime}\left(y_{0}\right)$ only, while the boundedness of $C$ implies the weak compactness of all sets $G^{\prime}(y), y \in C$.

(iii) The results in $[13,138,140,165,186]$ use a similar technique as proposed in the previous theorem.

Recall that for $x_{0} \in X$ and $r>0, B\left(x_{0}, r\right):=\left\{x \in X \mid\left\|x-x_{0}\right\|_{X} \leq r\right\}$ denotes the closed ball with center $x_{0}$ and radius $r>0$ in $X$, while bd $B\left(x_{0}, r\right)$ denotes its boundary.

The following result uses a truncating argument.

Theorem 5.4 ([146, Theorem 3.3]). Besides assumption (A), let $F: X \rightarrow \mathrm{L}(X, Y)$ be a $K$-monotone and $v$-hemicontinuous mapping. If it holds that $0 \in C$ and there exists an element $y_{0} \in C$ and a number $d>0$ such that

$$
\left\langle F x, y_{0}-x\right\rangle_{Y} \in-\operatorname{int} K,
$$

for every $x \in C$ such that $\left\|y_{0}-x\right\|_{X}>d$, then the vector variational inequality (2.1) has a solution. 
Proof. We define $C_{r}:=C \cap B(0, r)$ for every $r>0$. Since $C_{r}$ is a non-empty, closed, convex and bounded subset of $X$ and $F$ is a $K$-monotone and $v$-hemicontinuous mapping, Theorem 5.1 implies that the following truncated vector variational inequality has a solution: find an element $x_{r} \in C_{r}$ such that

$$
\left\langle F x_{r}, y-x_{r}\right\rangle_{Y} \notin-\operatorname{int} K, \quad \text { for every } \quad y \in C_{r} .
$$

The proof is complete if we can show that (5.7) holds for all elements in $C$.

(I). Let us show that the corresponding sequence $\left\{x_{r}\right\}$ of solutions is bounded. Indeed, if not, then we can find an index $r>0$ large enough so that $\left\|y_{0}\right\|_{X} \leq r$ and $\left\|y_{0}-x_{r}\right\|_{X}>d$, where $y_{0} \in C$ and $d>0$ are given by the coercivity assumption of this theorem. By (5.6), it follows

$$
\left\langle F x_{r}, y_{0}-x_{r}\right\rangle_{Y} \in-\operatorname{int} K
$$

which contradicts (5.7). This shows that the sequence of solutions is bounded.

(II). Let us show that (5.7) holds for all elements in $C$. By step (I), there exists some $r>0$ such that $\left\|x_{r}\right\|_{X}<r$. Inserting the element $(1-t) x_{r}+t w \in C_{r}$ in (5.7), where $w \in C$ and $t>0$ is chosen small enough, we have

$$
t\left\langle F x_{r}, w-x_{r}\right\rangle_{Y} \notin-\operatorname{int} K \text {. }
$$

Recall that $w \in C$ was chosen arbitrarily. By applying Proposition 2.5 (v), we have finally shown that $x_{r} \in C$ satisfies

$$
\left\langle F x_{r}, w-x_{r}\right\rangle_{Y} \notin-\operatorname{int} K, \quad \text { for every } \quad w \in C .
$$

In other words, $x_{r} \in C$ is a solution of problem (2.1). The proof is complete.

Remark 5.5. (i) A similar method is used in [93, Theorem 2.2.3].

(ii) Note that the coercivity condition (5.6) can be equivalently stated as: there exists an element $y_{0} \in C$ and a number $d>0$ such that

$$
\left\langle F y, y_{0}-y\right\rangle_{Y} \in-\operatorname{int} K, \quad \text { for every } \quad y \in C \backslash B\left(y_{0}, d\right) .
$$

Therefore, if $X$ is a finite-dimensional Euclidean space, Theorem 5.3 recovers Theorem 5.4; see Theorem 2.1 .

The next result can be found in [130, Theorem 3.2], for instance. The same coercivity condition is also used in [31, 194].

Theorem 5.5. Besides assumption (A), let $F: X \rightarrow \mathrm{L}(X, Y)$ be a $K$-monotone and $v$-hemicontinuous mapping. If it holds that $0 \in C$ and there is a number $r>0$ such that

$$
\langle F x, x\rangle_{Y} \in \operatorname{int} K, \quad \text { for every } \quad x \in C \cap \operatorname{bd} B(0, r),
$$

then the vector variational inequality (2.1) has a solution.

Proof. The proof of this theorem follows similar to the one of Theorem 5.3. Let us define $C_{r}:=C \cap$ $B(0, r)$, where $r>0$ is given as in (5.8). Since $C_{r}$ is a non-empty, closed, convex and bounded subset of $X$ and $F$ is a $K$-monotone and $v$-hemicontinuous mapping, Theorem 5.1 implies that the following truncated vector variational inequality has a solution: find an element $x_{r} \in C_{r}$ such that

$$
\left\langle F x_{r}, y-x_{r}\right\rangle_{Y} \notin-\operatorname{int} K, \quad \text { for every } \quad y \in C_{r} .
$$

By inserting the zero element in (5.9), we obtain

$$
\left\langle F x_{r}, x_{r}\right\rangle_{Y} \notin \operatorname{int} K \text {. }
$$


The coercivity condition implies that $x_{r}$ does not belong to $C \cap \mathrm{bd} B(0, r)$, that is, we have in particular $x_{r} \in \operatorname{int} B(0, r)$. Inserting the element $(1-t) x_{r}+t w \in C_{r}$ in (5.7), where $w \in C$ and $t>0$ is chosen small enough, we have

$$
t\left\langle F x_{r}, w-x_{r}\right\rangle_{Y} \notin-\operatorname{int} K .
$$

Similar to the proof of Theorem 5.3, the previous inequality show that $x_{r} \in C$ is a solution of (2.1); see Proposition $2.5(\mathrm{v})$. The proof is complete.

Until now, we have investigated existence results, which aim to solve the vector variational inequality (2.1) in a direct way only. In contrast, the next existence result makes use of the fact that (scalar) variational inequalities are necessary for problem (2.1), provided that $s$ belongs the quasi-interior of $K^{*}$; compare Proposition 3.1. Recall that given $s \in Y^{*}$, the variational inequality (3.1) consists of finding an element $x \in C$ such that

$$
\langle s \circ F x, y-x\rangle_{X^{*}, X} \geq 0, \quad \text { for every } \quad y \in C .
$$

In order to show that problem (3.1) has a solution, we recall the following well-known theorem by Hartman and Stampacchia, which can be found in [144, Corollary 1.8].

Theorem 5.6 (Hartman-Stampacchia). Let $C$ be a non-empty, closed and convex subset of the real reflexive Banach space $X$. If $A: X \rightarrow X^{*}$ is a monotone and hemicontinuous operator, which is coercive in the sense that there is an element $x_{0} \in C$ such that

$$
\lim _{\|x\|_{X \rightarrow+\infty} \rightarrow \infty \in C} \frac{\left\langle A x-A x_{0}, x-x_{0}\right\rangle_{X^{*}, X}}{\left\|x-x_{0}\right\|_{X}}=+\infty,
$$

then the following variational inequality has a solution: find an element $x \in C$ such that

$$
\langle A x, y-x\rangle_{X^{*}, X} \geq 0, \quad \text { for every } y \in C .
$$

Remark 5.6. (i) It should be noted that the coercivity condition in Theorem 5.6 can be dropped provided the set $C$ is bounded in addition; see [144, Theorem 1.4].

(ii) If $A: X \rightarrow X^{*}$ is strictly monotone, then the solution of the above variational inequality is unique.

In order to formulate the next existence result, we need the following definition.

Definition 5.2 ([53, Section 2]). Besides assumption (A), assume that the quasi-interior of $K^{*}$ is nonempty and let $F: X \rightarrow \mathrm{L}(X, Y)$ be a given mapping. $F$ is said to be weakly coercive if there exist an element $x_{0} \in C$ and a functional $s \in$ qi $K^{*}$ such that

$$
\lim _{\|x\|_{X} \rightarrow+\infty} \frac{\left\langle s \circ F x-s \circ F x_{0}, x-x_{0}\right\rangle_{X^{*}, X}}{\left\|x-x_{0}\right\|_{X}}=+\infty .
$$

We have the following result, which is Theorem 2.1 in [53].

Theorem 5.7. Besides assumption (A), assume that the quasi-interior of $K^{*}$ is non-empty and let $F: X \rightarrow$ $\mathrm{L}(X, Y)$ be a $K$-monotone, $v$-hemicontinuous and weakly coercive mapping. Then, the vector variational inequality (2.1) has a solution.

Proof. The key idea of this proof is to show that the variational inequality (3.1) has a solution, where $s \in$ qi $K^{*}$ is given by the weak coercivity condition. Proposition 3.1 then implies that problem (2.1) has a solution since

$$
\operatorname{Sol}\left(\mathrm{VI}_{s}\right) \subseteq \operatorname{Sol}(\mathrm{VVI})
$$

Indeed, the solvability of problem (3.1) follows immediately from Theorem 5.6. Since $s: Y \rightarrow \mathbb{R}$ is linear and continuous in particular, the operator $s \circ F: X \rightarrow X^{*}$ is monotone and hemicontinuous. In addition 
to that, by (5.10), $s \circ F$ is coercive in the sense of Theorem 5.6. Thus, $\operatorname{Sol}\left(\mathrm{VI}_{s}\right) \neq \emptyset$ and the proof is complete.

Remark 5.7. Some other existence results, which are based on a scalarization technique, can be found in $[82,111,146,141,193]$. Further, an overview of linear scalarization for problem (3.2) can be found in Chapter 6 of [12].

Example 5.1. Let us show, using Theorem 5.7, that problem (2.3) has a solution. Since we have already shown in Example 4.1 that the mapping $F: \mathbb{R}^{l} \rightarrow \operatorname{Mat}_{k \times l}(\mathbb{R})$, given by (2.4), is $\mathbb{R}_{\geq}^{k}$-monotone and $v$ hemicontinuous, it remains to show that $F$ is weakly coercive. Indeed, define $x_{0}:=0$ and let $s \in \operatorname{int} \mathbb{R}_{\geq}^{k}$ be arbitrarily chosen. An easy calculation shows that relation (5.10) becomes

$$
\lim _{\|x\|_{2} \rightarrow+\infty} \frac{\left\langle s^{\top} F x-s^{\top} F x_{0}, x-x_{0}\right\rangle}{\left\|x-x_{0}\right\|_{2}}=\lim _{\|x\|_{2} \rightarrow+\infty}\|s\|_{1}\|x\|_{2}=+\infty .
$$

5.2. Existence results without monotonicity. This section is devoted to the study of existence results for problem (2.1), where, in comparison to the previous section, the $K$-monotonicity of $F$ is dropped.

The next result uses a strong continuity assumption of $F$, which we describe in the following definition.

Definition 5.3 ([115, Definition 2.4]). Besides assumption (A), let $F: X \rightarrow \mathrm{L}(X, Y)$ be a given mapping. $F$ is said to be strongly continuous if $x_{n} \rightarrow x$ in $X$ implies $F x_{n} \rightarrow F x$ in $\mathrm{L}(X, Y)$.

Remark 5.8. (i) Strongly continuous mappings are sometimes called completely continuous; see [41, Definition 3.13].

(ii) Clearly, if $X$ and $Y$ are finite-dimensional Euclidean spaces, then the notion of completely continuous and continuous mappings coincide; compare Proposition 2.4 (ii).

In order to formulate the next result, we need the following coercivity condition, which has been introduced in [115].

Definition 5.4. Besides assumption (A), let $F: X \rightarrow \mathrm{L}(X, Y)$ be a given mapping. $F$ is said to be $\kappa$ coercive if there exists a mapping $\kappa: X \rightarrow \mathbb{R}_{\geq}$and a functional $s \in K^{*} \backslash\{0\}$ such that

$$
\begin{aligned}
\langle s \circ F x, x\rangle_{X^{*}, X} & \geq\|s\|_{Y^{*}} \kappa(x), \quad \text { for every } \quad x \in C, \\
\lim _{\substack{\|x\|_{X \rightarrow+\infty} \rightarrow \infty \\
x \in C}} \frac{\kappa(x)}{\|x\|_{X}} & =+\infty .
\end{aligned}
$$

Theorem 5.8 ([115, Theorem 6]). Besides assumption (A), let $F: X \rightarrow \mathrm{L}(X, Y)$ be a strongly continuous and $\kappa$-coercive mapping. If it holds that $0 \in C$, then the vector variational inequality (2.1) has a solution.

Proof. The key idea of this proof is to show that the scalar variational inequality (3.1) has a solution, where $s \in K^{*} \backslash\{0\}$ is given by the $\kappa$-coercivity condition, since every solution of problem (3.1) is one of (2.1); see Proposition 3.1. For this purpose, let us define the set-valued mapping $G_{s}: C \rightrightarrows X$ by

$$
G_{s}(y):=\{x \in C \mid\langle s \circ F x, y-x\rangle \geq 0\}, \quad \text { for every } y \in C .
$$

Note that for every $y \in C$, it holds that $y \in G_{s}(y)$, that is, $G_{s}$ has non-empty values. Evidently, every element belonging to the intersection

$$
\bigcap_{y \in C} G_{s}(y)
$$

is a solution of (3.1). In order to prove that (5.11) is non-empty, we are going to show that the requirements of Lemma 5.1 are satisfied. 
(I). Let us show that $G_{s}$ is a KKM-mapping. Indeed, arguing by contradiction, let $k \in \mathbb{N}$ and $\left\{x_{1}, \ldots, x_{k}\right\} \subseteq$ $C$ and suppose to the contrary that

$$
\operatorname{conv}\left\{x_{1}, \ldots, x_{k}\right\} \nsubseteq \bigcup_{j=1}^{k} G_{s}\left(x_{j}\right) \text {. }
$$

Then there are real numbers $\lambda_{1}, \ldots, \lambda_{k}$ with $\sum_{j=1}^{k} \lambda_{j}=1$ and $\lambda_{j} \geq 0$ for $j=1, \ldots, k$ such that $\bar{x} \notin$ $\bigcup_{j=1}^{k} G_{s}\left(x_{j}\right)$, where $\bar{x}:=\sum_{j=1}^{k} \lambda_{j} x_{j}$. In other words, we have

$$
\left\langle s \circ F \bar{x}, x_{j}-\bar{x}\right\rangle_{X^{*}, X}<0, \quad \text { for } \quad j=1, \ldots, k .
$$

By multiplying every inequality by $\lambda_{j}$ and summing them up, we derive the contradiction

$$
\langle s \circ F \bar{x}, \bar{x}\rangle_{X^{*}, X}=\left\langle s \circ F \bar{x}, \sum_{j=1}^{k} \lambda_{j} x_{j}\right\rangle_{X^{*}, X}<\sum_{j=1}^{k} \lambda_{j}\langle s \circ F \bar{x}, \bar{x}\rangle_{X^{*}, X}=\langle s \circ F \bar{x}, \bar{x}\rangle_{X^{*}, X},
$$

showing that $G_{s}$ is a KKM-mapping.

(II). Let us show that $G_{s}$ has weakly closed values. Indeed, let $y \in C$ be arbitrarily chosen and consider a sequence $\left\{x_{n}\right\}$ in $G_{s}(y)$ with $x_{n} \rightarrow x$. Since $F$ is strongly continuous, we have $F x_{n} \rightarrow F x$ in $\mathrm{L}(X, Y)$ which implies

$$
\left\langle s \circ F x_{n}, y-x_{n}\right\rangle_{X^{*}, X} \rightarrow\langle s \circ F x, y-x\rangle_{X^{*}, X} ;
$$

see Proposition 2.4. Note that we have $x \in C$ since the set is assumed to be closed and convex. This shows $x \in G_{s}(y)$ and consequently $G_{s}$ has weakly closed values.

(III). Let us show that $G_{s}(0)$ is weakly compact. Since we have already shown that $G_{s}(0)$ is weakly closed, it remains to show that $G_{s}(0)$ is bounded. Assume by contradiction that $G_{s}(0)$ is unbounded. Then, we can find a sequence $\left\{x_{n}\right\}$ in $G_{s}(0)$ such that

$$
\left\|x_{n}\right\|_{X} \rightarrow+\infty \text {. }
$$

Consequently, using the $\kappa$-coercivity of $F$ and the fact that the sequence $\left\{x_{n}\right\}$ lies in $G_{s}(0)$, it follows that

$$
\|s\|_{Y^{*}} \frac{\kappa\left(x_{n}\right)}{\left\|x_{n}\right\|_{X}} \leq \frac{\left\langle s \circ F x_{n}, x_{n}\right\rangle_{X^{*}, X}}{\left\|x_{n}\right\|_{X}} \leq 0
$$

The above inequality leads to a contradiction when passing to the limit. Since $X$ is assumed to be reflexive, we have shown that $G_{s}(0)$ is weakly compact.

(IV). Finally, we are in position to apply Lemma 5.1, ensuring that the intersection (5.11) is non-empty. Consequently,

$$
\emptyset \neq \bigcap_{y \in C} G_{s}(y)=\operatorname{Sol}\left(\mathrm{VI}_{s}\right) \subseteq \operatorname{Sol}(\mathrm{VVI})
$$

see Proposition 3.1. We therefore have shown that the vector variational inequality (2.1) has a solution. The proof is complete.

The next example is Example 3.7 in [115].

Example 5.2. Let us come back to problem (2.3). In what follows, we will use Theorem 5.8 to show that the vector variational inequality (2.3) has a solution. Let us assume without any loss of generality that $a^{1}=0$ is the zero vector. Since the mapping $F$, given by (2.4), is obviously continuous, it remains to show that $F$ is $\kappa$-coercive. Indeed, let $s=\mathrm{e}_{1}$ and define $\kappa(x)=\|x\|_{2}^{2}$ for $x \in \mathbb{R}^{l}$. Then it holds

$$
\left\langle s^{\top} F x, x\right\rangle=\langle x, x\rangle=\|x\|_{2}^{2}=\|s\|_{2} \kappa(x), \quad \text { for every } \quad x \in \mathbb{R}^{l},
$$


and

$$
\lim _{\|x\|_{2} \rightarrow+\infty} \frac{\kappa(x)}{\|x\|_{2}}=\lim _{\|x\|_{2} \rightarrow+\infty}\|x\|_{2}=+\infty .
$$

Thus, $F$ is $\kappa$-coercive, that is, by applying Theorem 5.8, we deduce that problem (2.3) has a solution.

The next result shows that the $K$-monotonicity and $v$-hemicontinuity of $F$ in Theorem 5.1 can be replaced by the continuity of $F$. Again, the key idea of the proof in [53] is to make use of the Fan-KKM lemma.

Theorem 5.9 ([53, Theorem 2.2]). Besides assumption (A), let $F: X \rightarrow \mathrm{L}(X, Y)$ be a continuous mapping. If $C$ is bounded in addition, then the vector variational inequality (2.1) has a solution.

Proof. We follow the proof of Theorem 5.1. Recall that the set-valued mapping $G: C \rightrightarrows X$ was defined by

$$
G(y):=\left\{x \in C \mid\langle F x, y-x\rangle_{Y} \notin-\operatorname{int} K\right\}, \quad \text { for every } \quad y \in C .
$$

Since it holds

$$
\operatorname{Sol}(\mathrm{VVI})=\bigcap_{y \in C} G(y)
$$

we are going to show that the intersection in (5.12) is non-empty. In order to do so, we will use the Fan-KKM Lemma 5.1. From part (I) in the proof of Theorem 5.1 follows that $G$ is a KKM-mapping. It remains to show that $G$ has closed and compact values.

(I). Let us show that $G$ has closed values. Indeed, let $y \in C$ be arbitrarily chosen and let $\left\{x_{n}\right\} \subseteq G(y)$ be a sequence such that $x_{n} \rightarrow x$. Since $F x_{n} \rightarrow F x$ in $\mathrm{L}(X, Y)$, Proposition 2.4 (vi) implies that we have

$$
\left\langle F x_{n}, x_{n}-y\right\rangle_{Y} \rightarrow\langle F x, y-x\rangle_{Y} .
$$

Since $Y \backslash(-\operatorname{int} K)$ is closed, $x \in G(y)$. This shows that $G$ has closed values.

(II). We now equip $X$ with the weak topology. Then $C$, as a closed, convex and bounded set in the reflexive Banach space $X$, is weakly compact. Therefore, for every $y \in C, G(y)$ is weakly compact as a weakly closed subset of $C$. The Fan-KKM lemma finally ensures that the intersection in (5.12) is non-empty. The proof is complete.

Definition 5.5. Let $X$ be a topological space. A set-valued mapping $T: X \rightrightarrows X$ is said to have open lower sections if the set $T^{-1}(y):=\{x \in X \mid y \in T(x)\}$ is open in $X$ for every $y \in X$.

Let us recall the following useful fixed-point theorem for set-valued mappings.

Theorem 5.10 ([28], Browder). Let $C$ be a non-empty, convex and compact subset of the Hausdorff topological vector space $X$. If the set-valued mapping $T: C \rightrightarrows C$ has non-empty and convex values and has open lower sections, then $T$ attains a fixed-point, that is, there exists an element $x \in C$ such that

$$
x \in T(x) .
$$

Recall that the non-linear Tammer-Weidner function $\varphi^{K, e}: Y \rightarrow \mathbb{R}$ is defined by

$$
\varphi^{K, e}(y):=\inf \{t \in \mathbb{R} \mid y \in t e-K\}, \quad \text { for every } y \in Y,
$$

where $e \in \operatorname{int} K$ is a fixed element; compare Definition 2.14. While the proof of Theorem 5.7 made use of a linear scalarization, the alternative proof of Theorem 5.9, which has been proposed in [42], uses the equivalent formulation (3.10), which consists of finding an element $x \in C$ such that

$$
\varphi^{K, e}\left(\langle F x, y-x\rangle_{Y}\right) \geq 0, \quad \text { for every } \quad y \in C .
$$

Recall that, due to the representability condition of $\varphi^{K, e}$, the solution sets of (3.10) and (2.1) coincide. In [42, Theorem 7], the following alternative proof for Theorem 5.9 is given: 
Proof. Let us define the mapping $\eta: X \times X \rightarrow Y$ by $\eta(x, y):=\langle F x, y-x\rangle_{Y}$ for every $(x, y) \in X \times X$ and a family of set-valued mappings $T_{n}: C \rightrightarrows C$ by

$$
T_{n}(x):=\left\{y \in C \mid \varphi^{K, e} \circ \eta(x, y)-\min _{y^{\prime} \in C} \varphi^{K, e} \circ \eta\left(x, y^{\prime}\right)<\frac{1}{n}\right\}, \quad \text { for every } \quad x \in C .
$$

If we can ensure that every $T_{n}$ admits a fixed point, that is, we can find $x_{n} \in C$ such that

$$
x_{n} \in T_{n}\left(x_{n}\right),
$$

then the proof is complete. Indeed, since $C$ is compact, there is a subsequence, again denoted by $\left\{x_{n}\right\}$ such that $x_{n} \rightarrow x$ and $x \in C$. Therefore, passing in (5.13) to the limit yields

$$
\min _{y^{\prime} \in C} \varphi^{K, e} \circ \eta\left(x, y^{\prime}\right) \geq 0,
$$

or equivalently $\varphi^{K, e} \circ \eta\left(x, y^{\prime}\right) \geq 0$ for every $y^{\prime} \in C$. This follows from the fact that $F$ and $\varphi^{K, e}$ are continuous. Since $\varphi^{K, e}$ is strictly int $K$-monotone; see Theorem 2.4, we conclude

$$
\eta\left(x, y^{\prime}\right)=\left\langle F x, y^{\prime}-x\right\rangle_{Y} \notin-\operatorname{int} K, \quad \text { for every } \quad y^{\prime} \in C,
$$

that is, the limit point $x \in C$ is a solution of problem (2.1). It therefore remains to show that every $T_{n}$ has a fixed-point. Thus, we are going to show that the conditions of Browder's fixed-point Theorem 5.10 are satisfied.

(I). Let us show that every $T_{n}$ has non-empty and convex values. Let $x \in C$ be arbitrarily chosen. Evidently, the composition $\varphi^{K, e} \circ \eta(x, \cdot): X \rightarrow \mathbb{R}$ is continuous. Since $C$ is compact, $T_{n}(x)$ is non-empty. For the convexity of $T_{n}(x)$, let $y_{1}, y_{2} \in C$ and $0<t<1$. We define

$$
d_{j}:=\frac{1}{n}-\varphi^{K, e} \circ \eta\left(x, y_{j}\right)+\min _{y^{\prime} \in C} \varphi^{K, e} \circ \eta\left(x, y^{\prime}\right)>0, \quad \text { for } \quad j=1,2 .
$$

and $\lambda:=\varphi^{K, e} \circ \eta\left(x, y_{1}\right)+\frac{2}{n}$, where we assume without loss of generality that $d_{1}<d_{2}$. Thus,

$$
y_{j} \in \operatorname{lev}_{\varphi^{K, e} \circ \eta(x, \cdot)}(\lambda):=\left\{y \in C \mid \varphi^{K, e} \circ \eta(x, y) \leq \lambda\right\}
$$

for $j=1,2$. Since $\varphi^{K, e} \circ \eta(x, \cdot)$ is convex, the level set $\operatorname{lev}_{\varphi^{K, e} \circ \eta(x, \cdot)}(\lambda)$ is convex as well; see, for example, $[108,126]$. This implies

$$
\varphi^{K, e} \circ \eta\left(x, t y_{1}+(1-t) y_{2}\right)-\min _{y^{\prime} \in C} \varphi^{K, e} \circ \eta\left(x, y^{\prime}\right) \leq \frac{1}{n},
$$

which shows that $T_{n}(x)$ is convex.

(II). Let us show that every $T_{n}$ has open lower sections. Assume that there is some $m \in \mathbb{N}$ such that $T_{m}$ does not have open lower sections. In other words, we can find $x, y \in C$ with $x \in T_{m}^{-1}(y)$ and a sequence $\left\{x_{n}\right\} \subseteq C$ with $x_{n} \notin T_{m}^{-1}(y)$ such that $x_{n} \rightarrow x$. Since it holds

$$
T_{m}^{-1}(y)=\left\{x \in C \mid y \in T_{m}(x)\right\}=\left\{x \in C \mid \varphi \circ \eta(x, y)-\min _{y^{\prime} \in C} \varphi \circ \eta\left(x, y^{\prime}\right)<\frac{1}{m}\right\},
$$

we conclude that

$$
\varphi^{K, e} \circ \eta\left(x_{n}, y\right)-\min _{y^{\prime} \in C} \varphi^{K, e} \circ \eta\left(x_{n}, y^{\prime}\right) \geq \frac{1}{m} .
$$

Since $(x, y) \mapsto \varphi^{K, e} \circ \eta(x, y)$ is continuous and $C$ is compact, we conclude

$$
\lim _{n \rightarrow+\infty}\left[\varphi^{K, e} \circ \eta\left(x_{n}, y\right)-\min _{y^{\prime} \in C} \varphi^{K, e} \circ \eta\left(x_{n}, y^{\prime}\right)\right]=\varphi^{K, e} \circ \eta(x, y)-\min _{y^{\prime} \in C} \varphi^{K, e} \circ \eta\left(x, y^{\prime}\right) \geq \frac{1}{m},
$$

which contradicts the fact that $x \in T_{m}^{-1}(y)$. Hence, all set-valued mappings $T_{n}$ have open lower sections. (III). We have finally shown that every $T_{n}$ satisfies the assumptions of Theorem 5.10 such that (5.13) holds. The proof is complete. 
Similar to the previous results, one can replace the boundedness (or compactness) condition for $C$ by a strong $v$-coercivity condition of $F$.

Definition 5.6. Besides assumption (A), let $F: X \rightarrow \mathrm{L}(X, Y)$ be a given mapping. $F$ is said to be strongly $v$-coercive if there exists a non-empty and compact subset $B \subseteq X$ and an element $y_{0} \in B \cap C$ such that

$$
\left\langle F y_{0}, y_{0}-y\right\rangle_{Y} \in \operatorname{int} K, \quad \text { for every } \quad y \in C \backslash B .
$$

Theorem 5.11 ([189, Theorem 1]). Besides assumption (A), let $F: X \rightarrow \mathrm{L}(X, Y)$ be a continuous and strongly v-coercive mapping. Then, the vector variational inequality (2.1) has a solution.

Proof. We follow the proof of Theorem 5.9. In order to prove that (2.1) has a solution, it is enough to show that the intersection (5.12) is non-empty, where the set-valued mapping $G: C \rightrightarrows X$ is again defined as

$$
G(y):=\left\{x \in C \mid\langle F x, y-x\rangle_{Y} \notin-\operatorname{int} K\right\}, \quad \text { for every } \quad y \in C .
$$

We have already shown that $G$ is a KKM-mapping with non-empty and closed values; compare the proof of Theorem 5.9. It remains to show that there is an element $y_{0} \in C$ such that $G\left(y_{0}\right)$ is compact. Indeed, we have

$$
G\left(y_{0}\right) \subseteq B,
$$

where $y_{0} \in B \cap C$ and $B \subseteq X$ are given by the coercivity condition of this theorem. Thus, as a closed subset of the compact set $B, G\left(y_{0}\right)$ is compact in $X$. Consequently, the set-valued mapping $G: C \rightrightarrows X$ satisfies the assumptions of Lemma 5.1, which ensures that the intersection (5.12) is non-empty. The proof is complete.

Remark 5.9. Similar to the previous section, the strong $v$-coercivity of $F$ ensures the compactness of the set $G\left(y_{0}\right)$ only, while the boundedness of $C$ implies the weak compactness of all sets $G(y), y \in C$.

As a consequence of Browder's fixed-point Theorem 5.10, we have the following result by Fan.

Lemma 5.2 ([77], Fan section lemma). Let C be a non-empty subset of the Hausdorff topological vector space $X$. Let $A$ be a non-empty subset of $X \times X$. Assume further that the following conditions hold:

(i) For each $x \in C$, we have $(x, x) \in A$.

(ii) For each $y \in C$, the set $A(y):=\{x \in C \mid(x, y) \in A\}$ is closed in $C$.

(iii) For each $x \in C$, the set $B(x):=\{y \in C \mid(x, y) \notin A\}$ is convex or empty.

(iv) There exists a non-empty, convex and compact subset $B \subseteq X$ such that $\{x \in C \mid(x, y) \in A$ for every $y \in$ $B\}$ is compact in $C$.

Then, there exists an element $x \in C$ such that $\{x\} \times C \subseteq A$.

Remark 5.10. (i) Lemma 5.2 can be proven by using the Fan-KKM Lemma 5.1; compare Theorem 2.1 in [166].

(ii) Condition (iv) of Lemma 5.2 can be dropped if the set $C$ is assumed to be compact; see [64].

By using the Fan section lemma, we have the following alternative proof for Theorem 5.11, which can be found in [151, Theorem 2.2]:

Proof. Let us define the non-empty set

$$
A:=\left\{(x, y) \in C \times C \mid\langle F x, y-x\rangle_{Y} \notin-\operatorname{int} K\right\} \subseteq X \times X .
$$

Evidently, any element $x \in C$ satisfying

$$
\{x\} \times C \subseteq A
$$


is a solution of problem (2.1). In order to ensure the existence of such an element, we are going to show that all requirements of Lemma 5.2 are fulfilled, where

$$
A(y):=\{x \in C \mid(x, y) \in A\} \quad \text { and } \quad B(x):=\{y \in C \mid(x, y) \notin A\},
$$

for every $x, y \in C$. Condition (i) of this lemma follows from the properness of the cone $K$; see Remark 2.4. For (ii), let $y \in C$ and consider a sequence $\left\{x_{n}\right\}$ in $A(y)$ with $x_{n} \rightarrow x$. Since $F$ is continuous and the sets $C$ and $Y \backslash(-$ int $K)$ are closed, $\left\langle F x_{n}, y-x_{n}\right\rangle_{Y} \rightarrow\langle F x, y-x\rangle_{Y}$ and $x \in C$. We further have $\langle F x, y-x\rangle_{Y} \notin$ $-\operatorname{int} K$. Thus, $A(y)$ is closed. Condition (iii) follows from the fact that for fixed $x \in X$, the mapping $\langle F x,-x\rangle_{Y}: X \rightarrow Y$ is $K$-convex. For (iv), we consider the set $M:=\{x \in C \mid(x, y) \in A$ for every $y \in B\}$, where $B \subseteq X$ is a non-empty compact set, given by the $v$-coercivity condition. Let us show

$$
M \subseteq B .
$$

For this purpose, let $x \in C \backslash B$. Then by the $v$-coercivity, there exists an element $y_{0} \in B \cap C$ such that $\left\langle F x, y_{0}-x\right\rangle_{Y} \in \operatorname{int} K$, that is, $\left(x, y_{0}\right) \notin A$ and therefore $x \notin M$. Since $M=\bigcap_{y \in C} A(y)$ is the intersection of closed sets and therefore closed, $M$ is compact as a closed subset of $B$. By Lemma 5.2, there exists $x \in C$ such that (5.14) holds. The proof is complete.

Remark 5.11. Note that in $[7,8]$, the authors use Fan section Lemma 5.2 to prove the existence of solutions for vector variational inequalities with respect to a variable domination structure; compare also Section 11.2 of this paper.

The next existence result for the vector variational inequality (2.1) is based on Brouwer's famous fixed-point theorem, which we recall here.

Theorem 5.12 (Brouwer, [197, Proposition 2.6]). Every continuous mapping $T: B \rightarrow B$ from a nonempty, convex and compact subset of a finite-dimensional Euclidean space into itself has a fixed-point, that is, there exists an element $x \in B$ such that

$$
T(x)=x .
$$

Note that, in the following theorem, the mapping $F$ is neither assumed to be $K$-monotone nor continuous.

Theorem 5.13 ([130, Theorem 4.1]). Besides assumption (A), let $F: X \rightarrow \mathrm{L}(X, Y)$ be a given mapping. If $C$ is compact and for every $y \in C$, the set

$$
H(y):=\left\{x \in C \mid\langle F x, y-x\rangle_{Y} \in-\operatorname{int} K\right\}
$$

is open in $X$, then the vector variational inequality (2.1) has a solution.

Proof. Assume by contradiction that problem (2.1) has no solution, that is, for every $x \in C$, there exists an element $y \in C$ such that $\langle F x, y-x\rangle_{Y} \in-\operatorname{int} K$. Thus, $H(y)$ is a non-empty subset of $C$ for every $y \in C$. We therefore have $\bigcup_{y \in C} H(y) \subseteq C$ and the reverse inclusion $C \subseteq \bigcup_{y \in C} H(y)$ follows from the claim of contradiction. Consequently,

$$
C=\bigcup_{y \in C} H(y) .
$$

In other words, we have shown that $\{H(y) \mid y \in C\}$ is an open cover for $C$. Since $C$ is compact, there exists $k \in \mathbb{N}$ and a finite subcover $\left\{y_{1}, \ldots, y_{k}\right\} \subseteq C$ such that

$$
C=\bigcup_{j=1}^{k} H\left(y_{j}\right) \text {. }
$$


Let $\left\{g_{1}, \ldots, g_{k}\right\}$ be a partition of unity [197] subordinate to this cover. Recall that for $j=1, \ldots, k$ the function $g_{j}: C \rightarrow[0,1]$ is continuous and enjoys the properties

$$
\begin{aligned}
g_{j}(x) & =0, \quad \text { for every } & x \in X \backslash H\left(y_{j}\right), \\
\sum_{j=1}^{k} g_{j}(x) & =1, \quad \text { for every } & x \in X .
\end{aligned}
$$

Let $B:=\operatorname{conv}\left\{y_{1}, \ldots, y_{k}\right\}$ and define a mapping $g: B \rightarrow X$ by

$$
g(x):=\sum_{j=1}^{k} g_{j}(x) y_{j}, \quad \text { for every } \quad x \in B .
$$

It is easily seen that $g$ maps $B$ into $B$. Since $g: B \rightarrow B$ is continuous and $B \subseteq \operatorname{lin}\left\{y_{1}, \ldots, y_{k}\right\}$ is a nonempty, convex and compact subset of a finite-dimensional space, Brouwer's fixed-point theorem implies the existence of a fixed point $x \in B$, that is,

$$
g(x)=x .
$$

Let us show that (5.15) is impossible. Indeed, using the fact that $\sum_{j=1}^{k} g_{j}(x) x=x$, we deduce from (5.15) that

$$
0=\langle F x, g(x)-x\rangle_{Y}=\left\langle F x, \sum_{j=1}^{k} g_{j}(x) y_{j}-x\right\rangle_{Y}=\sum_{j=1}^{k} g_{j}(x)\left\langle F x, y_{j}-x\right\rangle_{Y} .
$$

Since we have in particular $x \in H\left(y_{j}\right)$ for $j=1, \ldots, k$, the right-hand side of the above equation lies in $-\operatorname{int} K$. We have therefore shown that $0 \in \operatorname{int} K$, which is impossible since the cone $K$ is assumed to be proper; see Remark 2.4. This shows that the vector variational inequality (2.1) has a solution. The proof is complete.

Remark 5.12. Some other results using similar arguments can be found in [81, Theorem 2.1] and [176, Theorem 4.1].

5.3. Regularization. The previous sections have extensively shown that coercivity conditions of the data of problem (2.1) are crucial to ensure the existence of solutions of it. However, the solvability of problem (2.1) can be still studied, even though the data of the problem do not satisfy any coercivity condition. For this purpose, the authors in [115] have proposed an extension of the well-known BrowderTikhonov regularization method [144], which has been used extensively for scalar (quasi) variational inequalities; see, for example, $[2,18,144]$ and the references therein. To be precise, assume again that vector variational inequality (2.1) is non-coercive and let a mapping $R: X \rightarrow \mathrm{L}(X, Y)$ and a sequence $\left\{\varepsilon_{n}\right\} \subseteq \mathbb{R}_{>}$of parameters be given. Instead of problem (2.1), we consider the family of regularized vector variational inequalities of finding an element $x_{n} \in C$ such that

$$
\left\langle F x_{n}+\varepsilon_{n} R x_{n}, y-x_{n}\right\rangle_{Y} \notin-\operatorname{int} K, \quad \text { for every } y \in C .
$$

Any solution of (5.16) is said to be a regularized solution. In the above, $R$ is the regularizing mapping and $\varepsilon_{n}$ is the regularization parameter to problem (5.16). Note that this family of problems evolves from the vector variational inequality (2.1) if one replaces $F$ by the perturbed mapping

$$
F+\varepsilon_{n} R: X \rightarrow \mathrm{L}(X, Y) .
$$

Due to the nice features of $R$, which will be specified shortly, the mapping $F+\varepsilon_{n} R$ has significantly better properties than $F$ and every regularized problem (5.16) has a solution $x_{n}$. This allows us to study the sequence $\left\{x_{n}\right\}$ of regularized solutions, which, under some boundedness conditions, has a weakly or strongly convergent subsequence and any limit point is a solution of the vector variational inequality (2.1). By this, we can ensure the existence of solutions of non-coercive vector variational problems. 
The following example is based on the one in [115]. It presents a finite-dimensional vector variational inequality of the type (2.2), which has a solution although the data of the problem do not satisfy any coercivity condition of the previous section.

Example 5.3 ([115, Example 2]). Consider the finite-dimensional vector variational inequality (2.2) with $l=k=2$ and $C=\mathbb{R}_{\geq}^{2}$. Assume that the mapping $F: \mathbb{R}^{2} \rightarrow \operatorname{Mat}_{2 \times 2}(\mathbb{R})$ is given by

$$
F x:=\left(\begin{array}{cc}
f\left(x_{1}\right) & 0 \\
0 & g\left(x_{2}\right)
\end{array}\right), \quad \text { for every } \quad x \in \mathbb{R}^{2},
$$

where the monotone and continuous mappings $f, g: \mathbb{R} \rightarrow \mathbb{R}$ enjoy the following properties:

$$
f(x)=0 \text { for } x \geq 0, \quad g(0)=0 \quad \text { and } \quad \lim _{x \rightarrow \pm \infty} g(x)<+\infty .
$$

By this special choice of the data, problem (2.2) simplifies to: find an element $x \in \mathbb{R}_{\geq}^{2}$ such that

$$
\left(\begin{array}{l}
f\left(x_{1}\right)\left(y_{1}-x_{1}\right) \\
g\left(x_{2}\right)\left(y_{2}-x_{2}\right)
\end{array}\right) \notin-\operatorname{int} \mathbb{R}_{\geq}^{2}, \quad \text { for every } \quad y \in \mathbb{R}_{\geq}^{2} .
$$

It is easily seen that the mapping $F: \mathbb{R}^{2} \rightarrow \operatorname{Mat}_{2 \times 2}(\mathbb{R})$ is $\mathbb{R}_{\geq}^{2}$-monotone and $v$-hemicontinuous. However, one can show, compare [115, Example 2], that none of the coercivity conditions of the previous sections are satisfied. We are therefore unable to apply any of these existence results. But nevertheless, it is easy to check that the zero vector $(0,0)^{\top}$ solves problem (5.17).

The next theorem ensures, that every regularized problem (5.16) has a solution, even if one does not assume that the mapping $F$ enjoys any coercivity condition.

Theorem 5.14 ([115, Theorem 7]). Besides assumption (A), let $F, R: X \rightarrow \mathrm{L}(X, Y)$ be given mappings.

(i) Assume that $F$ and $R$ are $K$-monotone and $v$-hemicontinuous. If either $R$ is weakly coercive or $C$ is bounded, then the regularized vector variational inequality (5.16) has a solution.

(ii) Assume that $F$ and $R$ are strongly continuous. If $R$ is $\kappa$-coercive with respect to the functional $s \in K^{*} \backslash\{0\}$ and it holds that

$$
\langle s \circ F x, x\rangle_{X^{*}, X} \geq 0, \quad \text { for every } \quad x \in C,
$$

then the regularized vector variational inequality (5.16) has a solution.

Proof. (i). It is easily seen that the mapping $F+\varepsilon_{n} R$ is $K$-monotone and $v$-hemicontinuous as a sum of two. If $C$ is bounded in addition, then (5.16) has a solution; see Theorem 5.1. Now, letting $C$ be unbounded but $R$ weakly coercive, there are $x_{0} \in C$ and $s \in$ qi $K^{*}$ such that (5.10) holds. Finally, using the fact that $s \circ F: X \rightarrow X^{*}$ is monotone, it follows for every $x \in C$

$$
\begin{aligned}
\left\langle s \circ F x-s \circ F x_{0}, x-x_{0}\right\rangle_{X^{*}, X}+\varepsilon_{n}\left\langle s \circ R x-s \circ R x_{0}, x-x_{0}\right\rangle_{X^{*}, X} \\
\geq \varepsilon_{n}\left\langle s \circ R x-s \circ R x_{0}, x-x_{0}\right\rangle_{X^{*}, X},
\end{aligned}
$$

implying that $F+\varepsilon_{n} R$ is weakly coercive. Consequently, Theorem 5.7 ensures the existence of solutions of problem (5.16).

(ii). The key idea is to apply Theorem 5.8. Since $F+\varepsilon_{n} R$ is strongly continuous, it remains to show that $F+\varepsilon_{n} R$ is $\kappa$-coercive with respect to $s \in K^{*} \backslash\{0\}$. Indeed, it holds

$$
\langle s \circ F x, x\rangle_{X^{*}, X}+\varepsilon_{n}\langle s \circ R x, x\rangle_{X^{*}, X} \geq \varepsilon_{n}\langle s \circ R x, x\rangle_{X^{*}, X} \geq \varepsilon_{n}\|s\|_{Y^{*}} \kappa(x),
$$

for every $x \in C$, implying that (5.16) has a solution.

The next result shows that the strong limit point of any sequence of regularized solutions is a solution of the vector variational inequality (2.1). 
Theorem 5.15 ([115, Theorem 8]). Besides assumption (A), let $F, R: X \rightarrow \mathrm{L}(X, Y)$ be given mappings. Then, the following statements hold:

(i) Assume that $F$ and $R$ are $K$-monotone and $v$-hemicontinuous and $R$ is weakly coercive. If there is a strongly convergent sequence of regularized solutions, then the vector variational inequality (2.1) has a solution.

(ii) Assume that $F$ and $R$ are strongly continuous, $R$ is $\kappa$-coercive with respect to the functional $s \in K^{*} \backslash\{0\}$ and it holds that

$$
\langle s \circ F x, x\rangle_{X^{*}, X} \geq 0, \quad \text { for every } \quad x \in C .
$$

If there is a strongly convergent sequence of regularized solutions, then the vector variational inequality (2.1) has a solution.

Proof. Let us denote the sequence of regularized solutions by $\left\{x_{n}\right\}$. Note that in both cases (i) and (ii), the sequence is well-defined in the sense that every problem (5.16) has a solution; compare Theorem 5.14 .

(i). By definition, we have $x_{n} \in C$ and

$$
\left\langle F x_{n}+\varepsilon_{n} R x_{n}, y-x_{n}\right\rangle_{Y} \notin-\operatorname{int} K, \quad \text { for every } \quad y \in C .
$$

From the $K$-monotonicity and $v$-hemicontinuity of $F+\varepsilon_{n} R$ follows that $x_{n}$ satisfies equivalently

$$
\left\langle F y+\varepsilon_{n} R y, y-x_{n}\right\rangle_{Y} \notin-\operatorname{int} K, \quad \text { for every } y \in C,
$$

see Lemma 4.1. Let us denote the strong limit point of $\left\{x_{n}\right\}$ by $x$. Since $\varepsilon_{n} \downarrow 0$ and $x_{n} \rightarrow x$, it is easy to check that for fixed $y \in C$ it holds that

$$
\left\langle F y+\varepsilon_{n} R y, y-x_{n}\right\rangle_{Y} \rightarrow\langle F y, y-x\rangle_{Y} \text { in } Y,
$$

compare Proposition 2.4 (vi). Since $\left\langle F y+\varepsilon_{n} R y, y-x_{n}\right\rangle_{Y} \in Y \backslash(-\operatorname{int} K)$ and the set $Y \backslash(-\operatorname{int} K)$ is closed, we conclude $\langle F y, y-x\rangle_{Y} \notin-\operatorname{int} K$. Finally, applying Lemma 4.1 once again, we conclude that $x \in C$ is a solution of the vector variational inequality (2.1).

(ii). Denote the strong limit point of $\left\{x_{n}\right\}$ by $x$, that is, $x_{n} \rightarrow x$. Due to the strong continuity of $F+\varepsilon_{n} R$, it holds that $F x_{n}+\varepsilon_{n} R x_{n} \rightarrow F x$ in $\mathrm{L}(X, Y)$. Therefore, we can pass in (5.18) to the limit, that is, we have

$$
\left\langle F x_{n}+\varepsilon_{n} R x_{n}, y-x_{n}\right\rangle_{Y} \rightarrow\langle F x, y-x\rangle_{Y} \text { in } Y .
$$

Thus, $x \in C$ solves the vector variational inequality (2.1). The proof is complete.

Remark 5.13. (i) The above proof shows that the strong limit point of any convergent sequence of regularized solutions solves the vector variational inequality (2.1).

(ii) A similar technique for finite-dimensional vector variational inequalities has also been proposed in [167]. In this paper, Luong proposes to consider a family of so-called penalized finite-dimensional vector variational inequalities. Note that the results in [167] still require the coercivity of $F$, which make a penalization approach superfluous. Luong shows that every penalized problem has a solution and that the sequence of penalized solutions converges to a solution of the original problem (2.2), where the set $C$ is given by

$$
C:=\left\{x \in \mathbb{R}^{l} \mid g_{j}(x) \leq 0 \text { for } j=1, \ldots, k\right\},
$$

with continuous functions $g_{j}: \mathbb{R}^{l} \rightarrow \mathbb{R}$ for $j=1, \ldots, k$. Due to this special choice of the data, Luong defined a penalization mapping $R: \mathbb{R}^{l} \rightarrow \operatorname{Mat}_{k \times l}(\mathbb{R})$ by $R:=(\nabla P, \ldots, \nabla P)^{\top}$, where $\nabla P \in \mathbb{R}^{l}$ denotes the Fréchet-derivative of $P: \mathbb{R}^{l} \rightarrow \mathbb{R}$, given by

$$
P(x):=\sum_{j=1}^{k}\left[\max \left\{0, g_{j}(x)\right\}\right]^{2}, \quad \text { for every } \quad x \in \mathbb{R}^{l} .
$$


Therefore, the proofs in [167] extensively use the fact that the convex and Fréchet differentiable mapping $P$ enjoys the property of a penalty mapping for $C$, namely

$$
P(x)=0 \text { for } x \in C \quad \text { and } \quad P(x)>0 \text { else. }
$$

Example 5.4. We consider again the setting of Example 5.3. Let us show that the vector variational inequality (5.17) has a solution. For this purpose, we introduce a regularizing mapping $R: \mathbb{R}^{2} \rightarrow \mathrm{Mat}_{2 \times 2}(\mathbb{R})$ by

$$
R x:=\left(\begin{array}{cc}
x_{1} & 0 \\
0 & x_{2}
\end{array}\right), \quad \text { for every } \quad x \in \mathbb{R}^{2}
$$

Further, let $\left\{\varepsilon_{n}\right\} \subseteq \mathbb{R}_{>}$be a sequence such that $\varepsilon_{n} \downarrow 0$. It is easily seen that the regularizer $R$ is $\mathbb{R}_{\geq}^{2}$ monotone and $v$-hemicontinuous. If we let $s:=(1,1)^{\top}$ and $x_{0}:=(0,0)^{\top}$, then it holds that

$$
\frac{\left\langle s^{\top} R x-s^{\top} R x_{0}, x-x_{0}\right\rangle}{\left\|x-x_{0}\right\|_{2}}=\|x\|_{2}, \quad \text { for every } \quad x \in \mathbb{R}^{2} .
$$

Thus, $R$ is weakly coercive. Taking into account Theorem 5.14, the following family of regularized vector variational inequalities has a solution: find an element $x_{n} \in \mathbb{R}_{\geq}^{2}$ such that

$$
\left(\begin{array}{c}
\left(f\left(x_{1}^{n}\right)+\varepsilon_{n} x_{1}^{n}\right)\left(y_{1}-x_{1}^{n}\right) \\
\left(g\left(x_{2}^{n}\right)+\varepsilon_{n} x_{2}^{n}\right)\left(y_{2}-x_{2}^{n}\right)
\end{array}\right) \notin-\operatorname{int} \mathbb{R}_{\geq}^{2}, \quad \text { for every } \quad y \in \mathbb{R}_{\geq}^{2} .
$$

Indeed, a solution is given by $x_{n}=\left(0, \varepsilon_{n}^{2}\right)^{\top}$ and it holds that

$$
\left(0, \varepsilon_{n}^{2}\right)^{\top} \rightarrow(0,0)^{\top}
$$

Therefore, in view of Theorem 5.15, the limit point $(0,0)^{\top}$ is a solution of problem (5.17).

\section{ApPLICATIONS IN MULTI-OBJECTIVE OPTIMIZATION}

In this section, we are going to show that vector variational inequalities can be used to study multiobjective optimization problems. Such relations are very useful since one can transfer techniques and ideas from one field to the other; see, for example, [73, 105]. Within the last years, several authors have tried to show that vector variational inequalities can be used to solve vector equilibrium problems. However, one has to be very careful since there are some incorrect results in this research area; compare the Sections 3 and 4 in the survey paper [71].

For further use, we need the following two definitions.

Definition 6.1 ([126, Definition 2.4]). Let $X$ and $Y$ be real linear spaces and let $K$ be a convex cone in $Y$. A mapping $\psi: X \rightarrow Y$ is said to be $K$-convex if for all $x, y \in X$ and every $\lambda \in[0,1]$

$$
\lambda \psi(x)+(1-\lambda) \psi(y)-\psi(\lambda x+(1-\lambda) y) \in K .
$$

Remark 6.1. (i) Using the ordering relation $\leq_{K}$, see Definition 2.10, the above equation becomes $\lambda \psi(x)+(1-\lambda) \psi(y) \leq_{K} \psi(\lambda x+(1-\lambda) y)$ for all $x, y \in X$ and $\lambda \in[0,1]$.

(ii) A mapping $\psi: \mathbb{R}^{l} \rightarrow \mathbb{R}^{k}$ is $\mathbb{R}_{\geq}^{k}$-convex if and only if all components are convex functions from $\mathbb{R}^{l}$ to $\mathbb{R}$.

Definition 6.2 ([126, Definition 2.12]). Let $X$ and $Y$ be real normed spaces and let $\psi: X \rightarrow Y$ be a given mapping. The directional derivative of $\psi$ at $x \in X$ in the direction $h \in X$ is given by

$$
\delta \psi(x ; h):=\lim _{t \rightarrow 0} \frac{\psi(x+t h)-\psi(x)}{t},
$$


provided this limit exists. If $\delta \psi(x ; h)$ exists for every $h \in X$, and if the mapping $D_{\mathrm{G}} \psi(x): X \rightarrow Y$ defined by

$$
\left\langle D_{\mathrm{G}} \psi(x), h\right\rangle_{Y}:=D_{\mathrm{G}} \psi(x) h:=\delta \psi(x ; h)
$$

is linear and continuous, then we say that $\psi$ is Gateaux-differentiable at $x$, and we call $D_{\mathrm{G}} \psi(x)$ the Gâteaux-derivative of $\psi$ at $x$. If $\psi$ is Gâteaux-differentiable at every point $x \in X$, then we say that $\psi$ is Gâteaux-differentiable. Similar, we define the right-and left-handed Gâteaux-derivative of $\psi$ at $x$, which will be denoted by $D_{\mathrm{G}}^{+} \psi(x)$ and $D_{\mathrm{G}}^{-} \psi(x)$, respectively.

The following result shows that solving a smooth multi-objective optimization problem is equivalent to solving a vector variational inequality.

Theorem 6.1 ([41, Proposition 3.3]). Besides assumption (A), let $\psi: X \rightarrow Y$ be a given mapping.

(i) If $\psi$ is right-handed Gâteaux-differentiable at $x \in C$ with derivative $D_{\mathrm{G}}^{+} \psi(x)$ and

$$
x \in \operatorname{WEff}(\psi[C], K)
$$

is a weakly efficient element, then $x \in C$ satisfies the vector variational inequality

$$
\left\langle D_{\mathrm{G}}^{+} \psi(x), y-x\right\rangle_{Y} \notin-\operatorname{int} K, \quad \text { for every } y \in C .
$$

(ii) Conversely, if $x \in C$ solves the vector variational inequality (6.2) and $\psi$ is $K$-convex in addition, then $x$ satisfies (6.1).

Proof. (i). Let $x \in \operatorname{WEff}(\psi[C], K)$. Since $C$ is convex, we deduce

$$
\frac{1}{t}[\psi(x+t(y-x))-\psi(x)] \notin-\operatorname{int} K,
$$

for every $y \in C$ and $t \in(0,1)$. Passing to the limit $t \downarrow 0$ yields $\left\langle D_{\mathrm{G}}^{+} \psi(x), y-x\right\rangle_{Y} \notin-\operatorname{int} K$ for every $y \in C$. (ii). Conversely, let $x \in C$ solve the vector variational inequality (6.2). Due to the $K$-convexity of $\psi$, it holds in particular

$$
\psi(y)-\psi(x)-\frac{1}{t}[\psi(x+t(y-x))-\psi(x)] \in \operatorname{int} K,
$$

for every $y \in C$. Passing to the limit $t \downarrow 0$ and combining the resulting inequality with (6.2) implies $\psi(x)-\psi(y) \notin \operatorname{int} K$. The proof is complete.

Remark 6.2. (i) Theorem 6.1 is known as Fermat's rule for multi-objective optimization problems; see Section 2 in [195].

(ii) The previous theorem stats that a multi-objective optimization problem with a smooth, that is, Gâteaux-differentiable and $K$-convex objective mapping, is equivalent to a vector variational inequality of the type (2.1).

(iii) Investigations of relations between vector variational inequalities and (set-valued) multi-objective problems can also be found in $[12,13,61,92,107,150,153,154,172,182,192,190]$.

The following example is based on Example 3.7 in [115].

Example 6.1. Let $a^{1}, \ldots, a^{k}$ be different elements in $\mathbb{R}^{k}$. In this example, we are going to calculate the set of weak efficient points

$$
\operatorname{WEff}\left(\psi\left[\mathbb{R}^{l}\right], \mathbb{R}_{\geq}^{k}\right),
$$

where the objective mapping $\psi: \mathbb{R}^{l} \rightarrow \mathbb{R}^{k}$ is given by

$$
\psi(x):=\left(\begin{array}{c}
\frac{1}{2}\left\|x-a^{1}\right\|_{2}^{2} \\
\vdots \\
\frac{1}{2}\left\|x-a^{k}\right\|_{2}^{2}
\end{array}\right), \quad \text { for every } \quad x \in \mathbb{R}^{l} .
$$


As always, $\|\cdot\|_{2}$ denotes the Euclidean norm in $\mathbb{R}^{l}$. In order to calculate (6.3), we will use Theorem 6.1 and study an equivalent vector variational inequality. Let us therefore show that the objective mapping $\psi$ is $\mathbb{R}_{>}^{k}$-convex and Gâteaux-differentiable. Indeed, since all components of $\psi$ are convex functions from $\mathbb{R}^{l}$ to $\mathbb{R}$, the $\mathbb{R}_{\geq}^{k}$-convexity of $\psi$ follows; see Remark 6.1. Note that it holds

$$
\psi_{j}(x+t h)-\psi_{j}(x)=\left\|x+t h-a^{j}\right\|_{2}^{2}-\left\|x-a^{j}\right\|_{2}^{2}=t\left\langle x-a^{j}, h\right\rangle+\frac{1}{2} t^{2}\|h\|_{2}^{2}
$$

for every $x, y \in \mathbb{R}^{l}, t \in \mathbb{R}, j \in\{1, \ldots, k\}$. Thus,

$$
\delta \psi(x ; h)=\lim _{t \rightarrow 0} \frac{\psi(x+t h)-\psi(x)}{t}=\left(\begin{array}{c}
\left\langle x-a^{1}, h\right\rangle \\
\vdots \\
\left\langle x-a^{k}, h\right\rangle
\end{array}\right) \quad \text { and } \quad D_{\mathrm{G}} \psi(x)=\left(\begin{array}{c}
x-a^{1} \\
\vdots \\
x-a^{k}
\end{array}\right)
$$

Consequently, Theorem 6.1 states that problem (6.3) is equivalent to the following finite-dimensional vector variational inequality: find an element $x \in \mathbb{R}_{\geq}^{l}$ such that

$$
\left\langle D_{\mathrm{G}} \psi(x), y-x\right\rangle_{\mathbb{R}^{k}}=\left(\begin{array}{c}
\left\langle x-a^{1}, y-x\right\rangle \\
\vdots \\
\left\langle x-a^{k}, y-x\right\rangle
\end{array}\right) \notin-\operatorname{int} \mathbb{R}_{\geq}^{k}, \quad \text { for every } \quad y \in \mathbb{R}^{l} .
$$

But this is problem (2.3). Consequently,

$$
\operatorname{WEff}\left(\psi\left[\mathbb{R}^{l}\right], \mathbb{R}_{\geq}^{k}\right)=\mathrm{S}=\operatorname{conv}\left\{a^{1}, \ldots, a^{k}\right\} .
$$

Remark 6.3. The multi-objective optimization problem (6.3) is known in the literature as location problem and has various real-life applications; see [108, Chapter 4]. For instance, if $l=2$, then we can interpret problem (6.3), for example, in the following way: $a^{1}, \ldots, a^{k}$ are the locations of hospitals in a certain area. In order to build a new main office, we are looking for an appropriate location such that the air-line distance between the unknown location and all hospitals $a^{1}, \ldots, a^{k}$ is minimal simultaneously.

\section{INVERSE VECTOR VARIATIONAL INEQUALITIES}

This section is devoted to the study of inverse vector variational inequalities for vector variational inequality (2.1). The fundamental idea goes back to the work of Mosco [169], who introduced in 1972 a dual variational inequality for the variational inequality (1.3), using the Fenchel conjugate for convex functions. For this purpose, Mosco used the term dual in order to point out similarities to the duality principle in optimization; see $[25,95,106,108]$ and the references therein. However, it should be mentioned that the concept of duality has not been defined for a variational inequality yet, which is why one should call them inverse variational problems instead; see [73, 102]. In the last years, Mosco's idea has been adapted by several authors $[41,46,73,138,189,191]$ in order to derive inverse results for vector variational inequalities of the type (2.1) and related problems. Let us recall some of the main reasons to study inverse vector variational inequalities:

1. It has a tremendous aesthetic appeal.

2. It deepens the theoretical understanding of vector variational inequalities.

3. It provides the insight for devising effective computational methods and algorithms.

In what follows, we are going to introduce two inverse vector variational inequalities for problem (2.1). For this purpose, suppose that assumption (A) holds and denote by $\chi_{C}: X \rightarrow Y \cup\left\{+\infty_{Y}\right\}$ the generalized indicator mapping of the set $C$. Recall that $\chi_{C}(x):=0$ for $x \in C$ and $\chi_{C}(x):=+\infty_{Y}$ else. Evidently, we may rewrite problem (2.1) in the following way: find an element $x \in X$ such that

$$
\langle F x, y-x\rangle_{Y} \mathbb{L}_{\operatorname{int} K} \chi_{C}(x)-\chi_{C}(y), \quad \text { for every } y \in X .
$$


See $[25,73]$ for an introduction and useful conventions of the extended space $Y \cup\left\{ \pm \infty_{Y}\right\}$. Let in addition $F: X \rightarrow \mathrm{L}(X, Y)$ be injective. Then, the so-called adjoint mapping of $F$ is the mapping $F^{-}: \mathrm{L}(X, Y) \rightarrow X$, defined by

$$
F^{-} U:=F^{-1}(-U), \quad \text { for every } \quad U \in D\left(F^{-}\right) .
$$

Here, with some abuse of the notation, $F^{-1}$ denotes the inverse mapping of the bijection $F: X \rightarrow R(F)$. Note that the above definition of the adjoint mapping differs slightly from that in [189, Section 4].

In what follows, the next two definitions are crucial.

Definition 7.1 ([73, Definition 3.7]). Besides assumption (A), let $\psi: X \rightarrow Y \cup\left\{+\infty_{Y}\right\}$ be a $K$-convex mapping. An operator $U \in \mathrm{L}(X, Y)$ is called a weak subgradient of $\psi$ at $x \in X$ if $\psi(x) \neq+\infty_{Y}$ and

$$
\psi(y)-\psi(x)-\langle U, y-x\rangle_{Y} \not_{\operatorname{int} K} 0, \quad \text { for every } y \in X .
$$

The set of weak subgradients of $\psi$ at $x \in X$ will be denoted by $\partial \psi(x)$. If $\partial \psi(x)$ is non-empty, then $\psi$ is said to be weakly subdifferentiable at $x$. If $\psi$ is weakly subdifferentiable at every point of its effective domain, then $\psi$ is said to be weakly subdifferentiable.

Definition 7.2 ([73, Definition 3.9]). Besides assumption (A), let $\psi: X \rightarrow Y \cup\{+\infty Y\}$ be a $K$-convex mapping. The set-valued mapping $\psi^{*}: \mathrm{L}(X, Y) \rightrightarrows Y$, defined by

$$
\psi^{*}(U):=\operatorname{WMax}\left(\left\{\langle U, x\rangle_{Y}-\psi(x) \mid x \in \operatorname{dom}(\psi)\right\}, K\right) \quad \text { for every } U \in \mathrm{L}(X, Y),
$$

is called the weak conjugate of $\psi$, where $\operatorname{dom}(\psi):=\left\{x \in X \mid \psi(x) \neq+\infty_{Y}\right\}$ denotes the effective domain of $\psi$.

Remark 7.1. (i) It is easily seen that the generalized indicator mapping $\chi_{C}: X \rightarrow Y \cup\left\{+_{Y}\right\}$ is $K$-convex and weakly subdifferentiable on $C$.

(ii) The above definition recovers the well-known notion of Fenchel conjugate for functions $\psi: X \rightarrow$ $\mathbb{R} \cup\{+\infty\}$, compare [25, Definition 2.3.1], if we define $Y:=\mathbb{R}$ and $K:=\mathbb{R}_{\geq}$. Recall that the Fenchel conjugate of $\psi$ is the function $\psi^{*}: X^{*} \rightarrow \mathbb{R} \cup\{+\infty\}$, defined by

$$
\psi^{*}\left(x^{*}\right):=\sup _{x \in X}\left\{\left\langle x^{*}, x\right\rangle-\psi(x)\right\}=\sup _{x \in \operatorname{dom}(\psi)}\left\{\left\langle x^{*}, x\right\rangle-\psi(x)\right\}, \quad \text { for every } \quad x^{*} \in X^{*},
$$

where $\operatorname{dom}(\psi):=\{x \in X \mid \psi(x) \neq+\infty\}$ denotes the effective domain of $\psi$.

We are now in position to state the two inverse vector variational inequalities, which have been proposed in [73]. The first inverse vector variational inequality of (2.1) consists of finding an operator $U \in D\left(F^{-}\right)$such that

$$
\left\langle V-U,-F^{-} U\right\rangle_{Y} \aleph_{\text {int } K}^{1} \chi_{C}^{*}(U)-\chi_{C}^{*}(V), \quad \text { for every } \quad V \in \mathrm{L}(X, Y) \text { with } \chi_{C}^{*}(V) \neq \emptyset .
$$

In a similar way, the second inverse vector variational inequality consists of finding an operator $U \in$ $D\left(F^{-}\right)$such that

$$
\left\langle V-U,-F^{-} U\right\rangle_{Y} \npreceq_{\operatorname{int} K}^{2} \chi_{C}^{*}(U)-\chi_{C}^{*}(V), \quad \text { for every } \quad V \in \mathrm{L}(X, Y) .
$$

Here, the right-hand side of problem (7.1) and (7.2) is the Minkowski difference of the weak conjugates of the generalized indicator mapping $\chi_{C}$. Recall that the set relations $\aleph_{\text {int } K}^{1}$ and $\aleph_{\text {int } K}^{2}$ are defined for non-empty subset $A$ and $B$ of $Y$ in the following way: $A \AA_{\text {int } K}^{1} B$ if and only if for all $a \in A$, there is $b \in B$ such that $a \not_{\operatorname{int} K} b ; A \npreceq_{\text {int } K}^{2} B$ if and only if there is $a \in A$, such that for all $b \in B$ it holds $a \not Z_{\text {int } K} b$; compare Section 2. We further use the convention $A \aleph_{\text {int } K}^{2} \emptyset$ for every non-empty subset $A$ of $Y$. To the best of our knowledge, the first attempt to extend Mosco's idea to the vector case has been proposed in [189]. However, the main result in the paper of Yang, see Theorem 3 in [189], contains some certain errors. Consequently, the results in $[41,46,138,191]$, which copied the errors, are incorrect as well. Nevertheless, the ideas of [189] have been adapted in [73], where Elster et al. introduced the inverse 
vector variational inequalities (7.1) and (7.2). The basic idea is to embed the vector variational inequality (2.1) into the two inverse problems in the sense, that, under suitable assumptions, every solution of problem (7.1) generates one of (2.1), and every solution of problem (2.1) generates a solution of problem (7.2).

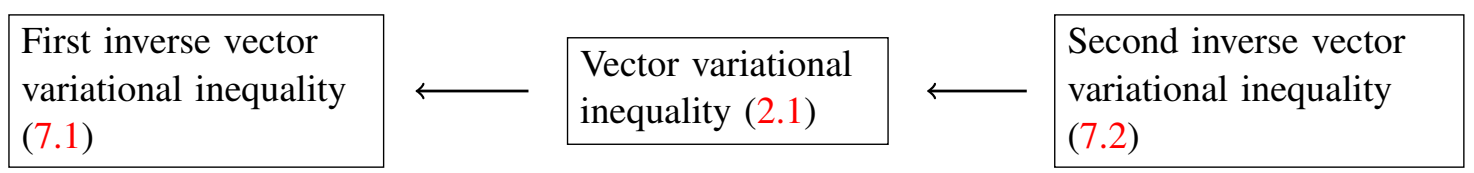

Definition 7.3 ([73, Definition 3.14]). Besides assumption (A), let $A$ and $B$ be non-empty subsets of $Y \cup\left\{ \pm_{Y}\right\}$. We say that $A$ and $B$ satisfy the weak $(A, B)$-domination property with respect to the cone $K$ if for every $b \in B \backslash\left\{+\infty_{Y}\right\}$ there exists $y^{0} \in \operatorname{WMax}(A, K)$ such that $y^{0} \geq_{K} b$.

The following result is a special case of [73, Theorem 4.3].

Theorem 7.1. Besides assumption (A), let $F: X \rightarrow \mathrm{L}(X, Y)$ be an injective mapping. Then, the following statements hold:

(i) If $x \in C$ is a solution of the vector variational inequality (2.1), then the operator $-F x \in D\left(F^{-}\right)$ solves the first inverse vector variational inequality (7.1).

(ii) Conversely, suppose that the operator $U \in D\left(F^{-}\right)$is a solution of the second inverse vector variational inequality (7.2) and define $x=F^{-} U$. If $x \in C$ and the sets

$$
A(x):=\left\{\langle-F x, y\rangle_{Y}-\chi_{C}(y) \mid y \in X\right\} \text { and } B(x):=\left\{-\langle F x, x\rangle_{Y}-\chi_{C}(x)\right\}
$$

satisfy the weak $(A(x), B(x))$-domination property, then $x$ is a solution of the vector variational inequality (2.1).

Proof. (i). Let $x \in C$ be a solution of problem (2.1), that is, we have

$$
-\langle F x, x\rangle_{Y}-\chi_{C}(x) \in \chi_{C}^{*}(-F x) .
$$

Assuming to the contrary that $-F x$ is not a solution of problem (7.1), we can find an operator $V_{0} \in$ $\mathrm{L}(X, Y)$ such that

$$
\left\langle V_{0}+F x,-x\right\rangle \preccurlyeq_{\operatorname{int} K}^{1} \chi_{C}^{*}(-F x)-\chi_{C}^{*}\left(V_{0}\right)
$$

and $\chi_{C}^{*}\left(V_{0}\right) \neq \emptyset$. Evidently, we have

$$
\chi_{C}(x)-\left\langle V_{0}, x\right\rangle_{Y} \preccurlyeq_{\text {int } K}^{1}-v_{0}, \quad \text { for every } \quad v_{0} \in \chi_{C}^{*}\left(V_{0}\right) .
$$

However, the above inequality implies $\chi_{C}\left(x_{0}\right)-\left\langle V_{0}, x_{0}\right\rangle_{Y}-\chi_{C}(x)+\left\langle V_{0}, x\right\rangle_{Y} \in$ int $K$ for some $x_{0} \in C$ which contradicts the fact that $\left\langle V_{0}, x_{0}\right\rangle_{Y}-\chi_{C}\left(x_{0}\right) \in \chi_{C}^{*}\left(V_{0}\right)$, showing that the operator $-F x$ solves problem (7.1). (ii). Let $U \in D\left(F^{-}\right)$be a solution of problem (7.2). Since $x=F^{-} U$, we have $U=-F x$ and it holds

$$
-\langle V+F x, x\rangle_{Y} \aleph_{\operatorname{int} K}^{2} \chi_{C}^{*}(-F x)-v, \quad \text { for every } v \in \chi_{C}^{*}(V), V \in \mathrm{L}(X, Y) .
$$

Let $V_{0} \in \partial \chi_{C}(x)$, or equivalently, $\left\langle V_{0}, x\right\rangle_{Y}-\chi_{C}(x) \in \chi_{C}^{*}\left(V_{0}\right)$. Inserting these elements in the above inequality yields

$$
-\langle F x, x\rangle_{Y}-\chi_{C}(x) \notin \chi_{C}^{*}(-F x)-\operatorname{int} K .
$$

Finally, the domination property implies $-\langle F x, x\rangle_{Y}-\chi_{C}(x) \in \chi_{C}^{*}(-F x)$. Consequently, $x \in C$ is a solution of problem (2.1); compare the first part of this proof. The proof is complete.

Remark 7.2. Some applications of the above inverse results for problem (2.1) can be found in [73, Section 5]. 
Example 7.1. Let us consider Example 2.2 once again. It is easily seen that the mapping $F: \mathbb{R}^{l} \rightarrow$ $\operatorname{Mat}_{k \times l}(\mathbb{R})$, given by relation (2.4), is injective. Thus, the adjoint mapping $F^{-}: \operatorname{Mat}_{k \times l}(\mathbb{R}) \rightarrow \mathbb{R}^{l}$ is defined by $F^{-} U=F^{-1}(-U)$, for every $U \in D\left(F^{-}\right)$, where

$$
D\left(F^{-}\right)=\left\{U \in \operatorname{Mat}_{k \times l}(\mathbb{R}) \mid \text { there is } x \in \mathbb{R}^{l} \text { such that } U=\left(a^{1}-x, \ldots, a^{k}-x\right)^{\top}\right\} .
$$

Therefore, the first inverse vector variational inequality for problem (2.3) consists of finding a matrix $U=\left(a^{1}-x, \ldots, a^{k}-x\right)^{\top} \in D\left(F^{-}\right)$, where $x \in \mathbb{R}^{l}$, such that

$$
\langle V-U,-x\rangle_{Y} \aleph_{\mathrm{int} \mathbb{R}_{\geq}^{k}}^{1} \chi_{\mathbb{R}^{l}}^{*}(U)-\chi_{\mathbb{R}^{l}}^{*}(V), \quad \text { for every } \quad V \in \operatorname{Mat}_{k \times l}(\mathbb{R}) \text { with } \chi_{\mathbb{R}^{l}}^{*}(V) \neq \emptyset .
$$

Note that $\chi_{\mathbb{R}^{l}} \equiv 0$ is the zero mapping. An easy calculation shows that it holds $\chi_{\mathbb{R}^{l}}^{*}(U)=\mathbb{R}^{l}$ if and only if $U \in \operatorname{Mat}_{k \times l}(\mathbb{R})$ has a zero-row and $\chi_{\mathbb{R}^{l}}^{*}(U)=\emptyset$ else. Therefore, problem (7.3) may be written as: find a matrix $U=\left(a^{1}-x, \ldots, a^{k}-x\right)^{\top} \in D\left(F^{-}\right)$, where $x \in \mathbb{R}^{l}$, such that

$$
\langle V-U,-x\rangle_{Y} \aleph_{\mathrm{int} \mathbb{R}_{\geq}^{k}}^{1} \mathbb{R}^{l} \quad \text { for every } \quad V \in \operatorname{Mat}_{k \times l}(\mathbb{R}) \text { with zero-row. }
$$

Recall that the solution set $\mathrm{S}$ of problem (2.3) is equivalent to the convex hull of $a^{1}, \ldots, a^{k}$; compare, for instance, Example 3.1. Consequently, by Theorem 7.1 (i), any matrix $U=\left(a^{1}-x, \ldots, a^{k}-x\right)^{\top} \in D\left(F^{-}\right)$, where $x \in \operatorname{conv}\left\{a^{1}, \ldots, a^{k}\right\}$, is a solution of problem (7.3), which can easily be confirmed.

In a similar way, the second inverse vector variational inequality for problem (2.3) consists of finding a matrix $U=\left(a^{1}-x, \ldots, a^{k}-x\right)^{\top} \in D\left(F^{-}\right)$, where $x \in \mathbb{R}^{l}$, such that

$$
\langle V-U,-x\rangle_{Y} \aleph_{\mathrm{int} \mathbb{R}_{\geq}^{k}}^{2} \chi_{\mathbb{R}^{l}}^{*}(U)-\chi_{\mathbb{R}^{l}}^{*}(V), \quad \text { for every } \quad V \in \operatorname{Mat}_{k \times l}(\mathbb{R}) .
$$

Let us define the set

$$
\operatorname{conv}^{\circ}\left\{a^{1}, \ldots, a^{k}\right\}:=\operatorname{conv}\left\{a^{1}, \ldots, a^{k}\right\} \backslash\left\{a^{1}, \ldots, a^{k}\right\} .
$$

It is easily seen that the sets $A(x):=\left\{-\langle F x, y\rangle_{\mathbb{R}^{k}} \mid y \in \mathbb{R}^{l}\right\}$ and $B(x):=\left\{-\langle F x, x\rangle_{\mathbb{R}^{k}}\right\}$ satisfy the domination property, provided $x \in \operatorname{conv}^{\circ}\left\{a^{1}, \ldots, a^{k}\right\}$. By using the conventions $\emptyset-A:=\emptyset$ and $A \aleph_{\text {int } \mathbb{R}_{\geq}^{k}} \emptyset$ for every subset $A$ of $\mathbb{R}^{k}$, see Section 2, it follows that any matrix $\left(a^{1}-x, \ldots, a^{k}-x\right)^{\top} \in D\left(F^{-}\right)$, where $x \in \operatorname{conv}^{\circ}\left\{a^{1}, \ldots, a^{k}\right\}$, is a solution of problem (7.4). Finally, Theorem 7.1 (ii) implies that any point in $\operatorname{conv}^{\circ}\left\{a^{1}, \ldots, a^{k}\right\}$ is a solution of the vector variational inequality (2.3). In other words, we have shown that it holds

$$
\operatorname{conv}\left\{a^{1}, \ldots, a^{k}\right\} \backslash\left\{a^{1}, \ldots, a^{k}\right\} \subseteq \mathrm{S}
$$

compare the next figure.

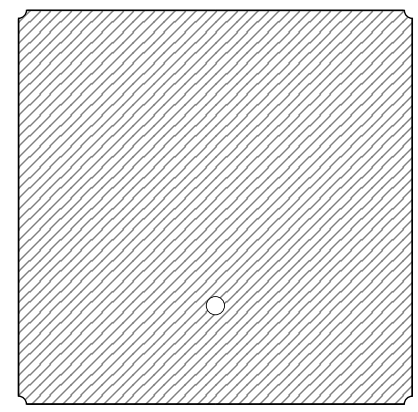

FIGURE 8. Illustration of conv $\left\{a^{1}, \ldots, a^{k}\right\} \backslash\left\{a^{1}, \ldots, a^{k}\right\}$ for the special case $l=2, k=5$ using the data of Example 3.1. 


\section{GAP FUNCTIONS}

This section is devoted to the study of single-valued and set-valued gap functions for vector variational inequality (2.1). The concept of gap functions has been first introduced in the context of optimization and variational problems [16] and subsequently extended among other things to the field of vector variational inequalities. To the best of our knowledge, the introduction of gap functions for vector variational inequalities has been first proposed in $[39,54]$. Since then, there has been a considerable research effort in the study of gap functions for generalized vector variational inequalities and related problems; see, for example, $[5,33,46,48,114,158,159,160]$ and the references therein. By means of a single-valued gap function it is possible to convert vector variational inequality (2.1) into an equivalent optimization problem. To be precise, if $g: X \rightarrow \mathbb{R} \cup\{+\infty\}$ is a single-valued gap function for problem (2.1), compare Definition 8.1, then $x \in C$ is a solution of the vector variational inequality (2.1) if and only if

$$
g(x)=\min _{y \in C} g(y) .
$$

From the computational point of view, such a transformation allows one to apply powerful optimization methods and algorithms for finding solutions of the vector variational inequality. Besides that, gap functions play a fundamental role for developing error bounds for vector variational inequalities. Error bounds provide an upper estimate of the distance of an arbitrary feasible point to the solution set of the underlying problem, that is,

$$
\operatorname{dist}(x, \operatorname{Sol}(\mathrm{VVI})) \leq c \sqrt{g(x)}, \quad \text { for every } \quad x \in C,
$$

where Sol(VVI) denotes the solution set of problem (2.1) and $c>0$ is a constant that depends upon the data of problem (2.1). Some error bounds obtained in the literature, using mainly the projection operator or image space analysis, can be found in $[33,122,157,159,160]$. Thus, in a natural way, the gap functions provides a measure of the violation of the underlying problem, here a vector variational inequality, at any point of the feasible set $C$. Besides that, Fang et al. use the concept of gap functions to investigate convergence results for the vector variational inequality (2.1) in the sense of PainleveKuratowski; compare Section 4 in [87].

Before any further advancement, we give the definition of single-valued and set-valued gap functions for the vector variational inequality (2.1).

Definition 8.1 ([5, Definition 3.1]). Let assumption (A) hold. A function $g: X \rightarrow \mathbb{R} \cup\{+\infty\}$ is said to be a single-valued gap function for problem (2.1), if it satisfies the following conditions:

(i) $g$ is non-negative on $C$, that is, $g(x) \geq 0$ for every $x \in C$.

(ii) It holds $g(x)=0$ if and only if $x \in C$ solves problem (2.1).

Definition 8.2 ([39, Definition 5]). Let assumption (A) hold. A set-valued mapping $G: X \rightrightarrows Y$ is said to be a set-valued gap function for problem (2.1), if it satisfies the following conditions:

(i) For every $x \in C$ it holds $G(x) \nsubseteq-\operatorname{int} K$.

(ii) It holds $0 \in G(x)$ if and only if $x \in C$ is a solution of problem (2.1).

Remark 8.1. (i) Gap functions are sometimes called merit functions in the literature.

(ii) Single-valued and set-valued gap functions for the finite-dimensional vector variational inequality (2.2) are defined in a similar way to the Definitions 8.1 and 8.2; see [33, 106].

(iii) If a function $g: X \rightarrow \mathbb{R} \cup\{+\infty\}$ satisfies condition (i) in Definition 8.1 and

(ii') It holds $g(x)=0$ implies that $x \in C$ solves problem (2.1).

then $g$ is called a weak single-valued gap function for problem (2.1). Examples and applications of weak gap functions can be found, for example, in [164]. 
Definition 8.3 ([116, Section 2]). Let $A$ be a non-empty subset of the Banach space $Y$ with $A \neq Y$. The function $\Delta_{A}: Y \rightarrow \mathbb{R} \cup\{ \pm \infty\}$, defined by

$$
\Delta_{A}(y):=\inf _{y^{\prime} \in A}\left\|y-y^{\prime}\right\|_{Y}-\inf _{y^{\prime} \in Y \backslash A}\left\|y-y^{\prime}\right\|_{Y}, \quad \text { for every } \quad y \in Y,
$$

is called the oriented distance function (or Hiriart-Urruty function).

The oriented distance functions enjoys several useful properties, which we recall in the following.

Proposition 8.1 ([117]). Let $A$ be a non-empty subset of the Banach space $Y$ with $\operatorname{int} A \neq \emptyset$ and $A \neq Y$. Then it holds:

(i) $\Delta_{A}$ is real-valued and Lipschitz-continuous.

(ii) $\Delta_{A}(y)<0$ for every $y \in \operatorname{int} A$.

(iii) $\Delta_{A}(y)=0$ for every $y \in \operatorname{bd} A$.

(iv) $\Delta_{A}(y)>0$ for every $y \in Y \backslash \operatorname{int} A$.

(v) If $A$ is a proper, closed, convex and solid cone in $Y$, then $y-x \in A$ implies $\Delta_{A}(y) \leq \Delta_{A}(x)$.

The following result is Theorem 3.1 in [5] and provides a set-valued gap function for problem (2.1).

Theorem 8.1. Let assumption (A) hold and define the set $B^{*}:=\left\{s \in K^{*} \mid\|s\|_{Y^{*}}=1\right\}$. Then, $g: X \rightarrow$ $\mathbb{R} \cup\{+\infty\}$, defined by

$$
g(x):=\sup _{y \in C} \inf _{s \in B^{*}}\langle s \circ F x, x-y\rangle, \quad \text { for every } \quad x \in X,
$$

is a single-valued gap function for the vector variational inequality (2.1).

Proof. Let $x \in C$ be arbitrarily chosen. The non-negativity of $g$ follows from the estimate

$$
g(x)=\sup _{y \in C} \inf _{s \in B^{*}}\langle s \circ F x, x-y\rangle \geq \inf _{s \in B^{*}}\langle s \circ F x, x-x\rangle=0 .
$$

In order to show the remaining part, note that we have for every $x \in X$

$$
g(x)=-\inf _{y \in C} \Delta_{K}\left(\langle F x, x-y\rangle_{Y}\right) ;
$$

compare Section 3 in [5]. Recall that $\Delta_{K}$ enjoys the representability condition $\Delta_{K}(y)<0$ for $y \in \operatorname{int} K$; compare Proposition 8.1. Thus, for $x \in C$ with $g(x)<0$ there is some $y \in C$ such that

$$
\Delta_{K}\left(\langle F x, x-y\rangle_{Y}\right)<0,
$$

or equivalently, $\langle F x, y-x\rangle_{Y} \in-\operatorname{int} K$. The proof is complete.

The following theorem provides a set-valued gap function for problem (2.1). Note that in [48] the authors discuss differential and sensitivity properties of the set-valued gap function $G$ below. Under suitable conditions, $\mathrm{Li}$ et al. derive expressions for the contingent derivative of $G$; see [48, Sections 3-5].

Theorem 8.2 ([39, Theorem 1]). Let assumption (A) hold. Then, $G: X \rightrightarrows Y$ defined by

$$
G(x):=\mathrm{WMax}\left(\bigcup_{y \in C}\left\{\langle F x, x-y\rangle_{Y}\right\}, K\right), \quad \text { for every } x \in X,
$$

is a set-valued gap function for the vector variational inequality (2.1).

Proof. Let $x \in C$ and $z \in G(x)$. Thus, we have $z \not Z_{\text {int } K}\langle F x, x-y\rangle_{Y}$ for every $y \in C$. In particular, $z \notin-\operatorname{int} K$. For the second part let $x \in C$ such that $0 \in G(x)$. This is equivalent to $\langle F x, x-y\rangle_{Y} \Varangle_{\text {int } K} 0$ for every $y \in C$, that is, $x \in \operatorname{Sol}(\mathrm{VVI})$. Conversely, assume that $x \in C$ is a solution of problem (2.1). We then have

$$
\langle F x, x-y\rangle_{Y} \Varangle_{\text {int } K}\langle F x, x-x\rangle_{Y}
$$

for every $y \in C$, or equivalently $0 \in G(x)$. The proof is complete. 
Example 8.1. Let us consider Example 2.2 again. Thus, the set-valued mapping $G: \mathbb{R}^{l} \rightrightarrows \mathbb{R}^{k}$ in Theorem 8.2 reads

$$
G(x):=\mathrm{WMax}\left(\bigcup_{y \in \mathbb{R}^{k}}\left\{\langle F x, x-y\rangle_{\mathbb{R}^{k}}\right\}, \mathbb{R}_{\geq}^{k}\right), \quad \text { for every } \quad x \in \mathbb{R}^{k}
$$

It should be noted that solving the problem

$$
x \in \mathbb{R}^{l}: \quad 0 \in G(x)
$$

is equally difficult as solving the vector variational inequality (2.2), since we have $0 \in G(x)$ if and only if $x \in \mathbb{R}^{l}$ satisfies

$$
\langle F x, x-y\rangle \Varangle_{\operatorname{int} \mathbb{R}_{\geq}^{k}} 0, \quad \text { for every } \quad y \in \mathbb{R}^{l} .
$$

The following result provides a gap function for problem (2.2) and can also be found in [35]. Note that, in contrast to Theorem 8.1, the gap function below does not involve any scalarization vector.

Theorem 8.3 ([33, Theorem 3.1]). Let assumption (A) hold. The function $g: \mathbb{R}^{l} \rightarrow \mathbb{R} \cup\{+\infty\}$, defined by

$$
g(x):=\sup _{y \in C} \min _{1 \leq j \leq k}\left\langle F_{j} x, x-y\right\rangle, \quad \text { for every } \quad x \in \mathbb{R}^{l},
$$

is a single-valued gap function for the finite-dimensional vector variational inequality (2.2).

Proof. It is easily seen that $g$ is non-negative on $C$ since we have for every $x \in C$

$$
g(x)=\sup _{y \in C} \min _{1 \leq j \leq k}\left\langle F_{j} x, x-y\right\rangle \geq \min _{1 \leq j \leq k}\left\langle F_{j} x, x-x\right\rangle=0 .
$$

Now let $x \in C$ such that $g(x)=0$. Then there exists an index $j \in\{1, \ldots, n\}$ such that $\left\langle F_{j} x, x-y\right\rangle \leq 0$ for every $y \in C$. Consequently, $x$ solves problem (2.2). Conversely, let $x \in C$ be a solution of problem (2.2). Then, we can find an index $j \in\{1, \ldots, n\}$ such that $\left\langle F_{j} x, y-x\right\rangle \geq 0$ for every $y \in C$, implying

$$
\min _{1 \leq j \leq k}\left\langle F_{j} x, x-y\right\rangle \leq 0, \quad \text { for every } \quad y \in C
$$

Thus, $g(x) \leq 0$, and the non-negativity of $g$ implies $g(x)=0$. The proof is complete.

Example 8.2. Let us again come back to Example 2.2. The gap function for problem (2.3), proposed in Theorem 8.3, then becomes

$$
g(x)=\sup _{y \in \mathbb{R}^{l}} \min _{1 \leq j \leq k}\left\langle x-a^{j}, x-y\right\rangle, \quad \text { for every } \quad x \in \mathbb{R}^{l} .
$$

It is not hard to check that it holds $g(x)=0$ if and only if $x \in \operatorname{conv}\left\{a^{1}, \ldots, a^{k}\right\}$. This result again confirms that the solution set $\mathrm{S}$ of problem (2.3) is given by the convex hull of $a^{1}, \ldots, a^{k}$; compare, for example, Example 3.1. Evidently, since the constraining set in problem (2.3) is the whole space $\mathbb{R}^{l}$, we have $g=\chi_{\mathrm{S}}$, where $\chi_{\mathrm{S}}$ denotes the indicator mapping of the set $\mathrm{S}$. 


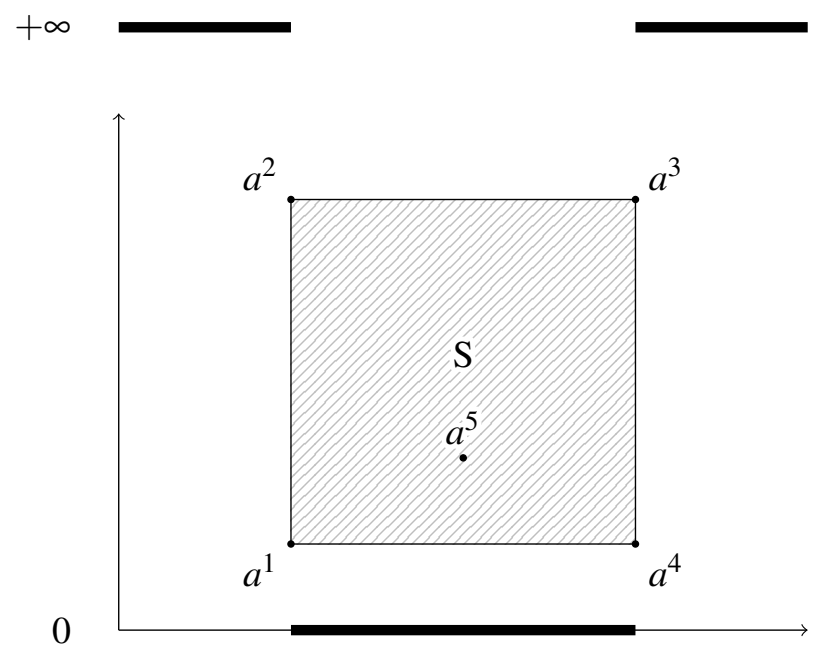

FIGURE 9. Illustration of the single-valued gap function $g: \mathbb{R}^{l} \rightarrow \mathbb{R} \cup\{+\infty\}$ (bold lines) for $l=2$ and $k=5$.

The following theorem provides an error bound result for problem (2.2). We therefore define for $\lambda>0$ the function $g_{\lambda}: \mathbb{R}^{l} \rightarrow \mathbb{R} \cup\{+\infty\}$ by

$$
g_{\lambda}(x):=\sup _{y \in C}\left\{\min _{1 \leq j \leq k}\left\langle F_{j} x, x-y\right\rangle-\frac{\lambda}{2}\|x-y\|_{2}^{2}\right\}, \quad \text { for every } \quad x \in \mathbb{R}^{l} .
$$

It is easy to check that $g_{\lambda}$ is a single-valued gap function for (2.2), which is finite on $C$, compare Section 4 in [35]. In what follows, $\operatorname{dist}(x, A):=\inf _{a \in A}\|x-a\|_{2}$ denotes the distance between the non-empty set $A \subseteq \mathbb{R}^{l}$ and the point $x \in \mathbb{R}^{l}$ with respect to the Euclidean norm $\|\cdot\|_{2}$.

Theorem 8.4 ([33, Theorem 3.3]). Besides assumption (A), assume that the components of $F$ are strongly monotone with the modulus $\mu_{j}>0$, that is, for $j=1, \ldots, k$ it holds that

$$
\left\langle F_{j} x-F_{j} y, x-y\right\rangle \geq \mu_{j}\|x-y\|_{2}^{2}, \quad \text { for every } \quad x, y \in C .
$$

If the intersection $\bigcap_{j=1}^{k} \operatorname{Sol}\left(\mathrm{VI}_{\mathrm{e}_{j}}^{\prime}\right)$ is non-empty and it holds $\mu-\frac{\lambda}{2}>0$, where $\mu:=\min _{1 \leq j \leq k} \mu_{j}$, then

$$
\operatorname{dist}\left(x, \operatorname{Sol}\left(\mathrm{VVI}^{\prime}\right)\right) \leq \frac{1}{\sqrt{\mu-\frac{\lambda}{2}}} \sqrt{g_{\lambda}(x)}, \quad \text { for every } \quad x \in C .
$$

Proof. Let $x \in C$ and $\tilde{x} \in \bigcap_{j=1}^{k} \operatorname{Sol}\left(\mathrm{VI}_{\mathrm{e}_{j}}^{\prime}\right)$ be arbitrarily chosen. Then it holds

$$
g_{\lambda}(x) \geq \min _{1 \leq j \leq k}\left\langle F_{j} x, x-\tilde{x}\right\rangle-\frac{\lambda}{2}\|x-\tilde{x}\|_{2}^{2},
$$

and consequently, using the fact that the components of $F$ are strongly monotone,

$$
g_{\lambda}(x) \geq\left\langle F_{k} \tilde{x}, x-\tilde{x}\right\rangle-\left(\mu-\frac{\lambda}{2}\right)\|x-\tilde{x}\|_{2}^{2},
$$

where we assume without loss of generality that $\left\langle F_{k} x, x-\tilde{x}\right\rangle=\min _{1 \leq j \leq k}\left\langle F_{j} x, x-\tilde{x}\right\rangle$. Since $\tilde{x} \in \operatorname{Sol}\left(\mathrm{VI}_{\mathrm{e}_{k}}^{\prime}\right)$ in particular, it finally follows

$$
g_{\lambda}(x) \geq\left(\mu-\frac{\lambda}{2}\right)\|x-\tilde{x}\|_{2}^{2}
$$

Rearranging the previous inequality and passing to the infimum completes the proof. 
Remark 8.2. Since the assumption

$$
\bigcap_{j=1}^{k} \operatorname{Sol}\left(\mathrm{VI}_{\mathrm{e}_{j}}^{\prime}\right) \neq \emptyset
$$

in the previous theorem is very strong, Charitha et al. replaced it by some geometrical conditions; see Theorem 4.4 in [35].

\section{IMAGE SPACE ANALYSIS}

This section is devoted to the introduction of image space analysis for the following finite-dimensional vector variational inequality: find an element $x \in C$ such that

$$
\langle F x, f(y)-f(x)\rangle_{\mathbb{R}^{k}} \notin-\operatorname{int} \mathbb{R}_{\geq}^{k}, \quad \text { for every } \quad y \in C .
$$

In the above, $F: \mathbb{R}^{l} \rightarrow \operatorname{Mat}_{k \times l}(\mathbb{R})$ and $f: \mathbb{R}^{l} \rightarrow \mathbb{R}^{l}$ are given mappings. The non-empty constraining set $C$ is defined by $C:=\left\{x \in \mathbb{R}^{l} \mid g(x) \in \mathbb{R}_{\geq}^{m}\right\}$, where $g: \mathbb{R}^{l} \rightarrow \mathbb{R}^{m}$ is a mapping with $g\left[\mathbb{R}^{l}\right] \cap \mathbb{R}_{\geq}^{m} \neq \emptyset$. The development of image space analysis has a very long history, while traces of the idea of studying the images of functions involved in optimization problems go back to the work of Carathéodory; see [29]. The basic idea of image space analysis, which has been proposed in [100] for the first time, is quite analogous to that, which led from Riemann integral to Lebesgue one: instead of performing the analysis in the space, where problem (9.1) is given, image space analysis does it in the space, where the images of the functions involved in problem (9.1) run. Evidently, an element $x \in C$ is a solution of problem (9.1) if and only if there exists no $y \in \mathbb{R}^{l}$ such that

$$
\langle F x, f(y)-f(x)\rangle_{\mathbb{R}^{k}} \in-\operatorname{int} \mathbb{R}_{\geq}^{k}, \quad g(y) \in \mathbb{R}_{\geq}^{m} .
$$

In other words, the following system in the unknown $y$ is impossible:

$$
u=\langle F x, f(x)-f(y)\rangle_{\mathbb{R}^{k}} \in \operatorname{int} \mathbb{R}_{\geq}^{k}, \quad v=g(y) \in \mathbb{R}_{\geq}^{m}, \quad y \in \mathbb{R}^{l} .
$$

The space $\mathbb{R}^{k} \times \mathbb{R}^{m}$, where the new variable $(u, v)$ runs, is called the image space associated with problem (9.1). By introducing the sets

$$
\mathscr{H}:=\operatorname{int} \mathbb{R}_{\geq}^{k} \times \mathbb{R}_{\geq}^{m} \text { and } \mathscr{K}(x):=\left\{(u, v) \in \mathbb{R}^{k} \times \mathbb{R}^{m} \mid u=\langle F x, f(x)-f(y)\rangle_{\mathbb{R}^{k}}, v=g(y), y \in \mathbb{R}^{l}\right\},
$$

we can claim that $x \in C$ is a solution of the vector variational inequality (9.1) if and only if

$$
\mathscr{H} \cap \mathscr{K}(x)=\emptyset ;
$$

compare $[100,104]$. To perform image space analysis, we can indifferently begin with system (9.2) or (9.3). The formulation (9.3) corresponds to adopt an algebraic and geometric language, which will lead us to use separation theorems, while (9.2) leads to adopt so-called theorems of the alternatives. By using (9.3), we introduce the following equivalent image problem: given $x \in \mathbb{R}^{l}$, find an element $(\tilde{u}, \tilde{v}) \in \mathscr{K}(x) \cap\left(\mathbb{R}^{k} \times \mathbb{R}_{\geq}^{m}\right)$ such that

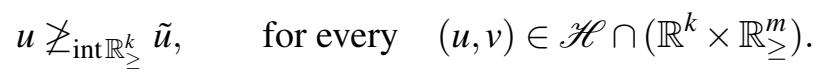

As we can see, in the image space we define an optimization problem also when in the initial space we are dealing with a vector variational inequality. This is because problem (9.1) is equivalent to the impossibility of the system (9.2). It should be further noted that image space analysis may be applied to any kind of problem, which can be expressed under the form of the inconsistency of a parametric system; see [100]. These may include, for example, constrained optimization problems, variational inequalities, equilibrium problems, and numerous others; see [43, 44, 50, 100, 168, 187] and the references therein. In the context of vector variational inequalities, theorems of the alternative and seperation results have 
turned out to be a powerful tool for deriving necessary and sufficient optimality conditions; see [50]. Therefore, the authors in [44] proposed to study

$$
\mathscr{M}(x) \cap \mathscr{N}(x)=\emptyset
$$

instead of (9.3), where

$$
\begin{aligned}
& \mathscr{M}(x):=\left(\mathbb{R}^{l} \backslash \mathscr{C}(x)\right) \times \mathbb{R}_{\geq}^{m} \times \mathbb{R}_{\geq}^{m}, \\
& \mathscr{N}(x):=\left\{(u, v, w) \in \mathbb{R}^{l+2 m} \mid u=f(y)-f(x), v=g(y), w=g(x), y \in \mathbb{R}^{l}\right\},
\end{aligned}
$$

and $\mathscr{C}(x):=\left\{z \in \mathbb{R}^{l} \mid\langle F x, z\rangle_{\mathbb{R}^{k}} \notin-\operatorname{int} \mathbb{R}_{\geq}^{k}\right\}$, for every $x \in \mathbb{R}^{l}$. Evidently, (9.4) holds if and only if $x \in C$ is a solution of problem (9.1). This reformulation allows to relate the vector variational inequality (9.1) to a multi-objective optimization problem [44, Section 3.1] and to derive efficient tools for solving problem (9.1).

Remark 9.1. (i) Vector variational inequalities of the form (9.1) are frequently called inverse vector variational inequalities; see, for example, [43, 44]. However, it should be noted that the structure of problem (9.1) is different from that of the problems (7.1) and (7.2), respectively.

(ii) By introducing the set $\Omega:=\left\{f(x) \mid x \in \mathbb{R}^{l}, g(x) \in \mathbb{R}_{\geq}^{m}\right\}$, problem (9.1) is frequently stated in the following [44] equivalent way: find an element $x \in \mathbb{R}^{l}$ such that

$$
f(x) \in \Omega \quad \text { and } \quad\left\langle F x, f_{\Omega}-f(x)\right\rangle_{\mathbb{R}^{k}} \not_{\mathrm{int} \mathbb{R}_{\geq}^{k}} 0, \quad \text { for every } \quad f_{\Omega} \in \Omega .
$$

(iii) Evidently, if $f: \mathbb{R}^{l} \rightarrow \mathbb{R}^{l}$ is the identity mapping, then problem (9.1) recovers the vector variational inequality (2.1).

9.1. Optimality conditions. The aim of this section is to give necessary and sufficient optimality conditions for problem (9.1). In what follows, we will consider the multi-objective-like optimization problem

$$
\operatorname{Eff}(f[C], \mathscr{C}(\cdot)) \text {. }
$$

Here we say that $x \in C$ is an efficient solution of problem (9.5) if and only if $f(y)-f(x) \notin-\mathscr{C}(x) \backslash\{0\}$ for all $y \in C$.

The next lemma shows that problem (9.5) is necessary for the vector variational inequality (9.1).

Lemma 9.1. Any solution $x \in C$ of problem (9.5) with $\mathscr{C}(x) \cup(-\mathscr{C}(x))=\mathbb{R}^{l}$ is a solution of the vector variational inequality (9.1).

Proof. Let $x \in C$ be a solution of problem (9.5). Since it holds $\mathscr{C}(x) \cup(-\mathscr{C}(x))=\mathbb{R}^{l}$, we deduce $f(y)-$ $f(x) \in \mathscr{C}(x)$ for every $y \in C$. Hence, $x \in C$ solves the vector variational inequality (9.1). The proof is complete.

Remark 9.2. A proper cone $K$ in $\mathbb{R}^{l}$ with $K \cup(-K)=\mathbb{R}^{l}$ is frequently called connected; see [189, Section 4].

Proposition 9.1 ([44, Proposition 3.1]). Let $x \in C$ be a solution of problem $(9.5)$ with $\mathscr{C}(x) \cup(-\mathscr{C}(x))=$ $\mathbb{R}^{l}$. Then it holds

$$
\sup _{y \in C} \Delta_{\mathscr{C}(x)}(f(y)-f(x))=0 .
$$

Proof. Let $x \in C$ be a solution of problem (9.5) such with $\mathscr{C}(x) \cup(-\mathscr{C}(x))=\mathbb{R}^{l}$. Thus, $f(y)-f(x) \in$ $\mathscr{C}(x)$ for every $y \in C$; see Lemma 9.1. Therefore, in view of Proposition 8.1 (ii) and (iii), we have

$$
\sup _{y \in C} \Delta_{\mathscr{C}(x)}(f(y)-f(x)) \leq 0 .
$$


However, equality holds due to $0 \in \operatorname{bd} \mathscr{C}(x)$ and the estimate

$$
\sup _{y \in C} \Delta_{\mathscr{C}(x)}(f(y)-f(x)) \geq \Delta_{\mathscr{C}(x)}(f(x)-f(x))=\Delta_{\mathscr{C}(x)}(0)=0 ;
$$

compare again Proposition 8.1 (iii). The proof is complete.

Theorem 9.1 ([44, Theorem 3.1]). An element $x \in C$ is a solution of the vector variational inequality (9.1) if and only if relation (9.6) holds.

Proof. Let $x \in C$ be a solution of problem (9.1). In other words, it holds $f(y)-f(x) \in \mathscr{C}(x)$ for every $y \in C$. Following the proof of Proposition 9.1, we conclude that (9.6) holds. Conversely, assume that (9.6) holds. Then we have

$$
\Delta_{\mathscr{C}(x)}(f(y)-f(x)) \leq 0, \quad \text { for every } \quad y \in C,
$$

in particular. This together with Proposition 8.1 (ii) and (iii) yields $f(y)-f(x) \in \mathscr{C}(x)$ for every $y \in C$. Consequently, $x \in C$ is a solution of the vector variational inequality (9.1) and the proof is complete.

9.2. Separation functions. The purpose of this section is to recall the concept of separation functions for problem (9.1). As already mentioned, an element $x \in C$ is a solution of the vector variational inequality (9.1) if and only if relation (9.4) holds. Evidently, this is equivalent to saying that the following system in the unknown $y$ is impossible:

$$
f(y)-f(x) \in \mathbb{R}^{l} \backslash \mathscr{C}(x), \quad g(x) \in \mathbb{R}_{\geq}^{m}, \quad g(y) \in \mathbb{R}_{\geq}^{m}, \quad y \in \mathbb{R}^{l} .
$$

It should be noted that, using the above concept, the image space associated with the finite-dimensional vector variational inequality (9.1) is $\mathbb{R}^{l} \times \mathbb{R}^{m} \times \mathbb{R}^{m}=\mathbb{R}^{l+2 m}$.

In what follows, we give the definitions of weak and regular separation functions. Recall that the level sets $l e v>\psi$ and $l e_{\geq} \psi$ of a mapping $\psi: \mathbb{R}^{l+2 m} \rightarrow \mathbb{R} \cup\{ \pm \infty\}$ are defined by lev $>\psi:=\left\{x \in \mathbb{R}^{l+2 m} \mid\right.$ $\psi(x)>0\}$ and $\operatorname{lev}_{\geq} \psi:=\left\{x \in \mathbb{R}^{l+2 m} \mid \psi(x) \geq 0\right\}$, respectively.

Definition 9.1 ([50, Definition 2.1]). Let $x \in \mathbb{R}^{l}$ and let $\Lambda$ be a non-empty subset of $\mathbb{R}$. A class of functions $\omega: \mathbb{R}^{l+2 m} \times \Lambda \rightarrow \mathbb{R} \cup\{ \pm \infty\}$, such that

(i) $\operatorname{lev}_{\geq} \omega(\cdot, \lambda) \supseteq \mathscr{M}(x)$, for every $\lambda \in \Lambda$,

(ii) $\bigcap_{\lambda \in \Lambda} l_{\text {ev }} \omega(\cdot, \lambda) \subseteq \mathscr{M}(x)$,

is called the class of weak separation functions and is denoted by $\mathrm{W}(x, \Lambda)$.

Definition 9.2 ([50, Definition 2.2]). Let $x \in \mathbb{R}^{l}$ and let $\Lambda$ be a non-empty subset of $\mathbb{R}$. A class of functions $\omega: \mathbb{R}^{l+2 m} \times \Lambda \rightarrow \mathbb{R} \cup\{ \pm \infty\}$, such that

$$
\bigcap_{\lambda \in \Lambda} \operatorname{lev}_{>} \omega(\cdot ; \lambda)=\mathscr{M}(x),
$$

is called the class of regular weak separation functions and is denoted by $\operatorname{RW}(x, \Lambda)$.

In what follows, we are going to construct a class of weak and regular weak separation functions, respectively; see [44, Section 3.2]. For this purpose, we denote by Proj: $\mathbb{R}^{2 m} \rightarrow \mathbb{R}^{2 m}$ the orthogonal projection onto $\mathbb{R}_{\geq}^{2 m}=\mathbb{R}_{\geq}^{m} \times \mathbb{R}_{\geq}^{m}$, satisfying

$$
\|(v, z)-\operatorname{Proj}(v, z)\|_{2}=\inf _{(\tilde{v}, \tilde{z}) \in \mathbb{R}_{\geq}^{2 m}}\|(v, z)-(\tilde{v}, \tilde{z})\|_{2}, \quad \text { for every } \quad(v, z) \in \mathbb{R}_{\geq}^{2 m} .
$$

Thus, given $x \in \mathbb{R}^{l}$, we consider a family of non-linear functions $\omega: \mathbb{R}^{l+2 m} \times \mathbb{R} \rightarrow \mathbb{R} \cup\{ \pm \infty\}$ given by

$$
\omega(u, v, z, \lambda):=-\lambda \Delta_{\mathbb{R}^{l} \backslash \mathscr{C}(x)}(u)-\|(v, z)-\operatorname{Proj}(v, z)\|_{2}, \quad \text { for every } \quad(u, v, z, \lambda) \in \mathbb{R}^{l+2 m} \times \mathbb{R}_{\geq} ;
$$

compare [44]. Evidently, for every $\lambda \in \mathbb{R}_{\geq}, \omega(\cdot, \lambda)$ is lipschitz continuous in $\mathbb{R}^{l+2 m}$. Further, since $\mathscr{C}(x)$ is non-empty, $\omega(\cdot, \lambda)$ is finite-valued. 
The next proposition shows that (9.8) defines a family of weak and regular separation functions, respectively.

Proposition 9.2 ([44, Proposition 3.2]). Let $x \in \mathbb{R}^{l}$ be arbitrarily chosen and assume that the class of functions $\omega: \mathbb{R}^{l+2 m} \times \mathbb{R} \rightarrow \mathbb{R} \cup\{ \pm \infty\}$ is given by (9.8). Then it holds $\omega \in \mathrm{W}\left(x, \mathbb{R}_{\geq}\right)$and $\omega \in \mathrm{RW}\left(x, \mathbb{R}_{>}\right)$.

Proof. Let $x \in \mathbb{R}^{l}$. We show $\omega \in \mathrm{W}\left(x, \mathbb{R}_{\geq}\right)$only since the second assertion of this proposition follows in a similar fashion. In order to show

$$
\operatorname{lev}_{\geq} \omega(\cdot, \lambda) \supseteq \mathscr{M}(x), \quad \text { for every } \quad \lambda \in \mathbb{R}_{\geq}
$$

let $(u, v, z) \in \mathscr{M}(x)$ and $\lambda \in \mathbb{R}_{\geq}$. Let us show that it holds $\omega(u, v, z, \lambda) \geq 0$. Since $\mathscr{C}(x)$ is a closed cone, we have $\mathbb{R}^{l} \backslash \mathscr{C}(x)=\operatorname{int}\left(\mathbb{R}^{l} \backslash \mathscr{C}(x)\right)$. In view of Proposition 8.1 (ii), we consequently have $-\lambda \Delta_{\mathbb{R}^{l} \backslash \mathscr{C}(x)}(u)>0$. Further, it trivially holds $\|(v, z)-\operatorname{Proj}(v, z)\|_{2}=0$ since $(v, z) \in \mathbb{R}_{\geq}^{2 m}$. This implies

$$
\omega(u, v, z, \lambda)=-\lambda \Delta_{\mathbb{R}^{l} \backslash \mathscr{C}(x)}(u)-\|(v, z)-\operatorname{Proj}(v, z)\|_{2}=-\lambda \Delta_{\mathbb{R}^{l} \backslash \mathscr{C}(x)}(u)>0,
$$

which shows (9.9). Evidently, we have

$$
\omega(u, v, z, 0)=-\|(v, z)-\operatorname{Proj}(v, z)\|_{2} \leq 0, \quad \text { for every } \quad(u, v, z) \in \mathbb{R}^{l+2 m},
$$

and therefore $\operatorname{lev}_{\geq} \omega(\cdot, 0)=\emptyset$. Consequently, it trivially holds

$$
\emptyset=\bigcap_{\lambda \in \mathbb{R}_{\geq}} \operatorname{lev}_{>} \omega(\cdot, \lambda) \subseteq \mathscr{M}(x) .
$$

This shows $\omega \in \mathrm{W}\left(x, \mathbb{R}_{\geq}\right)$, which completes the proof.

We now come to the first theorem of the alternative, which is Theorem 3.3 in [44].

Theorem 9.2. Let $x \in \mathbb{R}^{l}$ be arbitrarily chosen. Then, problem (9.7) in the unknown $y$ and the system

$$
\text { there is } \lambda \in \mathbb{R}_{\geq} \text {such that } \omega(f(y)-f(x), g(y), g(x), \lambda)<0, \quad \text { for every } y \in \mathbb{R}^{l},
$$

where $\omega$ is given by (9.8), are not simultaneously possible.

Proof. Assume first that problem (9.7) is possible. Then we can find $y \in \mathbb{R}^{l}$ such that $f(y)-f(x) \notin \mathscr{C}(x)$, $g(x) \in \mathbb{R}_{\geq}^{m}$ and $g(y) \in \mathbb{R}_{\geq}^{m}$. Moreover, we have

$$
(f(y)-f(x), g(y), g(x)) \in \mathscr{M}(x),
$$

and Proposition 9.2, compare relation (9.9), implies

$$
\omega(f(y)-f(x), g(y), g(x), \lambda) \geq 0, \quad \text { for every } \quad \lambda \in \mathbb{R}_{\geq} .
$$

In other words, system (9.10) is impossible. Conversely, assume that system (9.10) is possible. Then we can find $\lambda \in \mathbb{R}_{\geq}$such that

$$
\mathscr{N}(x) \cap\left\{(u, v, z) \in \mathbb{R}^{l+2 m} \mid \omega(u, v, z, \lambda) \geq 0\right\}=\emptyset .
$$

Since $\mathscr{M}(x) \subseteq \operatorname{lev}_{\geq} \omega(\cdot, \lambda)$, we further have

$$
(f(y)-f(x), g(y), g(x)) \in \mathscr{M}(x), \quad \text { for every } \quad y \in \mathbb{R}^{l} .
$$

In other words, system (9.7) is impossible. The proof is complete. 


\section{Stability AND SENSITIVITY ANALYSiS}

The purpose of this section is to present some stability and sensitivity results for the finite-dimensional vector variational inequality (2.2). It is well-known that variational problems are ill-posed in general, that is, they may either have no solution or multiple solutions, and, most importantly, small errors in data could lead to uncontrollable errors in its solution(s); see [75, 97, 99, 108, 113, 144] and the references therein. In addition to that, the solution of vector variational inequalities is non-unique in general; compare, for instance, Example 2.2. On the other hand, from a practical point of view, one cannot expect that the data of the vector variational inequality (2.2), here the constraining set $C \subseteq \mathbb{R}^{l}$ and the mapping $F: \mathbb{R}^{l} \rightarrow \operatorname{Mat}_{k \times l}(\mathbb{R})$, are known. Due to this incompleteness, many authors have studied problem (2.2) with respect to contaminated/perturbed data, depending on some parameters signifying the level of error in a certain sense; see $[6,49,52,58,85,89,124,162]$ and the references therein. To be precise, let $U$ and $V$ be Banach spaces, the so-called parameter spaces, let $E: U \rightrightarrows \mathbb{R}^{l}$ be a set-valued mapping with non-empty, closed and convex values, and let $\mathscr{F}: V \times \mathbb{R}^{l} \rightarrow \mathrm{Mat}_{k \times l}(\mathbb{R})$ be a mapping. Given parameters $\rho \in U$ and $\eta \in V$, the perturbed finite-dimensional vector variational inequality consists of finding an element $x=x(\rho, \eta) \in E(\rho)$ such that

$$
\langle\mathscr{F}(\eta, x), y-x\rangle_{\mathbb{R}^{k}} \notin-\operatorname{int} \mathbb{R}_{\geq}^{k}, \quad \text { for every } \quad y \in E(\rho) .
$$

Note that problem (10.1) recovers the vector variational inequality (2.2) if $E$ is constant and $\mathscr{F}$ does not depend on the parameter $\eta$. In what follows, we are going to denote the solution set of problem (10.1), depending on the parameters $\rho \in U$ and $\eta \in V$, by $\operatorname{Sol}(\rho, \eta)$. When the parameters $\rho$ and $\eta$ vary in $U$ and $V$, this defines a set-valued mapping Sol $: U \times V \rightrightarrows \mathbb{R}^{l}$ by

$$
\operatorname{Sol}(\rho, \eta):=\left\{x \in E(\rho) \mid\langle\mathscr{F}(\eta, x), y-x\rangle_{\mathbb{R}^{k}} \notin-\operatorname{int} \mathbb{R}_{\geq}^{k}, \text { for every } y \in E(\rho)\right\} .
$$

Within the last years, several authors have investigated the stability analysis of problem (2.2), that is, the qualitative analysis of several properties of the solution mapping Sol $: U \times V \rightrightarrows \mathbb{R}^{l}$. These include, for example, the upper semicontinuity, the lower semicontinuity, the (lipschitz) continuity and the Robinson's metric regularity property of Sol; see $[1,49,52,58,86,89,124]$. In this research area, scalarization techniques [52, 85, 141] and gap functions [47] have proven to be powerful tools. In contrast, the sensitivity analysis of problem (2.2) means the quantitative analysis of the solution mapping. Note that Sol can be formulated [86] as a particular parametric variational system, that is,

$$
\operatorname{Sol}(\rho, \eta)=\{x \in E(\rho) \mid q(\eta, x) \in Q(\rho, \eta, x)\},
$$

where we use the notation $q(\eta, x):=\langle\mathscr{F}(\eta, x), x\rangle_{\mathbb{R}^{k}}$ and $Q(\rho, \eta, x):=\operatorname{WMin}\left(\langle\mathscr{F}(\eta, x), E(\rho)\rangle_{\mathbb{R}^{k}}, \mathbb{R}_{\geq}^{k}\right)$. Here, one main research focus is the study of derivatives of Sol in terms of derivatives of $q$ and $Q$; see [86, Section 4]. Such properties are crucial to derive necessary optimality conditions for problem (2.2). See [56] for globally proper efficiency results and optimality conditions for generalized vector variational inequalities involving the generalized contingent epiderivative. Besides stability and sensitivity analysis, the well-posedness of problem (2.2) and related problems has been investigated in [83, 143]. Generally speaking, it deals with the behavior of the solution with respect to the problem's data pertubations. Note that an initial concept of well-posedness for scalar optimization problems has been introduced in [183] for the first time, known as Tykhonov well-posedness. In addition to the previous research areas related to stability and sensitivity analysis, the Painlevé-Kuratowski upper and lower convergence of perturbed vector variational inequalities has been investigated in [6, 87].

Before any further advancement, we recall continuity notions for set-valued mappings.

Definition 10.1 ([15, 23]). Let $X$ and $Y$ be Banach spaces. A set-valued mapping $S: X \rightrightarrows Y$ is said to be

(i) upper semicontinuous at $x \in X$ if for any open set $V$ in $Y$ containing $S(x)$, there exists an open neighborhood $U$ of $x$ in $X$ such that $S(z) \subseteq V$ for all $z \in U$; 
(ii) lower semicontinuous at $x \in X$ if for any open set $V$ in $Y$ such that $S(x) \cap V \neq \emptyset$, there exists an open neighborhood $U$ of $x$ in $X$ such that $S(z) \cap V \neq \emptyset$ for all $z \in U$;

(iii) upper semicontinuous (respectively, lower semicontinuous) on $X$ if it is upper semicontinuous (respectively, lower semicontinuous) at every point in $X$;

(iv) continuous on $X$ if it is upper semicontinuous and lower semicontinuous;

(v) compact if there exists a compact set $B \subseteq Y$ such that $S(x) \subseteq B$ for every $x \in X$.

Remark 10.1. (i) If the set $S(x)$ is singleton in a neighborhood of $x \in X$, then the upper semicontinuity and lower semicontinuity of $S$ at $x$ are equivalent; see [12, Remark 1.21].

(ii) It should be noted that a set-valued mapping with compact values does not have to be compact in general. For example, consider $X=Y=\mathbb{R}$ and $S(x)=\left[0, x^{2}\right]$ for $x \in \mathbb{R}$.

The next two lemma give handy characterizations of upper and lower semicontinuity.

Lemma 10.1 ([23, Chapter 6, Corollary]). Let $X$ and $Y$ be Banach spaces. A compact set-valued mapping $S: X \rightrightarrows Y$ is upper semicontinuous if and only if the graph of $S$ is a closed subset of $X \times Y$.

Lemma 10.2 ([15, 23]). Let $X$ and $Y$ be Banach spaces. A set-valued mapping $S: X \rightrightarrows Y$ is lower semicontinuous at $x \in X$ if and only if for any sequence $\left\{x_{n}\right\} \subseteq X, x_{n} \rightarrow x$ and for any $y \in S(x)$, there is a sequence $\left\{y_{n}\right\}$ such that $y_{n} \in S\left(x_{n}\right)$ and $y_{n} \rightarrow y$.

Slightly modifying the proof in [85, Theorem 2.2], we have the following result.

Theorem 10.1. Let $\mathscr{F}: V \times \mathbb{R}^{l} \rightarrow \operatorname{Mat}_{k \times l}(\mathbb{R})$ be a given mapping and suppose that the following conditions are satisfied:

(i) The set-valued mapping $E: U \rightrightarrows \mathbb{R}^{l}$ has non-empty, closed and convex values. Further, $E$ is compact and has a closed graph.

(ii) For every $x \in \mathbb{R}^{l}$, the mapping $\mathscr{F}(\cdot, x): V \rightarrow \operatorname{Mat}_{k \times l}(\mathbb{R})$ is continuous.

(iii) For every $\eta \in V$, the mapping $\mathscr{F}(\eta, \cdot): \mathbb{R}^{l} \rightarrow \mathrm{Mat}_{k \times l}(\mathbb{R})$ is $v$-hemicontinuous and $\mathbb{R}_{\geq}^{k}$-monotone. Then, the solution mapping Sol : $U \times V \rightrightarrows \mathbb{R}^{l}$ of problem (10.1) is upper semicontinuous on $U \times V$.

Proof. By assumption (i), we can find a compact set $B \subseteq \mathbb{R}^{l}$ such that $E(\rho) \subseteq B$ for every $\rho \in U$. Evidently, Sol is compact, since it holds $\operatorname{Sol}(\rho, \eta) \subseteq E(\rho)$ for every $\rho \in U$ and $\eta \in V$. Note further that the values of Sol are non-empty; see Theorem 5.1. In order to show that Sol is upper semicontinuous, we are going to use Lemma 10.1. For this purpose, let $\left\{x_{n}\right\} \subseteq \mathbb{R}^{l}$ and $\left\{\left(\rho_{n}, \eta_{n}\right)\right\} \subseteq U \times V$ be sequences with $x_{n} \in \operatorname{Sol}\left(\rho_{n}, \eta_{n}\right), x_{n} \rightarrow x, \rho_{n} \rightarrow \rho$ and $\eta_{n} \rightarrow \eta$. In the following, we are going to show that $x \in \operatorname{Sol}(\rho, \eta)$. Since $x_{n} \in \operatorname{Sol}\left(\rho_{n}, \eta_{n}\right)$, it holds $x_{n} \in E\left(\rho_{n}\right)$ and

$$
\left\langle\mathscr{F}\left(\eta_{n}, x_{n}\right), y-x_{n}\right\rangle_{\mathbb{R}^{k}} \notin-\operatorname{int} \mathbb{R}_{\geq}^{k}, \quad \text { for every } \quad y \in E\left(\rho_{n}\right),
$$

Consequently $x \in E(\rho)$ since $E$ has a closed graph. By assumption (iii), the above inequality is equivalent to

$$
\left\langle\mathscr{F}\left(\eta_{n}, y\right), y-x_{n}\right\rangle_{\mathbb{R}^{k}} \notin-\operatorname{int} \mathbb{R}_{\geq}^{k}, \quad \text { for every } \quad y \in E\left(\rho_{n}\right) ;
$$

see Lemma 4.1. Since $\mathscr{F}$ is assumed to be continuous in the first component, passing in the above inequality to the limit yields

$$
\left\langle\mathscr{F}\left(\eta_{n}, y\right), y-x_{n}\right\rangle_{\mathbb{R}^{k}} \rightarrow\langle F(\eta, y), y-x\rangle_{\mathbb{R}^{k}},
$$

for every $y \in E(\rho)$. Applying Lemma 4.1 once again, we have finally shown that $x \in E(\rho)$ satisfies

$$
\langle\mathscr{F}(\eta, x), y-x\rangle_{\mathbb{R}^{k}} \notin-\operatorname{int} \mathbb{R}_{\geq}^{k}, \quad \text { for every } \quad y \in E(\rho),
$$

or equivalently, $x \in \operatorname{Sol}(\rho, \eta)$. Thus, we have shown that $\operatorname{Sol}$ is upper semicontinuous on $U \times V$, which completes the proof. 
In general, the proof of lower semicontinuity is more challenging [58] than the one of upper semicontinuity. For the sake of simplicity, we therefore consider the following perturbed finite-dimensional vector variational inequality only: given a parameter $\eta \in V$, find an element $x=x(\eta) \in C$ such that

$$
\langle\mathscr{F}(\eta, x), y-x\rangle_{\mathbb{R}^{k}} \notin-\text { int } \mathbb{R}_{\geq}^{k}, \quad \text { for every } \quad y \in C .
$$

In the above, $C$ is a non-empty, closed and convex subset of $\mathbb{R}^{l}$. We again denote the corresponding solution mapping of problem (10.2) by Sol. Note that problem (10.2) is obtained by letting $E(x)=C$, for every $x \in U$, in problem (10.1).

The proof of the following result is based on Theorem 3.1 in [58].

Theorem 10.2. Let $\mathscr{F}: V \times \mathbb{R}^{l} \rightarrow \mathrm{Mat}_{k \times l}(\mathbb{R})$ be a given mapping and suppose that the following conditions are satisfied:

(i) $C$ is a non-empty, convex and compact subset of $\mathbb{R}^{l}$.

(ii) $\mathscr{F}$ is continuous.

(iii) For every $\eta \in V$, the mapping $\mathscr{F}(\eta, \cdot): \mathbb{R}^{l} \rightarrow \operatorname{Mat}_{k \times l}(\mathbb{R})$ is $\mathbb{R}_{\geq}^{k}$-monotone. Further, there exists an index $j \in\{1, \ldots, k\}$ such that for every $\eta \in V$, the jth component $\mathscr{F}_{j}(\eta, \cdot): \mathbb{R}^{l} \rightarrow \mathbb{R}^{l}$ is strictly monotone.

Then, the solution mapping Sol $: V \rightrightarrows \mathbb{R}^{l}$ of problem (10.2) is lower semicontinuous on $V$.

Proof. Let $s \in \mathbb{R}_{\geq}^{k} \backslash\{0\}$. Given a parameter $\eta \in V$, we consider the scalar parametric variational inequality of finding an element $x \in C$ such that

$$
\left\langle\sum_{j=1}^{k} s_{j} \mathscr{F}_{j}(\eta, x), y-x\right\rangle \geq 0, \quad \text { for every } \quad y \in C .
$$

Let us denote the solution set of problem (10.3), depending on $s$ and $\eta$, by $\operatorname{Sol}_{s}(\eta)$. Evidently, $\operatorname{Sol}_{s}(\eta)$ is non-empty for every $\eta \in V$ since $\mathscr{F}$ is continuous and $C$ is non-empty, convex and compact; see Theorem 5.9. As a consequence of assumption (iii), the mapping $\sum_{j=1}^{k} s_{j} \mathscr{F}_{j}(\eta, \cdot): \mathbb{R}^{l} \rightarrow \mathbb{R}^{l}$ is strictly monotone. Thus, problem (10.3) has a unique solution, that is, $\operatorname{Sol}_{s}(\eta)$ is a singleton for every $s \in \mathbb{R}_{\geq}^{k} \backslash\{0\}$ and every $\eta \in V$. Let us show that for fixed $s \in \mathbb{R}_{\geq}^{k} \backslash\{0\}$, the solution mapping $\operatorname{Sol}_{s}: V \rightarrow \mathbb{R}^{l}$ is continuous. Indeed, let $s \in \mathbb{R}_{>}^{k} \backslash\{0\}$ and $\eta \in V$ be arbitrarily chosen and let $\left\{\eta_{n}\right\} \subseteq V$ be a sequence such that $\eta_{n} \rightarrow \eta$. Denote by $x_{n}$ the corresponding unique solution of problem (10.3) with respect to $s$ and $\eta_{n}$, that is, $x_{n}:=\operatorname{Sol}_{s}\left(\eta_{n}\right)$. Since $C$ is compact and $\left\{x_{n}\right\} \subseteq C$, we can find a subsequence, again denoted by $\left\{x_{n}\right\}$, such that $x_{n} \rightarrow x$. We further have

$$
\left\langle\sum_{j=1}^{k} s_{j} \mathscr{F}_{j}\left(\eta_{n}, x_{n}\right), y-x_{n}\right\rangle \geq 0, \quad \text { for every } \quad y \in C .
$$

Due to the continuity of $\mathscr{F}$, we can pass in the above inequality to the limit. Thus, $x \in C$ is the unique solution of problem (10.3), which shows the continuity of $\mathrm{Sol}_{s}$.

In order to prove that the solution mapping Sol $: V \rightrightarrows \mathbb{R}^{l}$ of problem (10.1) is lower semicontinuous on $V$, we are going to use Lemma 10.2. For this purpose let $\left\{\eta_{n}\right\} \subseteq V$ be a sequence such that $\eta_{n} \rightarrow \eta$ and let $x \in \operatorname{Sol}(\eta)$. Similar to Proposition 3.1, it is easy to show that for every $\eta \in V$ it holds that

$$
\operatorname{Sol}(\eta)=\bigcup_{s \in \mathbb{R}_{\geq}^{k} \backslash\{0\}} \operatorname{Sol}_{s}(\eta)
$$

Consequently, there is some $\hat{s} \in \mathbb{R}_{>}^{k} \backslash\{0\}$ such that $x$ is the unique solution of problem (10.3), depending on $\hat{s}$ and $\eta$. In other words, $x=\operatorname{Sol}_{\hat{s}}(\eta)$. Let us define a sequence $\left\{x_{n}\right\}$ in $\mathbb{R}^{l}$ by

$$
x_{n}:=\operatorname{Sol}_{\hat{s}}\left(\eta_{n}\right) \text {. }
$$


Note that we have $x_{n} \in \operatorname{Sol}\left(\eta_{n}\right)$ in particular. Since $\operatorname{Sol}_{\hat{s}}: V \rightarrow \mathbb{R}^{l}$ is continuous, we finally have $x_{n} \rightarrow x$. Thus, the lower semicontinuity of Sol $: V \rightrightarrows \mathbb{R}^{l}$ follows and the proof is complete.

Example 10.1. This example is a slight modification of our prototype example; see Example 2.2. However, let $C$ be a non-empty, convex and compact subset of $\mathbb{R}^{l}$, which intersects $\operatorname{conv}\left\{a^{1}, \ldots, a^{k}\right\}$, and define $V:=\mathbb{R}^{l}$. In what follows, we are going to investigate the following perturbed problem: given a point $\eta \in V$, find an element $x=x(\eta) \in C$ such that

$$
\left(\begin{array}{c}
\left\langle x-\left(a^{1}+\eta\right), y-x\right\rangle \\
\vdots \\
\left\langle x-a^{k}, y-x\right\rangle
\end{array}\right) \notin-\operatorname{int} \mathbb{R}_{\geq}^{k}, \quad \text { for every } \quad y \in C .
$$

In the above, the objective mapping $\mathscr{F}: V \times \mathbb{R}^{l} \rightarrow \operatorname{Mat}_{k \times l}(\mathbb{R})$ can be identified by

$$
\mathscr{F}(\eta, x):=\left(\begin{array}{c}
x-\left(a^{1}+\eta\right) \\
\vdots \\
x-a^{k}
\end{array}\right), \quad \text { for every } \quad x \in \mathbb{R}^{l} .
$$

We see at once that the assumptions of Theorem 10.1 and Theorem 10.2 are satisfied. Thus, the solution mapping $\mathrm{S}: V \rightrightarrows \mathbb{R}^{l}$ of problem (10.4) is upper semicontinuous and lower semicontinuous, and therefore continuous.

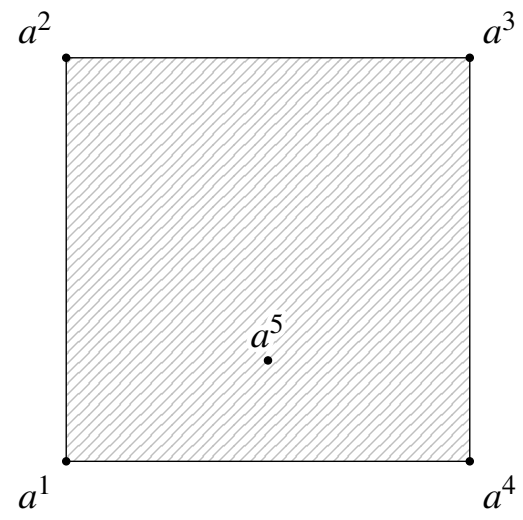

(A) $\mathrm{S}(\eta)$ for $\eta=0$.

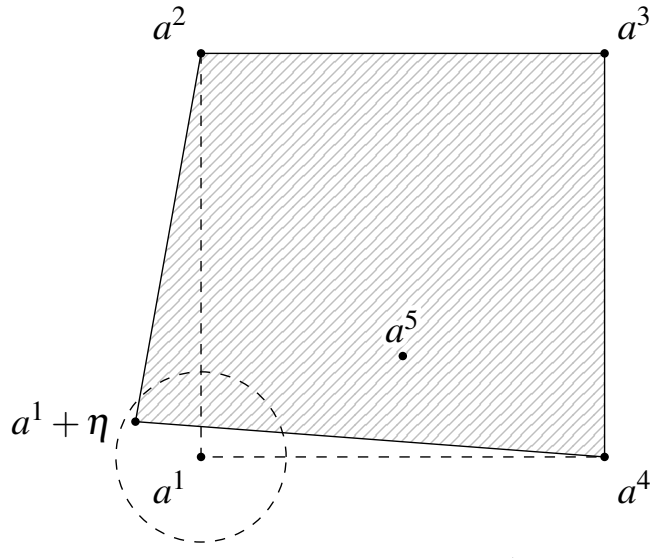

(в) $\mathrm{S}(\eta)$ for some $\eta \in U\left(a^{1}\right)$.

FIGURE 10. Illustration of the solution mapping $\mathrm{S}: V \rightrightarrows \mathbb{R}^{l}$ for the special case $l=2$ and $k=5$.

\section{FURTHER RELATED TOPICS}

11.1. Stochastic vector variational inequalities. We have seen that vector variational inequalities have several applications. However, mainly all of the research has been done in the deterministic setting without any uncertainty in the problem's data only. Recently, the authors in $[122,164,175]$ have started to extend the notion of finite-dimensional vector variational inequalities to the one of finite-dimensional stochastic (random) vector variational inequalities. To be precise, let $\mathscr{F}: \mathbb{R}^{l} \times \mathbb{R} \rightarrow$ Mat $_{k \times l}(\mathbb{R})$ be a given mapping and let $C$ be a non-empty, closed and convex subset of $\mathbb{R}^{l}$. Further, let $(\Omega, \mathscr{A}, \mathrm{P})$ be a probability space and let $\xi: \Omega \rightarrow \mathbb{R}$ be a random variable. Then, the finite-dimensional stochastic vector variational inequality consists of finding an element $x \in C$ such that almost surely

$$
\langle F(x, \xi(\omega)), y-x\rangle_{\mathbb{R}^{k}} \notin-\operatorname{int} \mathbb{R}_{\geq}^{k}, \quad \text { for every } \quad y \in C .
$$


Evidently, one can state problem (11.1) in the following way: find an element $x \in C$ such that

$$
\mathrm{P}\left(\omega \in \Omega \mid\langle F(x, \xi(\omega)), y-x\rangle_{\mathbb{R}^{k}} \notin-\operatorname{int} \mathbb{R}_{\geq}^{k} \text {, for every } y \in C\right)=1 .
$$

In general, one cannot expect that problem (11.1) has a common solution for almost every $\omega \in \Omega$. In order to get a reasonable solution in some sense, the authors in [122, 164, 175] have considered a deterministic model instead. Therefore, the authors introduce a family of weak single-valued gap functions $\left\{g_{s}(\cdot, \xi(\cdot))\right\}$ for problem (11.1); compare Remark 8.1. Under some measurability and integrability assumptions, they consider the following so-called robust weighted expected residual method (ERM) for problem (11.1):

$$
\min _{x \in C} \max _{s \in S} \mathbb{E}\left[g_{s}(x, \xi)\right]=\min _{x \in C} \max _{s \in S} \int_{\Omega} g_{s}(x, \xi(\omega)) \mathrm{P}(\mathrm{d} \omega) .
$$

In the above, one defines $S:=\left\{s \in \mathbb{R}^{k} \mid s \in \mathbb{R}_{\geq}^{k}\right.$ and $\left.\|s\|_{1}=1\right\}$ and $\mathbb{E}\left[g_{s}(x, \xi)\right]$ denotes the expected value of $g_{s}(x, \xi(\cdot)): \Omega \rightarrow \mathbb{R}$, where $x \in C$ and $s \in S$ are fixed. Zhao et al. [164] investigate the properties of a related problem, the so-called expected residual minimization problem, and derive a comprehensive convergence analysis for their approach. In [122, 175], the authors approximate problem (11.2) by a family of deterministic problems and investigate their convergence analysis. Ma et al. [122] consider a slight modification of the stochastic vector variational inequality (11.1), where they assume that the components of $\mathscr{F}: \mathbb{R}^{l} \times \Omega \rightarrow \operatorname{Mat}_{k \times l}(\mathbb{R})$ are given by $\mathscr{F}_{j}(x, \omega):=M_{j}(\omega) x+Q_{j}(\omega)$ for $x \in C$ and $\omega \in \Omega$, where $M_{j}: \Omega \rightarrow \operatorname{Mat}_{l \times l}(\mathbb{R})$ and $Q_{j}: \Omega \rightarrow \mathbb{R}^{l}$ are square integrable functions for $j=1, \ldots, k$. They show that a family of deterministic models can be used to approximate the ERM reformulation; see [122, Theorem 16]. A similar idea is used in the paper [164]. Zhao et al. further study the level sets of the objective function in ERM and discuss the convergence of stationary points; compare Section 4 in [164].

11.2. Generalized problems. Within the last years, several generalizations of the vector variational inequality (2.1) have been studied; see, for example, $[12,36,99,137]$ and the references therein. However, in what follows, we are going to consider two generalizations of problem (2.1) only: vector variational inequalities with respect to a variable domination structure and vector quasi-variational inequalities.

The first generalization is motivated by the concept of variable domination structure (or variable ordering structure, or moving domination structure), which has been introduced by Yu; see [196]. Such a concept has been frequently used in the field of multi-objective optimization [72] and has been transfered to vector variational inequalities. Recall that, given a proper, closed, convex and solid cone $K$ in $Y$, all appearing elements in problem (2.1) are compared in the following way: $x \not \mathbb{i n t}_{K} y$ if and only if $y-x \notin$ int $K$. However, from a practical point of view, it is crucial to model different preferences of a decision-maker in an adequate way. Unfortunately, ordering relations using a fixed cone are limited to describe variable preferences which is why variable orderings should be used. A main contribution, pointing out the limitations of ordering relations with respect to a fixed cone, can be found in the work by Engau; see [74]. The above limitation led to the notion of variable domination structure. Now let $\mathscr{K}: X \rightrightarrows Y$ be a mapping such that for every $x \in X$, the set $\mathscr{K}(x)$ is a proper, closed, convex and solid cone in $Y$. We say that $\mathscr{K}$ defines a variable ordering structure if elements in $Y$ are compared using $\mathscr{K}$; see [73, Definition 3.1]. Using the previous notation, the vector variational inequality with respect to the variable domination structure $\mathscr{K}$ consists of finding an element $x \in C$ such that

$$
\langle F x, y-x\rangle_{Y} \notin-\operatorname{int} \mathscr{K}(x), \quad \text { for every } y \in C .
$$

Evidently, if $\mathscr{K}$ is constant, say $\mathscr{K}(x)=K$ for every $x \in X$, where $K$ is a proper, closed, convex and solid cone in $Y$, then problem (11.3) recovers (2.1). It should be noted that most of the literature in this field is dedicated to existence results for problem (11.3). For example, some existence results, based on the Fan-KKM Lemma 5.1, can be found in [11, 31, 32, 34, 36, 63, 73, 128, 149, 163]. Here, a key 
assumption is that the set-valued mapping $Y \backslash(-\operatorname{int} \mathscr{K}(\cdot))$ is weakly closed. Note further that Elster et al. [73, Section 5.2] apply problem (11.3) to study a beam intensity optimization problem in radiotherapy treatment.

As a second prototype of generalizations of problem (2.1) we are going to consider a vector quasivariational inequality. Roughly spoken, this generalization consists of solving a set-valued fixed-point problem and a vector variational inequality at the same time. To be precise, suppose that assumption (A) holds and let a set-valued mapping $E: C \rightrightarrows C$ with non-empty, closed and convex values be given. Then, the vector quasi-variational inequality consists of finding an element $x \in C$ such that

$$
x \in E(x) \quad \text { and } \quad\langle F x, y-x\rangle_{Y} \notin-\operatorname{int} K, \quad \text { for every } y \in E(x) .
$$

Notice that in problem (11.4), the constraining set $E(x)$ depends upon the unknown element $x$. In fact, due to this dependence, the existence of solutions of problem (11.4) are challenging. In this context, the most common approach for deriving existence results is to find so-called minimal elements or equilibrium choices [67] of a certain mapping. Some existence results, which are using such a technique, can be found in $[9,45,94,109,121,131,137,142,174,185]$. Therefore, the boundedness of the underlying set $C$ is crucial. An approach, based on so-called generalized solutions, to derive existence results for problem (11.4) and related problems has recently proposed by Hebestreit et al. [19]. Note that the authors in $[65,88,115]$ use linear and non-linear scalarization techniques to derive existence results for problem (11.4). Gap functions based on scalarization schema can be found in [110, 121]. Further, approximate solutions and well-posedness conditions of problem (11.4) are discussed in [161]. Systems of vector quasi-variational inequalities have been studied in $[10,66,68]$ and applications of problem (11.4) have been proposed in $[38,115,134]$. However, if we define $Y:=\mathbb{R}$ and $K:=\mathbb{R}_{\geq}$, then problem (11.4) recovers the well-known quasi-variational inequality, which has been introduced by Bensoussan and Lions [22] in connection with a problem of impulse control. It should be noted that, within the last years, the theory of quasi-variational inequalities emerged as on of the most promising branches of pure and applied mathematics. Several applications of it can be found, for example, in economic growth models, energy production management, equilibrium problems, mechanics, physics, optimization, and numerous others; see $[17,20,62,127,147,170]$ and the references therein.

11.3. Numerical methods and algorithms. To the best of our knowledge, there are only a handful of papers, which are dealing with numerical methods or algorithms for vector variational inequalities. In $[37,51]$, the authors present a proximal-type method for problem (2.2), which is based on a linear scalarization technique. An active-set method for affine finite-dimensional vector variational inequalities has been proposed in [105, Section 4]. In [105], Goh and Yang transfer the problem to a multi-objective optimization problem and use methods from this field to derive an algorithm for the affine vector variational inequalities.

Acknowledgments. The author would like to thank Prof. Franco Giannessi and Prof. Christiane Tammer for a careful reading and numerous helpful suggestions on an earlier draft of this manuscript, which helped to improve the paper significantly.

\section{REFERENCES}

[1] R. P. Agarwal, J.-W. Chen, Y. J. Cho, Z. Wan, Stability analysis for parametric generalized vector quasivariational-like inequality problems, J. Inequal. Appl. 57 (2012), 1-15.

[2] Y. Alber, I. Ryazantseva, Nonlinear Ill-posed Problems of Monotone Type, Springer, Dordrecht, 2006.

[3] E. Allevi, A. Gnudi, I. V. Konnov, Decomposable generalized vector variational inequalities, In: K. Teo, L. Qi, X. Yang (eds), Optimization and Control with Applications. Applied Optimization, Vol. 96, pp. 497-507, Springer, Boston, MA, 2005. 
[4] H. W. Alt, Linear Functional Analysis. An Application-Oriented Introduction, Springer-Verlag, London, 2016.

[5] L. Altangerel, G. Wanka, O. Wilfer, An oriented distance function application to gap functions for vector variational inequalities, In: A. Chinchuluun, P. M. Pardalos, R. Enkhbat, E. N. Pistikopoulos (eds), Optimization, Simulation, and Control, pp. 17-34, Springer, New York, 2013.

[6] L. Q. Anh, T. Bantaojai, N. V. Hung, V. M. Tam, R. Wangkeeree, Painlevé-Kuratowski convergences of the solution sets for generalized vector quasi-equilibrium problems, Comput. Appl. Math. 37 (2018), 3832-3845.

[7] Q. H. Ansari, A note on generalized vector variational like inequalities, Optimization 41 (1997), 197-205.

[8] Q. H. Ansari, Extended generalized vector variational-like inequalities for nonmonotone multivalued maps, Ann. Sci. Math. 1 (1997), 1-11.

[9] Q. H. Ansari, W. K. Chan, X. Q. Yang, Weighted quasi-variational inequalities and constrained Nash equilibrium problems, Taiwanese J. Math. 10 (2006), 361-380.

[10] Q. H. Ansari, Y.-J. Huang, L.-J. Lin, System of vector quasi-variational inclusions with some applications, Nonlinear Anal. Theory Methods Appl. 69 (2008), 2812-2824.

[11] Q. H. Ansari, A. Khaliq, A. H. Siddiqi, On vector variational inequalities, J. Optim. Theory Appl. 84 (1995), 171-180.

[12] Q. H. Ansari, E. Köbis, J.-C. Yao, Vector Variational Inequalities and Vector Optimization, Springer, 2018.

[13] Q. H. Ansari, M. Rezaie, J. Zafarani, Generalized vector variational-like inequalities and vector optimization, J. Global Optim. 52 (2012), 271-284.

[14] Q. H. Ansari, X. Q. Yang, J.-C. Yao, Existence and duality of implicit vector variational problems, Numer. Funct. Anal. Optim. 22 (2001), 815-829.

[15] J.-P. Aubin, H. Frankowska, Set-Valued Analysis, Birkhäuser, Boston, 2009.

[16] A. Auslender, Optimisation: Méthods numérique, Masson, Paris, 1976.

[17] C. Baiocchi, A. Capelo, Variational and Quasivariational Inequalities: Applications to Free-boundary Problems, Chichester, New York, 1983.

[18] A. B. Bakushinsky, M. Y. Kokurin, A. Smirnova, Iterative Methods for Ill-posed Problems, Vol. 54, De Gruyter, Berlin, New York, 2011.

[19] T. Q. Bao, N. Hebestreit, C. Tammer, Generalized solutions of quasi-variational-like problems, submitted 2019.

[20] A. Barbagallo, Regularity results for evolutionary nonlinear variational and quasi-variational inequalities with applications to dynamic equilibrium problems, J. Global Optim. 40 (2008), 29-39.

[21] R. Benedetti, J.-J. Risler, Real Algebraic and Semi-Algebraic Sets, Hermann, Paris, 1990.

[22] A. Bensoussan, J. L. Lions, Nouvelle formulation de problemes de controle impulsionnel et applications, C. R. Acad. Sci. Paris 276 (1973), 1189-1192.

[23] C. Berge, Topological Spaces, Oliver and Boyd, Edinburgh, 1963.

[24] J. Bochnak, M. Coste, M.-F. Roy, Real Algebraic Geometry, Springer-Verlag, Berlin, Heidelberg, 1998.

[25] R. I. Boţ, S.-M. Grad, G. Wanka, Duality in Vector Optimization, Springer-Verlag, Berlin, Heidelberg, 2009.

[26] G. Bouza, C. Tammer, E. Quintana, A unified point of view to nonlinear scalarizing functionals in optimization, Vietnam J. Math. 47 (2019), 683-713.

[27] H. Brezis, Functional Analysis, Sobolev Spaces and Partial Differential Equations, Springer, New York, Dordrecht, Heidelberg, 2011.

[28] F. E. Browder, The fixed point theory of multivalued mappings in topological vector spaces, Math. Ann. 177 (1968), 283-301.

[29] C. Carathéodory, Calculus of Variations and Partial Differential Equations of the First Order. Chelsea, New York, 1982, Translation of the volume Variationsrechnung und Partielle Differential Gleichungen Erster Ordnung. B.G. Teubner, Berlin, 1935.

[30] S. Carl, V. K. Le, D. Motreanu, Nonsmooth Variational Problems and Their Inequalities. Comparison Principles and Applications, Springer US, 2007.

[31] L.-C. Ceng, S. Huang, Existence theorems for generalized vector variational inequalities with a variable ordering relation, J. Global Optim. 46 (2010), 521-535.

[32] O. Chadli, X. Q. Yang, J. C. Yao, On generalized vector pre-variational and pre-quasivariational inequalities, J. Math. Anal. Appl. 295 (2004), 392-403. 
[33] Y. Chai, X.-K. Sun, Gap functions and error bounds for generalized vector variational inequalities, Optim. Lett. 8 (2014), 1663-1673.

[34] S.-S. Chang, J. J. S. Jung, B.-S. Lee, S. J. Lee, Generalized vector version of Minty's lemma and applications, Comput. Math. Appl. 45 (2003), 647-653.

[35] C. Charitha, J. Dutta, C. S. Lalitha, Gap functions for vector variational inequalities, Optimization, 64 (2015), 1499-1520.

[36] G.-Y. Chen, Existence of solutions for a vector variational inequality: An extension of the HartmannStampacchia theorem, J. Optim. Theory Appl. 74 (1992), 445-456.

[37] Z. Chen, Asymptotic analysis for proximal-type methods in vector variational inequality problems, Oper. Res. Lett. 43 (2015), 226-230.

[38] J.-W. Chen, Y. J. Cho, Z. Wan, Nonsmooth multiobjective optimization problems and weak vector quasivariational inequalities, Comput. Appl. Math. 32 (2013), 291-301.

[39] G.-Y. Chen, C. J. Goh, X. Q. Yang, On gap functions for vector variational inequalities, In: F. Giannessi (ed), Vector Variational Inequalities and Vector Equilibria. Nonconvex Optimization and Its Applications, Vol. 38, pp. 55-72, Springer, Boston, MA, 2000.

[40] G.-Y. Chen, C. J. Goh, X. Q. Yang, Existence of a solution for generalized vector variational inequalities, Optimization 50 (2001), 1-15.

[41] G.-Y. Chen, X. Huang, X. Yang, Vector Optimization, Springer-Verlag, Berlin, Heidelberg, 2005.

[42] G.-Y. Chen, S.-H. Hou, Existence of solutions for vector variational inequalities, In: F. Giannessi (ed), Vector Variational Inequalities and Vector Equilibria. Nonconvex Optimization and Its Applications, Vol. 38, pp. 73-86, Springer, Boston, MA, 2000.

[43] J. W. Chen, E. Köbis, M. A. Köbis, J.-C. Yao, Optimality conditions for solutions of constrained inverse vector variational inequality by means of nonlinear scalarization, J. Nonlin. Var. Anal. 1 (2017), 145-158.

[44] J. Chen, E. Köbis, M. A. Köbis, J.-C. Yao, Image space analysis for constrained inverse vector variational inequalities via multiobjective optimization, J. Optim. Theory Appl. 177 (2018), 816-834.

[45] G.-Y. Chen, S. J. Li, Existence of solutions for a generalized vector quasivariational inequality, J. Optim. Theory Appl. 90 (1996), 321-334.

[46] G.-Y. Chen, S. J. Li, Properties of gap function for vector variational inequality, In: F. Giannessi, A. Maugeri (eds), Variational Analysis and Applications, Springer, New York, 2005.

[47] G.-Y. Chen, S. J. Li, Stability of weak vector variational inequality, Nonlinear Anal. 70 (2009), 1528-1535.

[48] G. Y. Chen, S. J. Li, H. Yan, Differential and sensitivity properties of gap functions for vector variational inequalities, Math. Methods Oper. Res. 57 (2003), 377-391.

[49] G. Y. Chen, S. J. Li, K. L. Teo, On the stability of generalized vector quasivariational inequality problems, J. Optim. Theory Appl. 113 (2002), 283-295.

[50] J. Chen, S. Li, Z. Wan, J.-C. Yao, Vector variational-like inequalities with constraints: Separation and alternative, J. Optim. Theory Appl. 166 (2015), 466-479.

[51] Z. Chen, L.-C. Pu, X.-Y. Wang, Generalized proximal-type methods for weak vector variational inequality problems in Banach spaces, Fixed Point Theory Appl. 191 (2015), 1-14.

[52] C.-R. Chen, H.-Z. Wei, X. Zuo, Continuity results and error bounds on pseudomonotone vector variational inequalities via scalarization, J. Funct. Spaces 3 (2016), 1-11.

[53] G.-Y. Chen, X.-Q. Yang, The vector complementary problem and its equivalences with the weak minimal element in ordered spaces, J. Math. Anal. Appl. 155 (1990), 136-158.

[54] G.-Y. Chen, X. Q. Yang, Characterizations of variable domination structures via nonlinear scalarization, J. Optim. Theory Appl. 112 (2002), 97-110.

[55] G.-Y. Chen, X. Q. Yang, H. Yu, A nonlinear scalarization function and generalized quasi-vector equilibrium problems, J. Global Optim. 32 (2005), 451-466.

[56] W. Chen, Z. Zhou, Globally proper efficiency of set-valued optimization and vector variational inequality involving the generalized contingent epiderivative, J. Inequal. Appl. 299 (2018), 1-12.

[57] Y. Cheng, On the connectedness of the solution set for the weak vector variational inequality, J. Math. Anal. Appl. 260 (2001), 1-5.

[58] Y. H. Cheng, D. L. Zhu, Global stability results for the weak vector variational inequality, J. Global Optim. 32 (2005), 543-550. 
[59] Y. Chiang, The $(S)_{+}^{1}$ conditions for generalized vector variational inequalities, J. Optim. Theory Appl. 124 (2005), 581-594.

[60] G. P. Crespi, I. Ginchev, M. Rocca, Minty vector variational inequality, efficiency and proper efficiency, Vietnam J. Math. 32 (2004), 95-107.

[61] G. P. Crespi, I. Ginchev, M. Rocca, Variational inequalities in vector optimization, In: F. Giannessi, A. Maugeri (eds), Variational Analysis and Applications. Nonconvex Optimization and Its Applications, Vol. 79, Springer, Boston, MA, 2005.

[62] P. Daniele, A remark on a dynamic model of a quasi-variational inequality, Rend. Circ. Mat. Palermo (2) Suppl. 48 (1997), 91-100.

[63] A. Daniilidis, N. Hadjisavvas, Existence theorems for vector variational inequalities, Bull. Aust. Math. Soc. 54 (1996), 473-481.

[64] L. Debnath, F.-N. Xiang, Fixed point theorems and variational inequalities with non-compact sets, Int. J. Math. Sci. 19 (1996), 111-114.

[65] X. P. Ding, The generalized vector quasi-variational-like inequalities, Comput. Math. Appl. 37 (1999), $57-67$.

[66] X. P. Ding, Systems of generalized vector quasi-variational inclusions and systems of generalized vector quasi-optimization problems in locally FC-uniform spaces, Appl. Math. Mech. 30 (2009), 263-274.

[67] X.-P. Ding, W. K. Kim, K.-K. Tan, Equilibria of generalized games with L-majorized correspondences, Int. J. Math. Math. Sci. 17 (1994), 783-790.

[68] X. P. Ding, T. C. Lai, S. J. Yu, System of generalized vector quasi-variational inclusion problems and applications to mathematical programs, Taiwanese J. Math. 13 (2009), 1515-1536.

[69] B. Djafari-Rouhani, E. Tarafdar, P. J. Watson, Fixed point theorems, coincidence theorems and variational inequalities, In: N. Hadjisavvas, J. E. Martínez-Legaz, J.-P. Penot (eds), Generalized Convexity and Generalized Monotonicity. Lecture Notes in Economics and Mathematical Systems, Vol. 502, Springer, Berlin, Heidelberg, 2001.

[70] M. Ehrgott, Multicriteria Optimization, Springer, Berlin, Heidelberg, 2005.

[71] M. Ehrgott, A. Raith, On vector equilibria, vector optimization and vector variational inequalities, J. MultiCrit. Decis. Anal. 18 (2011), 39-54.

[72] G. Eichfelder, Variable Ordering Structures in Vector Optimization, Springer-Verlag, Berlin, Heidelberg, New York, 2014.

[73] R. Elster, N. Hebestreit, A. A. Khan, C. Tammer, Inverse generalized vector variational inequalities with respect to variable domination structures and applications to vector approximation problems, Appl. Anal. Optim. 2 (2018), 341-372.

[74] A. Engau, Variable preference modeling with ideal-symmetric convex cones, J. Glob. Optim. 42 (2008), 295-311.

[75] F. Facchinei, J.-S. Pang, Finite-Dimensional Variational Inequalities and Complementary Problems, Vol. 1, Springer, New York, Berlin, Heidelberg, 2000.

[76] K. Fan, A generalization of Tychonoff's fixed point theorem, Math. Ann. 142 (1961), 305-310.

[77] K. Fan, A minimax inequality and application, In: O. Shisha (ed), Inequalities III, pp. 103-113, Academic Press, San Diego, 1972.

[78] J.-H. Fan, Y.-L. Wang, R.-Y. Zhong, Connectedness of solution sets for weak vector variational inequalities on unbounded closed convex sets, Abstr. Appl. Anal. 2013 (2013), 1-5.

[79] Y.-P. Fang, R. Hu, On the nonemptiness and compactness of the solution sets for vector variational inequalities, Optimization 59 (2010), 1107-1116.

[80] Y.-P. Fang, N.-J. Huang, Existence results for systems of strong implicit vector variational inequalities, Acta Math. Hungar. 103 (2004), 265-279.

[81] Y.-P. Fang, N.-J. Huang, On vector variational inequalities in reflexive Banach spaces, J. Global Optim. 32 (2005), 495-505.

[82] Y.-P. Fang, N.-J. Huang, Strong vector variational inequalities in Banach spaces, Appl. Math. Lett. 19 (2006), 362-368.

[83] Y.-P. Fang, N.-J. Huang, Well-posedness for vector variational inequality and constrained vector optimization, Taiwanese J. Math. 11 (2007), 1287-1300. 
[84] Y. P. Fang, X. X. Huang, X. Q. Yang, Characterizing the nonemptiness and compactness of the solution set of a vector variational inequality by scalarization, J. Optim. Theory Appl. 162 (2014), 548-558.

[85] Z. M. Fang, S. J. Li, Upper semicontinuity of solution maps for a parametric weak vector variational inequality, J. Inequal. Appl. 2010 (2010), 1-6.

[86] Z. Fang, M. Li, S. Li, Stability and sensitivity analysis of solutions to weak vector variational inequalities, Set-Valued Var. Anal. 20 (2012), 111-129.

[87] Z. M. Fang, S. J. Li, K. L. Teo, Painleve-Kuratowski convergences for the solution sets of set-valued weak vector variational inequalities, J. Inequal. Appl. 2008 (2008), 1-14.

[88] A. Farajzadeh, B. S. Lee, S. Plubteing, On generalized quasi-vector equilibrium problems via scalarization method, J. Optim. Theory Appl. 168 (2016), 584-599.

[89] A. Farajzadeh, S. Plubteing, On parametric implicit vector variational inequality problems, J. Inequal. Appl. 2014 (2014), 151-158.

[90] D. Farenick, Fundamentals of Functional Analysis, Springer International Publishing, Switzerland, 2016.

[91] G. Fichera, Sul problema elastostatico di Signorini con ambigue condizioni al contorno (On Signorini's elastostatic problem with ambiguous boundary conditions), Atti. Acad. naz. Lincei, Cl. Sci. fis. mat. natur. 8 (1963), 138-142.

[92] J. Fu, Vector optimization for a class of nonconvex functions, Indian J. Pure Appl. Math. 31 (2000), 15371543.

[93] W.-T. Fu, S.-Y. Wang, W.-S. Xiao, M. Yu, On the existence and connectedness of solution sets of vector variational inequalities, Math. Methods Oper. Res. 54 (2001), 201-215.

[94] X. Gang, S. Liu, Existence of solutions for generalized vector quasi-variational-like inequalities without monotonicity, Comput. Math. Appl. 58 (2009), 1550-1557.

[95] D. Y. Gao, Canonical Duality Theory, Springer, New York, 2017.

[96] F. Giannessi, Theorem of the alternative, quadratic programs, and complementary problems, In: R. W. Cottle, F. Gianessi, J.-L. Lions (eds), Variational Inequalities and Complementarity Problems, John Wiley and Sons, Chichester, 1980.

[97] F. Giannessi, Embedding variational inequalities and their generalizations into a separation scheme, J. Inequal. Appl. 1 (1997), 139-147.

[98] F. Giannessi, On Minty variational principle, In: F. Giannessi, S. Komlósi, T. Rapcsák (eds), New Trends in Mathematical Programming. Applied Optimization, Vol. 13, pp. 93-99, Springer, Boston, MA, 1998.

[99] F. Giannessi (ed), Vector Variational Inequalities and Vector Equilibria, Kluwer Academic Publishers, Dordrecht, 2000.

[100] F. Giannessi, Constrained Optimization and Image Space Analysis, Separation of Sets and Optimality Conditions, Springer, Berlin, 2005.

[101] F. Giannessi, G. Mastroeni, On the theory of vector optimization and variational inequalities. Image space analysis and separation, In: F. Giannessi (ed), Vector Variational Inequalities and Vector Equilibria. Nonconvex Optimization and Its Applications, Vol. 38, pp. 153-215, Springer, Boston, MA, 2000.

[102] F. Giannessi, G. Mastroeni, X. Q. Yang, Survey on vector complementarity problems, J. Global Optim. 53(1) (2012), 53-67.

[103] F. Giannessi, A. Maugeri, Variational Analysis and Applications, Springer, New York, 2005.

[104] F. Giannessi, L. Pellegrini, Image space analysis for vector optimization and variational inequalities. Scalarization, In: P. M. Pardalos, A. Migdalas, R. E. Burkard (eds), Combinatorial and Global Optimization, Vol. 14, pp. 97-110, World Scientific, 2002.

[105] C.-J. Goh, X. Q. Yang, Scalarization methods for vector variational inequality, In: F. Giannessi (ed), Vector Variational Inequalities and Vector Equilibria. Nonconvex Optimization and Its Applications, Vol. 38, pp. 153-215, Springer, Boston, MA, 2000.

[106] C.-J. Goh, X. Q. Yang, Duality in Optimization and Variational Inequalities, Taylor and Francis, New York, 2002.

[107] X. Gong, W. Liu, Proper efficiency for set-valued vector optimization problems and vector variational inequalities, Math. Methods Oper. Res. 51 (2000), 443-457.

[108] A. Göpfert, C. Tammer, C. Zălinescu, Variational Methods in Partially Ordered Spaces, Springer-Verlag, New York, 2003. 
[109] S. Gupta, S. Husain, Existence of solutions for generalized nonlinear vector quasi-variational-like inequalities with set-valued mappings, Filomat 26 (2012), 909-916.

[110] R. Gupta, A. Mehra, Gap functions and error bounds for set-valued vector quasi variational inequality problems, Appl. Math. 8 (2017), 1903-1917.

[111] S.-M. Guu, N.-J. Huang, J. Li, Scalarization approaches for set-valued vector optimization problems and vector variational inequalities, J. Math. Anal. Appl. 356 (2009), 564-676.

[112] N. Hadjisavvas, S. Schaible, From scalar to vector equilibrium problems in the quasimonotone case, J. Optim. Theory Appl. 96 (1998), 297-309.

[113] P. T. Harker, J.-S. Pang, Finite-dimensional variational inequality and nonlinear complementary problems: A survey of theory, algorithms and applications, Math. Program. 48 (1990), 161-220.

[114] Z.-Q. He, J. Li, Gap functions and existence of solutions to generalized vector variational inequalities, J. Global Optim. 34 (2006), 427-440.

[115] N. Hebestreit, A. A. Khan, E. Köbis, C. Tammer, Existence theorems and regularization methods for noncoercive vector variational and vector quasi-variational inequalities, J. Nonlinear Convex Anal. 20 (2019), $565-591$.

[116] J.-B. Hiriart-Urruty, New concepts in nondifferentiable programming, Mémoires de la Société Mathématique de France 60 (1979), 57-85.

[117] J.-B. Hiriart-Urruty, Tangent cones, generalized gradients and mathematical programming in Banach spaces, Math. Oper. Res. 4 (1979), 79-97.

[118] J. B. Hiriart-Urruty, Images of connected sets by semicontinuous multifunctions, J. Math. Anal. Appl. 111 (1985), 407-422.

[119] V. T. Hieu, Disconnectedness and unboundedness of the solution sets of monotone vector variational inequalities, Appl. Anal. (2019), 1-12.

[120] V. T. Hieu, Numbers of the connected components of the solution sets of monotone affine vector variational inequalities, J. Global Optim. 73 (2019), 223-237.

[121] N.-J. Huang, X.-J. Long, Gap functions and existence of solutions for generalized vector quasivariational inequalities, Involve 183 (2008), 183-195.

[122] N.-Q. Huang, H.-Q. Ma, D. O'Regan, M. Wu, A new gap function for vector variational inequalities with an application, J. Appl. Math. (2013), 1-8.

[123] N. Huang, F. Yaping, System of generalized vector variational inequalities, Acta Math. Sci. Ser. A Chin. Ed. 25 (2005), 587-593.

[124] N.-J. Huang, R.-Y. Zhong, Lower semicontinuity for parametric weak vector variational inequalities in reflexive Banach spaces, J. Optim. Theory Appl. 150 (2011), 317-326.

[125] N. T. T. Huong, J.-C. Yao, N. D. Yen, Connectedness structure of the solution sets of vector variational inequalities, Optimization 66 (2017), 889-901.

[126] J. Jahn, Vector Optimization, Springer-Verlag, Berlin, Heidelberg, 2011.

[127] A. Kadoya, N. Kenmochi, M. Niezgódka, Quasi-variational inequalities in economic growth models with technological development, Adv. Math. Sci. Appl. 24 (2014), 185-214.

[128] S. A. Khan, F. Suhel, Existence of solutions of mixed vector variational-like inequalities, Bull. Math. Anal. Appl. 3 (2011), 102-109.

[129] A. A. Khan, C. Tammer, C. Zălinescu, Set-valued Optimization, Springer-Verlag, Berlin, Heidelberg, 2015.

[130] S. A. Khan, F. Usman, A generalized mixed vector variational-like inequality problem, Nonlinear Anal. 71 (2009), 5354-5362.

[131] A. Khaliq, M. Rashid, On generalized vector quasivariational-like inequality problems, Fixed Point Theory Appl. 3 (2005), 243-255.

[132] M. F. Khan, B. S. Lee, Salahuddin, Vector F-implicit complementary problems with corresponding variational inequality problems, Appl. Math. Lett. 20 (2007), 433-438.

[133] M. F. Khan, Salahuddin, On generalized vector variational-like inequalities, Nonlinear Anal. Theory Methods Appl. 59 (2004), 879-889.

[134] P. Q. Khanh, L. M. Luu, On the existence of solutions to vector quasivariational inequalities and quasicomplementary problems with applications to traffic network equilibria, J. Optim. Theory Appl. 123 (2004), 533-548. 
[135] D. S. Kim, H. Kuk, G. M. Lee, Existence of solutions for vector optimization problems, J. Math. Anal. Appl. 220 (1998), 90-98.

[136] M. H. Kim, S. H. Kum, G. M. Lee, Vector variational inequalities involving vector maximal points, J. Optim. Theory Appl. 114 (2002), 593-607.

[137] W. K. Kim, S. Kum, An extension of generalized vector quasi-variational inequality, Commun. Korean. Math. Soc. 21 (2006), 273-285.

[138] D. S. Kim, G. M. Lee, B. S. Lee, G.-Y. Chen, Generalized vector variational inequality and its duality for set-valued maps, Appl. Math. Lett. 4 (1998), 21-26.

[139] D. S. Kim, G. M. Lee, B. S. Lee, S. J. Cho, Generalized vector variational inequality and fuzzy extension, Appl. Math. Lett. 6 (1993), 47-51.

[140] D. S. Kim, G. M. Lee, B. S. Lee, S. J. Cho, On vector variational inequality, Bull. Korean Math. Soc. 33 (1996), 553-563.

[141] D. S. Kim, G. M. Lee, B. S. Lee, N. D. Yen, Vector variational inequality as a tool for studying vector optimization, Nonlinear Anal. 34 (1998), 745-765.

[142] W. K. Kim, K.-K. Tan, On generalized vector quasi-variational inequalities, Optimization 46 (1999), 185198.

[143] K. Kimura, Y. C. Liou, S.-Y. Wu, J.-C. Yao, Well-posedness for parametric vector equilibrium problems with applications, J. Ind. Manag. Optim. 4 (2008), 313-327.

[144] D. Kinderlehrer, G. Stampacchia, An Introduction to Variational Inequalities and their Applications, Academic Press, New York, London, 1980.

[145] S. Komlósi, On the Stampacchia and Minty variational inequalities, In: G. Giorgi, F. A. Rossi (eds), Generalized Convexity and Optimization for Economic and Financial Decisions, Pitagora Editrice, 1999.

[146] I. V. Konnov, J.-C. Yao, On the generalized vector variational inequality problem, J. Math. Anal. Appl. 206 (1997), 42-58.

[147] A. S. Kravchuk, P. J. Neittaanmäki, Variational and Quasi-Variational Inequalities in Mechanics, Springer, Dordrecht, 2007.

[148] E. Kreyszig, Introductory Functional Analysis with Applications, John Wiley, New York, 1978.

[149] S. Kum, G. M. Lee, J.-C. Yao, An existence result for implicit vector variational inequality with multifunctions, Appl. Math. Lett. 16 (2003), 453-458.

[150] V. Laha, S. K. Mishra, On $V$-r-invexity and vector variational-like inequalities, Filomat 26 (2012), 10651073.

[151] T. C. Lai, J.-C. Yao, Existence results for VVIP, Appl. Math. Lett. 3 (1996), 17-19.

[152] M. Lassonde, On the use of KKM multifunctions in fixed point theory and related topics, J. Math. Anal. Appl. 97 (1983), 151-201.

[153] G. M. Lee, K. B. Lee, Vector variational inequalities for nondifferentiable convex vector optimization problems, J. Global. Optim. 32 (2005), 597-612.

[154] G. M. Lee, D. E. Ward, On relations between vector optimization problems and vector variational inequalities, J. Optim. Theory Appl. 113 (2002), 583-596.

[155] G. M. Lee, N. D. Yen, On monotone and strongly monotone vector variational inequalities, In: F. Giannessi (ed), Vector Variational Inequalities and Vector Equilibria. Nonconvex Optimization and Its Applications, Vol. 38, pp. 467-478, Springer, Boston, MA, 2000.

[156] G. M. Lee, N. D. Yen, A result on vector variational inequalities with polyhedral constraint sets, J. Optim. Theory Appl. 109 (2001), 193-197.

[157] M. Li, Error bounds of regularized gap functions for weak vector variational inequality problems, J. Inequal. Appl. 331 (2014), 1-7.

[158] J. Li, N. J. Huang, Image space analysis for vector variational inequalities with matrix inequality constraints and applications, J. Optim. Theory Appl. 145 (2010), 459-477.

[159] J. Li, G. Mastroeni, Vector variational inequalities involving set-valued mappings via scalarization with applications to error bounds for gap function, J. Optim. Theory Appl. 145 (2010), 355-372.

[160] S. J. Li, Y. D. Xu, Gap functions and error bounds for weak vector variational inequalities, Optimization 63 (2014), 1339-1352. 
[161] M. B. Lignola, J. Morgan, Vector quasi-variational inequalities: Approximate solutions and well-posedness, J. Convex Anal. 13 (2006), 373-384.

[162] M. B. Lignola, J. Morgan, V. Scalzo, Lower convergence of approximate solutions to vector quasivariational problems, Optimization 59 (2010), 821-832.

[163] K. L. Lin, D. P. Yang, J.-C. Yao, Generalized vector variational inequalities, J. Optim. Theory Appl. 92 (1997), 117-125.

[164] G.-H. Lin, X. Yang, J. Zhang, Y. Zhao, Expected residual minimization formulation for a class of stochastic vector variational inequalities, J. Optim. Theory Appl. 175 (2017), 545-566.

[165] L.-J. Lin, Pre-vector variational inequalities, Bull. Austral. Math. Soc. 53 (1996), 63-70.

[166] Q. Luo, Ky Fan's section theorem and its applications in topological ordered spaces, Appl. Math. Lett. 17 (2004), 1113-1119.

[167] D. X. Luong, Penalty functions for the vector variational inequality problem, Acta Math. Vietnam, 37 (2012), 31-40.

[168] G. Mastreoni, On the image space analysis for vector quasi-equilibrium problems with a variable ordering relation, J. Global Optim. 53 (2012), 203-214.

[169] U. Mosco, Dual variational inequalities, J. Math. Anal. Appl. 40 (1972), 202-206.

[170] K. Naito, A system of quasivariational inequalities and its application to reaction diffusion equations, Publ. Res. Inst. math. Sci. 21 (1985), 677-698.

[171] Z. Naniewicz, P. D. Panagiotopoulos. Mathematical Theory of Hemivariational Inequalities and Applications, Marcel Dekker, New York, Basel, Honk Kong, 1995.

[172] R. Osuna-Gómez, A. Rufián-Lizana, G. Ruiz-Garzón, Relations between vector variational-like inequality and optimization problems, Eur. J. Oper. Res. 157 (2004), 113-119.

[173] S. Park, Recent applications of the Fan-KKM theorem, J. Nonlinear Convex Anal. (2013), 58-68.

[174] J.-W. Peng, X.-M. Yang, Generalized vector quasi-variational-like inequalities, J. Global Optim. 53 (2012), 271-284.

[175] Z. Y. Peng, Y. Zhao, Y. B. Zhao, Robust weighted expected residual minimization formulation for stochastic vector variational inequalities, J. Nonlinear Sci. Appl. 10 (2017), 5825-5833.

[176] S. Plubtieng, T. Thammathiwat, Existence of solutions of new generalized mixed vector variational-like inequalities in reflexive Banach spaces, J. Optim. Theory Appl. 162 (2014), 589-604.

[177] N. Popovici, M. Rocca, Pareto reducibility of vector variational inequalities, Economics and Quantitative Methods, Department of Economics, University of Insubria, 2010.

[178] N. Popovici, Pareto reducible multicriteria optimization problems, Optimization 54 (2005), 253-263.

[179] R. T. Rockafellar, Convex Analysis, Princeton University Press, Princeton, New Jersey, 1970.

[180] W. Rudin, Functional Analysis, McGraw-Hill, New York, 1973.

[181] G. Stampacchia, Formes bilinéaires coercitives sur les ensembles convexes, Académie des Sciences de Paris, 258 (1964), 4413-4416.

[182] K. L. Teo, X. M. Yang, X. Q. Yang, Some remarks on the Minty vector variational inequality, J. Optim. Theory Appl. 121 (2004), 193-201.

[183] A. N. Tykhonov, On the stability of the functional optimization problem, USSR J. Comput. Math. Math. Physics, 6 (1966), 631-634.

[184] S. Waldmann, Topology. An Introduction, Springer, Heidelberg, New York, 2014.

[185] R. Wangkeeree, P. Yimmuang, Generalized nonlinear vector mixed quasi-variational-like inequality governed by a multi-valued map, J. Inequal. Appl. 294 (2013), 1-11.

[186] Z. Xia, Y. Zhao, On the existence of solutions of vector variational like inequalities, Nonlinear Anal. 64 (2006), 2075-2083.

[187] Y. D. Xu, Nonlinear separation approach to inverse variational inequalities, Optimization 65 (2016), 13151335.

[188] X. Q. Yang, Generalized convex functions and vector variational inequalities, J. Optim. Theory Appl. 79 (1993), 563-580.

[189] X. Q. Yang, Vector variational inequalities and its duality, Nonlinear Anal. 22 (1993), 869-877.

[190] X. Q. Yang, Vector variational inequality and vector pseudolinear optimization, J. Optim. Theory Appl. 95 (1997), 729-734. 
[191] X. Q. Yang, G.-Y. Chen, On inverse vector variational inequalities, In: F. Giannessi (ed), Vector Variational Inequalities and Vector Equilibria. Nonconvex Optimization and Its Applications, Vol. 38, pp. 433-446, Springer, Boston, MA, 2000.

[192] X. M. Yang, X. Q. Yang, Vector variational-like inequality with pseudoinvexity, Optimization 55 (2006), 157-170.

[193] J.-C. Yao, S. J. Yu, On vector variational inequalities, J. Optim. Theory Appl. 89 (1996), 749-769.

[194] J.-C. Yao, L.-C. Zeng, Existence of solutions of generalized vector variational inequalities in reflexive Banach spaces, J. Global Optim. 36 (2006), 483-497.

[195] N. D. Yen, An introduction to vector variational inequalities and some new results, Acta Math Vietnam 41 (2016), 505-529.

[196] P. L. Yu, Cone convexity, cone extreme points, and nondominated solutions in decision problems with multiobjectives, J. Optim. Theory Appl. 14 (1974), 319-377.

[197] E. Zeidler, Nonlinear Functional Analysis and its Applications I, Springer-Verlag, New York, Berlin, Heidelberg, 1986.

[198] E. Zeidler, Nonlinear Functional Analysis and its Application IIA, Springer-Verlag, New York, Berlin, Heidelberg, 1990.

[199] E. Zeidler, Nonlinear Functional Analysis and its Application IIB, Springer-Verlag, New York, Berlin, Heidelberg, 1990.

[200] References on vector variational inequalities, J. Global Optim. 32 (2005), 529-536. 